\title{
WELL-POSEDNESS OF THREE-DIMENSIONAL ISENTROPIC COMPRESSIBLE NAVIER-STOKES EQUATIONS WITH DEGENERATE VISCOSITIES AND FAR FIELD VACUUM
}

\author{
ZHOUPING XIN AND SHENGGUO ZHU
}

\begin{abstract}
In this paper, the Cauchy problem for the three-dimensional (3-D) isentropic compressible Navier-Stokes equations is considered. When viscosity coefficients are given as a constant multiple of the density's power $\left(\rho^{\delta}\right.$ with $0<\delta<1$ ), based on some analysis of the nonlinear structure of this system, we identify the class of initial data admitting a local regular solution with far field vacuum and finite energy in some inhomogeneous Sobolev spaces by introducing some new variables and initial compatibility conditions, which solves an open problem of degenerate viscous flow partially mentioned by BreshDesjardins-Metivier [3], Jiu-Wang-Xin [1] and so on. Moreover, in contrast to the classical theory in the case of the constant viscosity, we show that one can not obtain any global regular solution whose $L^{\infty}$ norm of $u$ decays to zero as time $t$ goes to infinity.
\end{abstract}

\section{INTRODUCTION}

The time evolution of the mass density $\rho \geq 0$ and the velocity $u=\left(u^{(1)}, u^{(2)}, u^{(3)}\right)^{\top} \in \mathbb{R}^{3}$ of a general viscous isentropic compressible fluid occupying a spatial domain $\Omega \subset \mathbb{R}^{3}$ is governed by the following isentropic compressible Navier-Stokes equations (ICNS):

$$
\left\{\begin{array}{l}
\rho_{t}+\operatorname{div}(\rho u)=0, \\
(\rho u)_{t}+\operatorname{div}(\rho u \otimes u)+\nabla P=\operatorname{div} \mathbb{T} .
\end{array}\right.
$$

Here, $x=\left(x_{1}, x_{2}, x_{3}\right) \in \Omega, t \geq 0$ are the space and time variables, respectively. For the polytropic gases, the constitutive relation is given by

$$
P=A \rho^{\gamma}, \quad A>0, \quad \gamma>1,
$$

where $A$ is an entropy constant and $\gamma$ is the adiabatic exponent. $\mathbb{T}$ denotes the viscous stress tensor with the form

$$
\mathbb{T}=\mu(\rho)\left(\nabla u+(\nabla u)^{\top}\right)+\lambda(\rho) \operatorname{div} u \mathbb{I}_{3},
$$

where $\mathbb{I}_{3}$ is the $3 \times 3$ identity matrix,

$$
\mu(\rho)=\alpha \rho^{\delta}, \quad \lambda(\rho)=\beta \rho^{\delta},
$$

for some constant $\delta \geq 0, \mu(\rho)$ is the shear viscosity coefficient, $\lambda(\rho)+\frac{2}{3} \mu(\rho)$ is the bulk viscosity coefficient, $\alpha$ and $\beta$ are both constants satisfying

$$
\alpha>0, \quad \text { and } 2 \alpha+3 \beta \geq 0 \text {. }
$$

Date: Oct. 31th, 2018.

2010 Mathematics Subject Classification. Primary: 35A01, 35B40, 76N10; Secondary: 35B65, 35A09.

Key words and phrases. Compressible Navier-Stokes equations, Three dimensions, Far field vacuum, Degenerate viscosity, Classical solutions, Local-in-time well-posedness, Asymptotic behavior. 
Let $\Omega=\mathbb{R}^{3}$. Assuming $0<\delta<1$, we look for a smooth solution $(\rho, u)$ with finite energy to the Cauchy problem for (1.1)-(1.5) with the following initial data and far field behavior:

$$
\begin{array}{ll}
\left.(\rho, u)\right|_{t=0}=\left(\rho_{0}(x) \geq 0, u_{0}(x)\right) & \text { for } \quad x \in \mathbb{R}^{3}, \\
(\rho, u)(t, x) \rightarrow(0,0) \quad \text { as } \quad|x| \rightarrow \infty & \text { for } t \geq 0 .
\end{array}
$$

In the theory of gas dynamics, the CNS can be derived from the Boltzmann equations through the Chapman-Enskog expansion, cf. Chapman-Cowling [4] and Li-Qin [16]. Under some proper physical assumptions, the viscosity coefficients and heat conductivity coefficient $\kappa$ are not constants but functions of the absolute temperature $\theta$ such as:

$$
\mu(\theta)=a_{1} \theta^{\frac{1}{2}} F(\theta), \quad \lambda(\theta)=a_{2} \theta^{\frac{1}{2}} F(\theta), \quad \kappa(\theta)=a_{3} \theta^{\frac{1}{2}} F(\theta)
$$

for some constants $a_{i}(i=1,2,3)$ (see [4]). Actually for the cut-off inverse power force models, if the intermolecular potential varies as $r^{-a}$, where $r$ is intermolecular distance, then in (1.8):

$$
F(\theta)=\theta^{b} \quad \text { with } \quad b=\frac{2}{a} \in[0,+\infty) .
$$

In particular, for Maxwellian molecules, $a=4$ and $b=\frac{1}{2}$; for rigid elastic spherical molecules, $a=\infty$ and $b=0$; while for ionized gas, $a=1$ and $b=2$ (see $\S 10$ of [4]).

According to Liu-Xin-Yang [20], for isentropic and polytropic fluids, such a dependence is inherited through the laws of Boyle and Gay-Lussac:

$$
P=R \rho \theta=A \rho^{\gamma}, \quad \text { for } \text { constant } R>0,
$$

i.e., $\theta=A R^{-1} \rho^{\gamma-1}$, and one finds that the viscosity coefficients are functions of the density of the form (1.4) with $0<\delta<1$ in many cases.

Throughout this paper, we adopt the following simplified notations, most of them are for the standard homogeneous and inhomogeneous Sobolev spaces:

$$
\begin{aligned}
& \|f\|_{s}=\|f\|_{H^{s}\left(\mathbb{R}^{3}\right)}, \quad|f|_{p}=\|f\|_{L^{p}\left(\mathbb{R}^{3}\right)}, \quad\|f\|_{m, p}=\|f\|_{W^{m, p}\left(\mathbb{R}^{3}\right)}, \quad|f|_{C^{k}}=\|f\|_{C^{k}\left(\mathbb{R}^{3}\right)}, \\
& \|f\|_{X Y(t)}=\|f\|_{X\left([0, t] ; Y\left(\mathbb{R}^{3}\right)\right)}, \quad \dot{H}^{\iota}=\left\{f:\left.\mathbb{R}^{3} \rightarrow \mathbb{R}\left|\|f\|_{\dot{H}^{\iota}}^{2}=\int\right| \xi\right|^{2 \iota}|\hat{f}(\xi)|^{2} \mathrm{~d} \xi<\infty\right\}, \\
& D^{k, r}=\left\{f \in L_{l o c}^{1}\left(\mathbb{R}^{3}\right):|f|_{D^{k, r}}=\left|\nabla^{k} f\right|_{r}<+\infty\right\}, \quad D^{k}=D^{k, 2}, \\
& D^{1}=\left\{f \in L^{6}\left(\mathbb{R}^{3}\right):|f|_{D^{1}}=|\nabla f|_{2}<\infty\right\}, \quad|f|_{D^{1}}=\|f\|_{D^{1}\left(\mathbb{R}^{3}\right)}, \\
& \|f\|_{X_{1} \cap X_{2}}=\|f\|_{X_{1}}+\|f\|_{X_{2}}, \quad \int_{\mathbb{R}^{3}} f \mathrm{~d} x=\int f, \quad X\left([0, T] ; Y\left(\mathbb{R}^{3}\right)\right)=X([0, T] ; Y) .
\end{aligned}
$$

A detailed study of homogeneous Sobolev spaces can be found in [8].

When $\inf _{x} \rho_{0}(x)>0$, the local well-posedness of classical solutions for (1.1)-(1.7) follows from the standard symmetric hyperbolic-parabolic structure which satisfies the well-known Kawashima's condition, c.f. [12, 24]. However, such an approach fails in the presence of the vacuum due to the degeneracies of the time evolution and viscosities. Generally vacuum will appear in the far field under some physical requirements such as finite total mass in $\mathbb{R}^{3}$. One of the main issues in the presence of vacuum is to understand the behavior of the velocity field near the vacuum. For the constant viscosity flow $(\delta=0$ in (1.4)), a 
remedy was suggested by Cho-Choe-Kim [5], where they imposed initially a compatibility condition:

$$
-\operatorname{div} \mathbb{T}_{0}+\nabla P\left(\rho_{0}\right)=\sqrt{\rho_{0}} g, \quad \text { for some } g \in L^{2}\left(\mathbb{R}^{3}\right),
$$

which leads to

$$
\left(\sqrt{\rho} u_{t}, \nabla u_{t}\right) \in L^{\infty}\left(\left[0, T_{*}\right] ; L^{2}\right)
$$

for a short time $T_{*}>0$. Then they established successfully the local well-posedness of smooth solutions with vacuum in some Sobolev spaces of $\mathbb{R}^{3}$, and also showed that this kind of initial compatibility condition is necessary for their solution class.

For density-dependent viscosities $(\delta>0$ in (1.4)), the strong degeneracy of the momentum equations in (1.1) near the vacuum creates serious difficulties for the well-posedness of both strong and weak solutions. Though some significant achievements [2, 3, 7, 9, 15, 17, 20, 23, 29, 31, 32 have been obtained, however, a lot of fundamental questions remain open, including the local well-posedness of classical solutions with finite energy in multidimensions for $\delta \in(0,1)$. Our result obtained in this paper has taken a first step toward this direction.

Compared with flows of constant viscosities, the analysis of the degeneracies in the momentum equations $(1.1)_{2}$ requires some special attentions. Indeed, for $\delta=0$ in [5], the uniform ellipticity of the Lamé operator $L$ defined by

$$
L u \triangleq-\alpha \Delta u-(\alpha+\beta) \nabla \operatorname{div} u
$$

plays an essential role in the regularity estimates on $u$. One can use the standard elliptic theory to estimate $|u|_{D^{k+2}}$ by the $D^{k}$-norm of all other terms in the momentum equations. However, for $\delta>0$, the viscosity coefficients vanish in the presence of vacuum, which makes it difficult to adapt the approach in [5] to the current case.

For the cases $\delta \in(0, \infty)$, if $\rho>0,(1.1)_{2}$ can be formally rewritten as

$$
u_{t}+u \cdot \nabla u+\frac{A \gamma}{\gamma-1} \nabla \rho^{\gamma-1}+\rho^{\delta-1} L u=\psi \cdot Q(u)
$$

where the quantities $\psi$ and $Q(u)$ are given by

$$
\begin{aligned}
\psi & \triangleq \nabla \log \rho \quad \text { when } \quad \delta=1 ; \\
\psi & \triangleq \frac{\delta}{\delta-1} \nabla \rho^{\delta-1} \quad \text { when } \quad \delta \in(0,1) \cup(1, \infty) ; \\
Q(u) & \triangleq \alpha\left(\nabla u+(\nabla u)^{\top}\right)+\beta \operatorname{div} u \mathbb{I}_{3} .
\end{aligned}
$$

When $\delta=1$, according to (1.9)-(1.10), the degeneracies of the time evolution and viscosities on $u$ caused by the vacuum have been transferred to the possible singularity of the term $\nabla \log \rho$, which actually can be controlled by a symmetric hyperbolic system with a source term $\nabla$ div $u$ in Li-Pan-Zhu [18]. Then via establishing a uniform a priori estimates in $L^{6} \cap D^{1} \cap D^{2}$ for $\nabla \log \rho$, the existence of 2-D local classical solution with far field vacuum to (1.1) has been obtained in [18], which also applies to the 2-D shallow water equations. When $\delta>1$, (1.9)-(1.10) imply that actually the velocity $u$ can be governed by a nonlinear degenerate parabolic system without singularity near the vacuum region. Based on this observation, by using some hyperbolic approach which bridges the parabolic system (1.9) when $\rho>0$ and the hyperbolic one $u_{t}+u \cdot \nabla u=0$ when $\rho=0$, the existence of 3-D local classical solutions with vacuum to (1.1) was established in Li-Pan-Zhu [19]. 
The corresponding global well-posedness in some homogeneous Sobolev spaces has been established by Xin-Zhu [29] under some initial smallness assumptions.

However, such approaches used in [18, 19, 29] fail to apply to the case $\delta \in(0,1)$. Indeed, when vacuum appears only at far fields, the velocity field $u$ is still governed by the quasilinear parabolic system (1.9)-(1.10). Yet, some new essential difficulties arise compared with the case $\delta \geq 1$ :

(1) first, the source term contains a stronger singularity as:

$$
\nabla \rho^{\delta-1}=(\delta-1) \rho^{\delta-1} \nabla \log \rho,
$$

whose behavior will become more singular than that of $\nabla \log \rho$ in [18] due to $\delta-1<0$ when the density $\rho \rightarrow 0$;

(2) second, the coefficient $\rho^{\delta-1}$ in front of the Lamé operator $L$ will tend to $\infty$ as $\rho \rightarrow 0$ in the far filed instead of equaling to 1 in [18] or tending to 0 in [19, 29] Then it is necessary to show that the term $\rho^{\delta-1} L u$ is well defined.

Therefore, the three quantities

$$
\left(\rho^{\gamma-1}, \nabla \rho^{\delta-1}, \rho^{\delta-1} L u\right)
$$

will play significant roles in our analysis on the higher order regularities of the fluid velocity $u$. Due to this observation, we first introduce a proper class of solutions called regular solutions to the Cauchy problem (1.1)-(1.7).

Definition 1.1. Let $T>0$ be a finite constant. A solution $(\rho, u)$ to the Cauchy problem (1.1)-(1.7) is called a regular solution in $[0, T] \times \mathbb{R}^{3}$ if $(\rho, u)$ satisfies this problem in the sense of distribution and:

$$
\begin{aligned}
\text { (A) } & \rho>0, \quad \rho^{\gamma-1} \in C\left([0, T] ; H^{3}\right), \quad \nabla \rho^{\delta-1} \in L^{\infty}\left([0, T] ; L^{\infty} \cap D^{2}\right) ; \\
(B) \quad & u \in C\left([0, T] ; H^{3}\right) \cap L^{2}\left([0, T] ; H^{4}\right), \quad u_{t} \in C\left([0, T] ; H^{1}\right) \cap L^{2}\left([0, T] ; D^{2}\right), \\
& \rho^{\frac{\delta-1}{2}} \nabla u \in C\left([0, T] ; L^{2}\right), \quad \rho^{\frac{\delta-1}{2}} \nabla u_{t} \in L^{\infty}\left(\left[0, T_{*}\right] ; L^{2}\right), \\
& \rho^{\delta-1} \nabla u \in L^{\infty}\left([0, T] ; D^{1}\right), \quad \rho^{\delta-1} \nabla^{2} u \in C\left([0, T] ; H^{1}\right) \cap L^{2}\left([0, T] ; D^{2}\right) .
\end{aligned}
$$

Remark 1.1. First, it follows from the Definition 1.1 that $\nabla \rho^{\delta-1} \in L^{\infty}$, which means that the vacuum occurs if and only in the far field.

Second, we introduce some physical quantities that will be used in this paper:

$$
\begin{aligned}
m(t) & =\int \rho(t, x) \quad \text { (total mass), } \\
\mathbb{P}(t) & =\int \rho(t, x) u(t, x) \quad \text { (momentum), } \\
E_{k}(t) & =\frac{1}{2} \int \rho(t, x)|u(t, x)|^{2} \quad \text { (total kinetic energy), } \\
E(t) & =E_{k}(t)+\int \frac{P}{\gamma-1} \quad \text { (total energy). }
\end{aligned}
$$

Actually, it follows from the definition that a regular solution satisfies the conservation of total mass and momentum (see Lemma 4.2). Furthermore, it satisfies the energy equality (see (4.5)). Note that the conservation of momentum is not clear for the strong solution with vacuum to the flows of constant viscosities [5]. In this sense, the definition of regular 
solutions here is consistent with the physical background of the compressible Navier-Stokes equations.

The regular solutions select velocity in a physically reasonable way when the density approaches the vacuum at far fields. Under the help of this notion of solutions, the momentum equations can be reformulated into a special quasi-linear parabolic system with some possible singular source terms near the vacuum, and the coefficients in front of Lamé operator $L$ will tend to $\infty$ as $\rho \rightarrow 0$ in the far filed. However, the problem becomes trackable through an elaborate linearization and approximation process.

Now we are ready to state the main results in this paper. First we prove the existence of the unique regular solution with $\nabla \rho^{\delta-1} \in C\left([0, T] ; D^{1} \cap D^{2}\right)$ to (1.1)-(1.7).

Theorem 1.1. Let parameters $(\gamma, \delta, \alpha, \beta)$ satisfy

$$
\gamma>1, \quad 0<\delta<1, \quad \alpha>0, \quad 2 \alpha+3 \beta \geq 0 .
$$

If the initial data $\left(\rho_{0}, u_{0}\right)$ satisfies

$$
\rho_{0}>0, \quad\left(\rho_{0}^{\gamma-1}, u_{0}\right) \in H^{3}, \quad \nabla \rho_{0}^{\delta-1} \in D^{1} \cap D^{2}, \quad \nabla \rho_{0}^{\frac{\delta-1}{2}} \in L^{4},
$$

and the initial compatibility conditions:

$$
\nabla u_{0}=\rho_{0}^{\frac{1-\delta}{2}} g_{1}, \quad L u_{0}=\rho_{0}^{1-\delta} g_{2}, \quad \nabla\left(\rho_{0}^{\delta-1} L u_{0}\right)=\rho_{0}^{\frac{1-\delta}{2}} g_{3},
$$

for some $\left(g_{1}, g_{2}, g_{3}\right) \in L^{2}$, then there exist a time $T_{*}>0$ and a unique regular solution $(\rho, u)$ in $\left[0, T_{*}\right] \times \mathbb{R}^{3}$ to the Cauchy problem (1.1)-(1.7) satisfying:

$$
\begin{aligned}
& t^{\frac{1}{2}} u \in L^{\infty}\left(\left[0, T_{*}\right] ; D^{4}\right), \quad t^{\frac{1}{2}} u_{t} \in L^{\infty}\left(\left[0, T_{*}\right] ; D^{2}\right) \cap L^{2}\left(\left[0, T_{*}\right] ; D^{3}\right), \\
& u_{t t} \in L^{2}\left(\left[0, T_{*}\right] ; L^{2}\right), \quad t^{\frac{1}{2}} u_{t t} \in L^{\infty}\left(\left[0, T_{*}\right] ; L^{2}\right) \cap L^{2}\left(\left[0, T_{*}\right] ; D^{1}\right), \\
& \rho^{1-\delta} \in L^{\infty}\left(\left[0, T_{*}\right] ; L^{\infty} \cap D^{1,6} \cap D^{2,3} \cap D^{3}\right), \\
& \nabla \rho^{\delta-1} \in C\left([0, T] ; D^{1} \cap D^{2}\right), \quad \nabla \log \rho \in L^{\infty}\left(\left[0, T_{*}\right] ; L^{\infty} \cap L^{6} \cap D^{1,3} \cap D^{2}\right) .
\end{aligned}
$$

Moreover, if $1<\gamma \leq 2,(\rho, u)$ is a classical solution to (1.1)-1.7) in $\left(0, T_{*}\right] \times \mathbb{R}^{3}$.

Remark 1.2. The conditions (1.12)-(1.13) in Theorem 1.1 identify a class of admissible initial data that makes the problem (1.1) -(1.7) solvable, which are satisfied by, for example,

$$
\rho_{0}(x)=\frac{1}{1+|x|^{2 a}}, \quad u_{0}(x) \in C_{0}^{3}\left(\mathbb{R}^{3}\right), \quad \text { and } \quad \frac{3}{4(\gamma-1)}<a<\frac{1}{4(1-\delta)} .
$$

Particularly, when $\nabla u_{0}$ is compactly supported, the compatibility conditions (1.13) are satisfied automatically.

Second, we can also prove the existence of the unique regular solution with $\nabla \rho^{\delta-1} \in$ $C\left([0, T] ; L^{q} \cap D^{1,3} \cap D^{2}\right)$ to (1.1)-(1.7).

Theorem 1.2. Let (1.11) hold, and $q \in(3,+\infty)$ be a fixed constant. If the initial data $\left(\rho_{0}, u_{0}\right)$ satisfies

$$
\rho_{0}>0, \quad\left(\rho_{0}^{\gamma-1}, u_{0}\right) \in H^{3}, \quad \nabla \rho_{0}^{\delta-1} \in L^{q} \cap D^{1,3} \cap D^{2}, \quad \nabla \rho_{0}^{\frac{\delta-1}{2}} \in L^{6},
$$


and the initial compatibility conditions (1.13), then there exist a time $T_{*}>0$ and a unique regular solution $(\rho, u)$ in $\left[0, T_{*}\right] \times \mathbb{R}^{3}$ to the Cauchy problem (1.1)-(1.7) satisfying:

$$
\begin{aligned}
& t^{\frac{1}{2}} u \in L^{\infty}\left(\left[0, T_{*}\right] ; D^{4}\right), \quad t^{\frac{1}{2}} u_{t} \in L^{\infty}\left(\left[0, T_{*}\right] ; D^{2}\right) \cap L^{2}\left(\left[0, T_{*}\right] ; D^{3}\right), \\
& u_{t t} \in L^{2}\left(\left[0, T_{*}\right] ; L^{2}\right), \quad t^{\frac{1}{2}} u_{t t} \in L^{\infty}\left(\left[0, T_{*}\right] ; L^{2}\right) \cap L^{2}\left(\left[0, T_{*}\right] ; D^{1}\right), \\
& \rho^{1-\delta} \in L^{\infty}\left(\left[0, T_{*}\right] ; L^{\infty} \cap D^{1, q} \cap D^{1,6} \cap D^{1, \infty} \cap D^{2,3} \cap D^{3}\right), \\
& \nabla \rho^{\delta-1} \in C\left([0, T] ; L^{q} \cap D^{1,3} \cap D^{2}\right), \\
& \nabla \log \rho \in L^{\infty}\left(\left[0, T_{*}\right] ; L^{q} \cap L^{6} \cap L^{\infty} \cap D^{1,3} \cap D^{2}\right) .
\end{aligned}
$$

Moreover, if $1<\gamma \leq 2,(\rho, u)$ is a classical solution to (1.1)-(1.7) in $\left(0, T_{*}\right] \times \mathbb{R}^{3}$.

Remark 1.3. The conditions (1.15) and (1.13) in Theorem 1.2 identify a class of admissible initial data that makes the problem (1.1)-(1.7) solvable, which are satisfied by, for example,

$$
\rho_{0}(x)=\frac{1}{1+|x|^{2 a}}, \quad u_{0}(x) \in C_{0}^{3}\left(\mathbb{R}^{3}\right), \quad \text { and } \quad \frac{3}{4(\gamma-1)}<a<\frac{1-3 / q}{2(1-\delta)} .
$$

It should be pointed out that, for such kind of initial data, when $q$ is chosen large enough, the range of a is wider than that of a shown in the example of Remark 1.2. However, for general initial data, the conclusion obtained in any one of Theorems 1.1-1.2 cannot be implied by that of the other one.

Remark 1.4. The compatibility conditions (1.13) are also necessary for the existence of regular solutions $(\rho, u)$ obtained in Theorems 1.1 1.2. In particular, the one shown in $(1.13)_{2}\left([1.13)_{3}\right)$ plays a key role in the derivation of $u_{t} \in L^{\infty}\left(\left[0, T_{*}\right] ; L^{2}\left(\mathbb{R}^{3}\right)\right)\left(\rho^{\frac{\delta-1}{2}} \nabla u_{t} \in\right.$ $\left.L^{\infty}\left(\left[0, T_{*}\right] ; L^{2}\left(\mathbb{R}^{3}\right)\right)\right)$, which will be used in the uniform estimates for $|u|_{D^{2}}\left(|u|_{D^{3}}\right)$.

A natural question is whether the local solution in Theorems 1.1.1.2 can be extended globally in time, and what the large time behavior is. In contrast to the classical theory for the constant viscosity case [10, 22, 28], we show the following somewhat surprising phenomenon that such an extension is impossible if the velocity field decays to zero as $t \rightarrow$ $+\infty$ and the initial total momentum is non-zero. First, based on the physical quantities introduced in Remark 1.1, we define a solution class as follows:

Definition 1.2. Let $T>0$ be any constant. For the Cauchy problem (1.1)-(1.7), a classical solution $(\rho, u)$ is said to be in $D(T)$ if $(\rho, u)$ satisfies the following conditions:

(A) Conservation of total mass: $0<m(0)=m(t)<\infty$ for any $t \in[0, T]$;

(B) Conservation of momentum: $0<|\mathbb{P}(0)|=|\mathbb{P}(t)|<+\infty$ for any $t \in[0, T]$;

(C) Finite kinetic energy: $0<E_{k}(t)<\infty$ for any $t \in[0, T]$.

Then one has:

Theorem 1.3. Let parameters $(\gamma, \delta, \alpha, \beta)$ satisfy

$$
\gamma \geq 1, \quad \delta \geq 0, \quad \alpha>0, \quad 2 \alpha+3 \beta \geq 0 .
$$

Then for the Cauchy problem (1.1)-(1.7), there is no classical solution $(\rho, u) \in D(\infty)$ with

$$
\limsup _{t \rightarrow+\infty}|u(t, x)|_{\infty}=0 \text {. }
$$


According to Theorems 1.1, 1.3 and Remark 1.1, one shows that

Corollary 1.1. Let (1.11) hold and

$$
1<\gamma \leq \frac{3}{2}, \quad \delta \in[\gamma-1,1)
$$

Assume that $m(0)>0$ and $|\mathbb{P}(0)|>0$. Then for the Cauchy problem (1.1)-(1.7), there is no global regular solution $(\rho, u)$ defined in Definition 1.1 satisfying the following decay

$$
\limsup _{t \rightarrow+\infty}|u(t, x)|_{\infty}=0 \text {. }
$$

Moreover, according to Theorem[1.3, Remark 1.1 and [5, 6, 10, for constant viscosity flow, one can give the following example: the classical solution exists globally and keeps the conservation of total mass, but can not keep the conservation of momentum for all the time $t \in(0, \infty)$.

Corollary 1.2. Let $\delta=0$ in (1.4). For any given numbers $M>0, \iota \in\left(\frac{1}{2}, 1\right]$, and $\bar{\rho}>0$, suppose that the initial data $\left(\rho_{0}, u_{0}\right)$ satisfies

$$
\begin{aligned}
& E(0)<\infty, \quad u_{0} \in \dot{H}^{\iota} \cap D^{1} \cap D^{3}, \quad \rho_{0}^{\frac{1}{2}} \in H^{1}, \\
& \left(\rho_{0}, P\left(\rho_{0}\right)\right) \in H^{3}, \quad 0 \leq \inf \rho_{0} \leq \sup \rho_{0} \leq \bar{\rho}, \quad\|u\|_{\dot{H}^{\iota}} \leq M,
\end{aligned}
$$

and the compatibility condition

$$
-\alpha \triangle u_{0}-(\alpha+\beta) \nabla \text { divu }_{0}+\nabla P\left(\rho_{0}\right)=\rho_{0} g_{4},
$$

for some $g_{4} \in D^{1}$ with $\rho^{\frac{1}{2}} g_{4} \in L^{2}$. Then there exists a positive constant $\zeta$ depending on $\alpha, \beta, A, \gamma, \bar{\rho}, \iota$ and $M$ such that if

$$
E(0) \leq \zeta,
$$

the Cauchy problem (1.1)-1.7) has a unique global classical solution $(\rho, u)$ in $(0, \infty) \times \mathbb{R}^{3}$ satisfying, for any $0<\tau<T<\infty$,

$$
\begin{aligned}
& E(t) \leq E(0), \quad t \in[0, \infty) ; \quad 0 \leq \rho(t, x) \leq 2 \bar{\rho}, \quad(t, x) \in[0, \infty) \times \mathbb{R}^{3} ; \\
& \rho^{\frac{1}{2}} \in C\left([0, T] ; H^{1}\right) ; \quad m(t)=m(0), \quad t \in[0, \infty) ; \\
& \sup _{t \in[0, T]}(\min \{1, t\})^{3} \int \rho|\dot{u}|^{2} \leq 2 E(0)^{\frac{1}{2}} ; \quad(\rho, P(\rho)) \in C\left([0, T] ; H^{3}\right) ; \\
& u \in C\left([0, T] ; D^{1} \cap D^{3}\right) \cap L^{2}\left([0, T] ; D^{4}\right) \cap L^{\infty}\left([\tau, T] ; D^{4}\right) ; \\
& u_{t} \in L^{\infty}\left([0, T] ; D^{1}\right) \cap L^{2}\left([0, T] ; D^{2}\right) \cap L^{\infty}\left([\tau, T] ; D^{2}\right) \cap H^{1}\left([\tau, T] ; D^{1}\right),
\end{aligned}
$$

for $\dot{u}=u_{t}+u \cdot \nabla u$, and the following large-time behavior

$$
\lim _{t \rightarrow \infty} \int\left(|\rho|^{b}+\rho^{\frac{1}{2}}|u|^{4}+|\nabla u|^{2}\right)(t, x) d x=0, \quad \text { for any constant } b>\gamma .
$$

Furthermore, if $m(0)>0$ and $|\mathbb{P}(0)|>0$, then the solution obtained above can not keep the conservation of the momentum for all the time $t \in(0, \infty)$. 
Remark 1.5. Note that the classes of initial data for Corollary 1.2 is a sub-class of initial data for the global well-posedness theory in [10]. Indeed, compared with the assumptions on initial data in [10], additional conditions,

$$
\rho_{0}^{\frac{1}{2}} \in H^{1}, \quad m(0)>0, \quad \text { and } \quad|\mathbb{P}(0)|>0,
$$

are required here to keep the conservation of positive total mass, and satisfy the assumptions of Theorem 1.3.

Remark 1.6. Note that for the regular solution $(\rho, u)$ obtained in Theorems 1.1-1.2, $u$ stays in the inhomogeneous Sobolev space $H^{3}$ instead of the homogenous one $D^{1} \cap D^{3}$ in [5] for constant viscous flows.

It is also worth pointing out that recently, if the initial density is compactly supported, Li-Wang-Xin [14] prove that classical solutions with finite energy to the Cauchy problem of the compressible Navier-Stokes systems with constant viscosities do not exist in general in inhomogeneous Sobolev space for any short time, which indicates in particular that the homogeneous Sobolev space is crucial as studying the well-posedness (even locally in time) for the Cauchy problem of the compressible Navier-Stokes systems in the presence of such kind of the vacuum.

Based on the conclusion obtained in Theorems 1.1]1.2 and 14], first there is a natural question that whether one can obtain the local-in-time existence of the classical solutions with finite energy in inhomogeneous Sobolev space to the Cauchy problem of the compressible Navier-Stokes systems with constant viscosities under the assumption that the initial density is positive but decays to zero in the far field, or not. Second, can the conclusion obtained in [14] be applied to the degenerate system considered here? Due to the obvious difference on the structure between the constant viscous flows and degenerate viscous flows, such questions are not easy and will be discussed in our future work Xin-Zhu [30].

Remark 1.7. Under the assumption inf $\rho_{0}=0$, in Li-Xin [15, the global existence of weak solutions to the Cauchy problem (1.1)-(1.7) has been established for the cases $3 / 4<$ $\delta<1,1<\gamma<6 \delta-3$ in the three dimensional space, and $1 / 2<\delta<1, \gamma>1$ in the two dimensional space.

We now outline the organization of the rest of this paper. In Section 2, we list some basic lemmas to be used later.

Section 3 is devoted to proving Theorem 1.1. First, in order to analyze the behavior of the possible singular term $\nabla \rho^{\delta-1}$ clearly, we enlarge the original problem (1.1)-(1.7) into (3.2)- (3.5) in terms of the following variables

$$
\phi=\frac{A \gamma}{\gamma-1} \rho^{\gamma-1}, \quad \psi=\frac{\delta}{\delta-1} \nabla \rho^{\delta-1}=\frac{\delta}{\delta-1}\left(\frac{A \gamma}{\gamma-1}\right)^{\frac{1-\delta}{\gamma-1}} \nabla \phi^{\frac{\delta-1}{\gamma-1}}=\left(\psi^{(1)}, \psi^{(2)}, \psi^{(3)}\right), \quad u
$$

in $\S 3.1$. Then the behavior of the velocity $u$ can be controlled by the following equations:

$$
u_{t}+u \cdot \nabla u+\nabla \phi+a \phi^{2 e} L u=\psi \cdot Q(u),
$$

where $a=\left(\frac{A \gamma}{\gamma-1}\right)^{-2 e}$, and $e=\frac{\delta-1}{2(\gamma-1)}<0$. Due to the fact that $\phi^{2 e}$ has an uniformly positive lower bound in the whole space, then for this special quasi-linear parabolic system, one can find formally that, even though the coefficients $a \phi^{2 e}$ in front of Lamé operator $L$ will tend to $\infty$ as $\rho \rightarrow 0$ in the far filed, yet this structure could give a better a priori 
estimate on $u$ in $H^{3}$ than those of [5, 18, 19, 29] if one can control the possible singular term $\psi$ in $D^{1} \cap D^{2}$. According to (1.1) 2 and (3.9), $\psi$ is governed by

$$
\psi_{t}+\sum_{l=1}^{3} A_{l}(u) \partial_{l} \psi+B(u) \psi+\delta a \phi^{2 e} \nabla \operatorname{div} u=0,
$$

where the definitions of $A_{l}(l=1,2,3)$ and $B$ can be found in $\S 3.2 .1$. This implies that actually the subtle source term $\psi$ could be controlled by a symmetric hyperbolic system with a possible singular higher order term $\delta a \phi^{2 e} \nabla \operatorname{div} u$. In order to close the estimates, we need to control $\phi^{2 e} \nabla \operatorname{div} u$ in $D^{1} \cap D^{2}$, which can be obtained by regarding the momentum equations as the following inhomogeneous Lamé equations:

$$
a L\left(\phi^{2 e} u\right)=-u_{t}-u \cdot \nabla u-\nabla \phi+\psi \cdot Q(u)-\frac{\delta-1}{\delta} G(\psi, u)=W,
$$

with

$$
G(\psi, u)=\alpha \psi \cdot \nabla u+\alpha \operatorname{div}(u \otimes \psi)+(\alpha+\beta)(\psi \operatorname{div} u+\psi \cdot(\nabla u)+u \cdot \nabla \psi) .
$$

In fact, one has

$$
\begin{aligned}
\left|\phi^{2 e} \nabla^{2} u\right|_{D^{1}} & \leq C\left(|\psi|_{\infty}\left|\nabla^{2} u\right|_{2}+\left|\phi^{2 e} \nabla^{3} u\right|_{2}\right) \\
& \leq C\left(|\psi|_{\infty}\left|\nabla^{2} u\right|_{2}+|\nabla \psi|_{3}|\nabla u|_{6}+\left|\nabla^{2} \psi\right|_{2}|u|_{\infty}+|W|_{D^{1}}\right),
\end{aligned}
$$

for some constant $C>0$ independent of the lower bound of the density provided that

$$
\phi^{2 e} u \rightarrow 0 \quad \text { as } \quad|x| \rightarrow+\infty,
$$

which can be verified in an approximation process from non-vacuum flows to the flow with far field vacuum. Similar calculations can be done for $\left|\phi^{2 e} \nabla^{2} u\right|_{D^{2}}$. Thus, it seems that at least, the reformulated system (3.2):

$$
\left\{\begin{array}{l}
\phi_{t}+u \cdot \nabla \phi+(\gamma-1) \phi \operatorname{div} u=0, \\
u_{t}+u \cdot \nabla u+\nabla \phi+a \phi^{2 e} L u=\psi \cdot Q(u), \\
\psi_{t}+\nabla(u \cdot \psi)+(\delta-1) \psi \operatorname{div} u+\delta a \phi^{2 e} \nabla \operatorname{div} u=0,
\end{array}\right.
$$

can provide a closed a priori estimates for the desired regular solution. Next we need to find a proper linear scheme to verify the energy estimate strategy discussed above.

Second, in $\S 3.2$, we give an elaborate linearization (3.15) of the nonlinear one (3.2)(3.5) based on a careful analysis on the structure of the nonlinear equations (3.2), and the global approximate solutions for this linearized problem when $\phi(0, x)=\phi_{0}$ has positive lower bound $\eta$ are established. The choice of the linear scheme for this problem needs to be careful due to the appearance of the far field vacuum. Some necessary structures should be preserved in order to establish the desired a priori estimates according to the strategy mentioned in the above paragraph. For the problem (3.2), a crucial point is how to deal with the estimates on $\psi$. According to the analysis in the above paragraph, we need to keep the two factors $\phi^{2 e}$ and $\nabla \operatorname{div} u$ of the source term $\delta a \phi^{2 e} \nabla \operatorname{div} u$ in equations (1.29) in the same step. Then let $v=\left(v^{(1)}, v^{(2)}, v^{(3)}\right) \in \mathbb{R}^{3}$ be a known vector and $g$ be a 
known real (scalar) function satisfying $(v(0, x), g(0, x))=\left(u_{0},\left(\phi_{0}\right)^{2 e}\right)$ and (3.16) $)$. It seems that one should consider the following linear equations:

$$
\left\{\begin{array}{l}
\phi_{t}+v \cdot \nabla \phi+(\gamma-1) \phi \operatorname{div} v=0, \\
u_{t}+v \cdot \nabla v+\nabla \phi+a \phi^{2 e} L u=\psi \cdot Q(v), \\
\psi_{t}+\sum_{l=1}^{3} A_{l}(v) \partial_{l} \psi+B(v) \psi+\delta a g \nabla \operatorname{div} v=0 .
\end{array}\right.
$$

However, it should be pointed out that, in (1.31), the relationship

$$
\psi=\frac{a \delta}{\delta-1} \nabla \phi^{2 e}
$$

between $\psi$ and $\phi$ has been destroyed due to the term $g \nabla \operatorname{div} v$ in (1.31) 3 . Then the estimates for the linear scheme (1.31) encounter an obvious difficulty when one considers the $L^{2}$ estimate on $u$ :

$$
\begin{aligned}
& \frac{1}{2} \frac{d}{d t}|u|_{2}^{2}+a \alpha\left|\phi^{e} \nabla u\right|_{2}^{2}+a(\alpha+\beta)\left|\phi^{e} \operatorname{div} u\right|_{2}^{2} \\
= & -\int(v \cdot \nabla v+\nabla \phi+a \underbrace{\nabla \phi^{2 e}}_{\neq \psi} \cdot Q(u)-\psi \cdot Q(v)) \cdot u .
\end{aligned}
$$

The factor $\nabla \phi^{2 e}$ does not coincide with $\frac{\delta-1}{a \delta} \psi$ in this linear scheme, which means that there is no way to control the term $a \nabla \phi^{2 e} \cdot Q(u)$ in the above energy estimates. In order to overcome this difficulty, in (3.15), we first linearize the equation of $h=\phi^{2 e}$ as:

$$
h_{t}+v \cdot \nabla h+(\delta-1) g \operatorname{div} v=0,
$$

and then use $h$ to define $\psi=\frac{a \delta}{\delta-1} \nabla h$ again. The linearized equations for $u$ are chosen as

$$
u_{t}+v \cdot \nabla v+\nabla \phi+a \sqrt{h^{2}+\epsilon^{2}} L u=\psi \cdot Q(v)
$$

for any positive constant $\epsilon>0$. Here the appearance of $\epsilon$ is used to compensate the lack of lower bound of $h$. It follows from (1.33) and the relation $\psi=\frac{a \delta}{\delta-1} \nabla h$ that

$$
\psi_{t}+\sum_{l=1}^{3} A_{l}(v) \partial_{l} \psi+(\nabla v)^{\top} \psi+a \delta(g \nabla \operatorname{div} v+\nabla g \operatorname{div} v)=0
$$

which turns out to be enough to get desired estimates on $\psi$.

In $\S 3.3$, the a priori estimates independent of the lower bound $(\epsilon, \eta)$ of the solutions $\left(\phi^{\epsilon, \eta}, h^{\epsilon, \eta}, \psi^{\epsilon, \eta}, u^{\epsilon, \eta}\right)$ to the linearized problem (3.15) are established. In order to deal with the limit process from our linear problem to the nonlinear one, we need some uniform estimates on the following new quantities:

$$
\varphi=h^{-1}, \quad f=\psi \varphi=\frac{a \delta}{\delta-1} \nabla h / h=\left(f^{(1)}, f^{(2)}, f^{(3)}\right) .
$$

An observation used in this subsection is that the initial assumption (3.6) implies that

$$
\varphi(0, x) \in L^{\infty} \cap D^{1,6} \cap D^{2,3} \cap D^{3}, \quad f(0, x) \in L^{\infty} \cap L^{6} \cap D^{1,3} \cap D^{2} .
$$


According to the definitions of $(\varphi, f)$ and the equation of $h$, one can also show that $(\varphi, f)$ can be controlled by some hyperbolic equations without degenerate or singular source terms. Based on these facts, we can obtain some uniform estimates on $\varphi$ and $f$, which are sufficient for the strong compactness argument used in §3.5. In $\S 3.4$, one obtains the uniformly local-in-time well-posedness of the linearized problem when $\phi_{0}>\eta>0$ but without the artificial viscosity by passing to the limit $\epsilon \rightarrow 0$.

In $\S 3.5$, based on the above analysis for the choice of the linearization and a new formulation of our problem (see (3.93)), the unique solvability of the classical solution away from vacuum to the nonlinear reformulated problem (3.2)-(3.5) through an iteration process is given, whose life span is uniformly positive with respect to the lower bound $\eta$ of $\phi_{0}$. Actually, the behavior of $u$ in this subsection is controlled by the following linear equations:

$$
\varphi\left(u_{t}+v \cdot \nabla v+\nabla \phi\right)+a L u=f \cdot Q(v),
$$

which is also a special quasi-linear parabolic system with some possible singular source terms $f \cdot Q(u)$ near the vacuum, and the coefficients $\varphi$ in front of the time evolution operator $\dot{u}=u_{t}+u \cdot \nabla u$ will tend to 0 as $\varphi \rightarrow 0$ in the far filed. However, based on the uniform estimates on $(\phi, u, h, \psi)$ and $(\varphi, f)$ established in $\S 3.4$, this structure can avoid some difficulties on the strong convergence of terms such as $g \nabla \operatorname{div} v$, and $h L u$ in the linear scheme (3.15). The details can be found in the proof of this subsection.

Based on the conclusions of $\S 3.5$, one can recover the solution of the nonlinear reformulated problem allowing vacuum in the far field by passing to the limit as $\eta \rightarrow 0$ in $\S 3.6$. Then in $\S 3.7$, one can show that the existence result for the reformulated problem indeed implies Theorem 1.1. Theorem 1.2 can be proved by a similar argument as used in Theorem 1.1.

Finally, Section 4 is devoted to the proof of the non-existence theories of global regular solutions with $L^{\infty}$ decay on $u$ shown in Theorem 1.3 and Corollaries 1.1.1.2. Furthermore, it should be pointed out that our framework in this paper can be applied to other physical dimensions, say 1 and 2 , with some minor modifications.

\section{Preliminaries}

In this section, we list some basic lemmas to be used later. The first one is the wellknown Gagliardo-Nirenberg inequality.

Lemma 2.1. [13. For $p \in[2,6], q \in(1, \infty)$, and $r \in(3, \infty)$, there exists some generic constant $C>0$ that may depend on $q$ and $r$ such that for

$$
f \in H^{1}\left(\mathbb{R}^{3}\right), \quad \text { and } \quad g \in L^{q}\left(\mathbb{R}^{3}\right) \cap D^{1, r}\left(\mathbb{R}^{3}\right),
$$

it holds that

$$
|f|_{p}^{p} \leq C|f|_{2}^{(6-p) / 2}|\nabla f|_{2}^{(3 p-6) / 2}, \quad|g|_{\infty} \leq C|g|_{q}^{q(r-3) /(3 r+q(r-3))}|\nabla g|_{r}^{3 r /(3 r+q(r-3))} .
$$

Some special cases of this inequality are

$$
|u|_{6} \leq C|u|_{D^{1}}, \quad|u|_{\infty} \leq C|u|_{6}^{\frac{1}{2}}|\nabla u|_{6}^{\frac{1}{2}}, \quad|u|_{\infty} \leq C\|u\|_{W^{1, r}} .
$$

The second lemma gives some compactness results obtained via the Aubin-Lions Lemma.

Lemma 2.2. 26] Let $X_{0} \subset X \subset X_{1}$ be three Banach spaces. Suppose that $X_{0}$ is compactly embedded in $X$ and $X$ is continuously embedded in $X_{1}$. Then the following statements hold. 
i) If $J$ is bounded in $L^{p}\left([0, T] ; X_{0}\right)$ for $1 \leq p<+\infty$, and $\frac{\partial J}{\partial t}$ is bounded in $L^{1}\left([0, T] ; X_{1}\right)$, then $J$ is relatively compact in $L^{p}([0, T] ; X)$;

ii) If $J$ is bounded in $L^{\infty}\left([0, T] ; X_{0}\right)$ and $\frac{\partial J}{\partial t}$ is bounded in $L^{p}\left([0, T] ; X_{1}\right)$ for $p>1$, then $J$ is relatively compact in $C([0, T] ; X)$.

The third one can be found in Majda [21].

Lemma 2.3. 21] Let $r, a$ and $b$ be constants such that

$$
\frac{1}{r}=\frac{1}{a}+\frac{1}{b}, \quad \text { and } \quad 1 \leq a, b, r \leq \infty .
$$

$\forall s \geq 1$, if $f, g \in W^{s, a} \cap W^{s, b}\left(\mathbb{R}^{3}\right)$, then it holds that

$$
\begin{aligned}
& \left|\nabla^{s}(f g)-f \nabla^{s} g\right|_{r} \leq C_{s}\left(|\nabla f|_{a}\left|\nabla^{s-1} g\right|_{b}+\left|\nabla^{s} f\right|_{b}|g|_{a}\right), \\
& \left|\nabla^{s}(f g)-f \nabla^{s} g\right|_{r} \leq C_{s}\left(|\nabla f|_{a}\left|\nabla^{s-1} g\right|_{b}+\left|\nabla^{s} f\right|_{a}|g|_{b}\right),
\end{aligned}
$$

where $C_{s}>0$ is a constant depending only on $s$, and $\nabla^{s} f(s>1)$ is the set of all $\partial_{x}^{\zeta} f$ with $|\zeta|=$ s. Here $\zeta=\left(\zeta_{1}, \zeta_{2}, \zeta_{3}\right) \in \mathbb{R}^{3}$ is a multi-index.

The following lemma is important in the derivation of the a priori estimates in Section 3 , which can be found in Remark 1 of [1].

Lemma 2.4. [1] If $f(t, x) \in L^{2}\left([0, T] ; L^{2}\right)$, then there exists a sequence $s_{k}$ such that

$$
s_{k} \rightarrow 0, \quad \text { and } \quad s_{k}\left|f\left(s_{k}, x\right)\right|_{2}^{2} \rightarrow 0, \quad \text { as } k \rightarrow+\infty .
$$

The following regularity estimate for the Lamé operator is standard in harmonic analysis.

Lemma 2.5. 27] If $u \in D^{1, q}\left(\mathbb{R}^{3}\right)$ with $1<q<+\infty$ is a weak solution to the problem

$$
\left\{\begin{array}{l}
-\alpha \triangle u-(\alpha+\beta) \nabla \text { divu }=L u=Z, \\
u \rightarrow 0 \quad \text { as } \quad|x| \rightarrow+\infty
\end{array}\right.
$$

then it holds that

$$
|u|_{D^{k+2, q}} \leq C|Z|_{D^{k, q}}
$$

where the constant $C>0$ depends only on $\alpha, \beta$ and $q$.

The final lemma is useful to improve a weak convergence to the strong convergence.

Lemma 2.6. [21] If the function sequence $\left\{w_{n}\right\}_{n=1}^{\infty}$ converges weakly to $w$ in a Hilbert space $X$, then it converges strongly to $w$ in $X$ if and only if

$$
\|w\|_{X} \geq \lim \sup _{n \rightarrow \infty}\left\|w_{n}\right\|_{X} .
$$

\section{LOCAL-IN-TIME WELL-POSEDNESS OF REGULAR SOLUTIONS}

This section is devoted to proving Theorem 1.1. To this end, we first reformulate the original Cauchy problem (1.1)-(1.7) as (3.2)-(3.5) below in terms of some new variables, and then establish the local well-posedness of the smooth solution to (3.2)-(3.5). In the end of this section, one can show that the existence result for the reformulated problem indeed implies Theorem 1.1 . 
3.1. Reformulation. In terms of variables

$$
\phi=\frac{A \gamma}{\gamma-1} \rho^{\gamma-1}, \quad \psi=\frac{\delta}{\delta-1} \nabla \rho^{\delta-1}=\frac{\delta}{\delta-1}\left(\frac{A \gamma}{\gamma-1}\right)^{\frac{1-\delta}{\gamma-1}} \nabla \phi^{\frac{\delta-1}{\gamma-1}}=\left(\psi^{(1)}, \psi^{(2)}, \psi^{(3)}\right)
$$

and $u$, the system (1.1) can be rewritten as

$$
\left\{\begin{array}{l}
\phi_{t}+u \cdot \nabla \phi+(\gamma-1) \phi \operatorname{div} u=0, \\
u_{t}+u \cdot \nabla u+\nabla \phi+a \phi^{2 e} L u=\psi \cdot Q(u), \\
\psi_{t}+\nabla(u \cdot \psi)+(\delta-1) \psi \operatorname{div} u+\delta a \phi^{2 e} \nabla \operatorname{div} u=0,
\end{array}\right.
$$

where

$$
a=\left(\frac{A \gamma}{\gamma-1}\right)^{\frac{1-\delta}{\gamma-1}}, \quad \text { and } \quad e=\frac{\delta-1}{2(\gamma-1)}<0 .
$$

The initial data is given by

$$
\left.(\phi, u, \psi)\right|_{t=0}=\left(\phi_{0}, u_{0}, \psi_{0}\right)=\left(\frac{A \gamma}{\gamma-1} \rho_{0}^{\gamma-1}(x), u_{0}(x), \frac{\delta}{\delta-1} \nabla \rho_{0}^{\delta-1}(x)\right), \quad x \in \mathbb{R}^{3} .
$$

$(\phi, u, \psi)$ is assumed to satisfy the far field behavior:

$$
(\phi, u, \psi) \rightarrow(0,0,0), \quad \text { as } \quad|x| \rightarrow+\infty, \quad t \geq 0 .
$$

To prove Theorem 1.1, our first step is to establish the following well-posedness to the reformulated problem (3.2)-(3.5).

Theorem 3.1. Let (1.11) hold. If the initial data $\left(\phi_{0}, u_{0}, \psi_{0}\right)$ satisfies:

$$
\phi_{0}>0, \quad\left(\phi_{0}, u_{0}\right) \in H^{3}, \quad \psi_{0} \in D^{1} \cap D^{2}, \quad \nabla \phi_{0}^{e} \in L^{4},
$$

and the initial compatibility conditions:

$$
\nabla u_{0}=\phi_{0}^{-e} g_{1}, \quad a L u_{0}=\phi_{0}^{-2 e} g_{2}, \quad \nabla\left(a \phi_{0}^{2 e} L u_{0}\right)=\phi_{0}^{-e} g_{3},
$$

for some $\left(g_{1}, g_{2}, g_{3}\right) \in L^{2}$, then there exist a time $T_{*}>0$ and a unique classical solution $\left(\phi, u, \psi=\frac{a \delta}{\delta-1} \nabla \phi^{2 e}\right)$ to the Cauchy problem (3.2)-(3.5), satisfying

$$
\begin{aligned}
& \phi \in C\left(\left[0, T_{*}\right] ; H^{3}\right), \quad \nabla \phi / \phi \in L^{\infty}\left(\left[0, T_{*}\right] ; L^{\infty} \cap L^{6} \cap D^{1,3} \cap D^{2}\right), \\
& \psi \in C\left(\left[0, T_{*}\right] ; D^{1} \cap D^{2}\right), \quad \phi^{-2 e} \in L^{\infty}\left(\left[0, T_{*}\right] ; L^{\infty} \cap D^{1,6} \cap D^{2,3} \cap D^{3}\right), \\
& u \in C\left(\left[0, T_{*}\right] ; H^{3}\right) \cap L^{2}\left(\left[0, T_{*}\right] ; H^{4}\right), \quad \phi^{2 e} \nabla u \in L^{\infty}\left(\left[0, T_{*}\right] ; D^{1}\right), \\
& \phi^{2 e} \nabla^{2} u \in C\left(\left[0, T_{*}\right] ; H^{1}\right), \quad \phi^{2 e} \nabla^{2} u \in L^{2}\left(\left[0, T_{*}\right] ; D^{2}\right), \phi^{e} \nabla u \in C\left(\left[0, T_{*}\right] ; L^{2}\right), \\
& \phi^{e} \nabla u_{t} \in L^{\infty}\left(\left[0, T_{*}\right] ; L^{2}\right), \quad u_{t} \in C\left(\left[0, T_{*}\right] ; H^{1}\right) \cap L^{2}\left(\left[0, T_{*}\right] ; D^{2}\right), \\
& \left(\phi^{2 e} \nabla^{2} u\right)_{t} \in L^{2}\left(\left[0, T_{*}\right] ; L^{2}\right), \quad u_{t t} \in L^{2}\left(\left[0, T_{*}\right] ; L^{2}\right), t^{\frac{1}{2}} u \in L^{\infty}\left(\left[0, T_{*}\right] ; D^{4}\right), \\
& t^{\frac{1}{2}} u_{t} \in L^{\infty}\left(\left[0, T_{*}\right] ; D^{2}\right) \cap L^{2}\left(\left[0, T_{*}\right] ; D^{3}\right), t^{\frac{1}{2}} u_{t t} \in L^{\infty}\left(\left[0, T_{*}\right] ; L^{2}\right) \cap L^{2}\left(\left[0, T_{*}\right] ; D^{1}\right) .
\end{aligned}
$$

This theorem will be proved in the subsequent four Subsections 3.2-3.6. 
3.2. Linearization. In order to solve the nonlinear problem (3.2)-(3.5), we need to consider the corresponding linearized problem. Before this, it is necessary to analyze the structure of the equations (3.2).

3.2.1. Structure of the nonlinear equations (3.2). First, due to the definition of $\psi$, if $\psi \in$ $D^{1,2}(K) \cap D^{2,2}(K)$ for any compact set $K \subset \mathbb{R}^{3}$, a direct calculation shows that

$$
\partial_{i} \psi^{(j)}=\partial_{j} \psi^{(i)} \quad \text { for } i, j=1,2,3
$$

in the sense of distributions. Thus, $(\underline{3.2})_{2}$ can be rewritten as

$$
\psi_{t}+\sum_{l=1}^{3} A_{l} \partial_{l} \psi+B \psi+\delta a \phi^{2 e} \nabla \operatorname{div} u=0,
$$

where $A_{l}=\left(a_{i j}^{l}\right)_{3 \times 3}$ for $i, j, l=1,2,3$, are symmetric with

$$
a_{i j}^{l}=u^{(l)} \quad \text { for } i=j ; \quad \text { otherwise } a_{i j}^{l}=0,
$$

and $B=(\nabla u)^{\top}+(\delta-1) \operatorname{div} u \mathbb{I}_{3}$. This implies that the subtle source term $\psi$ could be controlled by the symmetric hyperbolic system (3.9).

Second, for equations (3.2) 3 , note that the coefficients $a \phi^{2 e}$ in front of the Lamé operator $L$ will tend to $\infty$ as $\phi \rightarrow 0$ in the far filed. In order to make full use of this special structure, though system (3.2) for $(\phi, u, \psi)$ has been already a closed one, it is helpful to get some more precise estimates by introducing two auxiliary quantities:

$$
\varphi=\phi^{-2 e}, \quad f=\psi \varphi=\frac{2 a e \delta}{\delta-1} \frac{\nabla \phi}{\phi}=\left(f^{(1)}, f^{(2)}, f^{(3)}\right) .
$$

Next, we will show formally the time evolution mechanisms of $(\varphi, f)$ based on the initial regularities (3.6) and system (3.2). On the one hand, it follows from (3.1) and (3.10) that

$$
\psi_{0}=\frac{a \delta}{\delta-1} \nabla \varphi_{0}^{-1}=-\frac{a \delta}{\delta-1} \varphi_{0}^{-2} \nabla \varphi_{0}, \quad f_{0}=\varphi_{0} \psi_{0},
$$

which, along with (3.6), implies that

$$
\varphi_{0}=\phi_{0}^{-2 e} \in L^{\infty} \cap D^{1,6} \cap D^{2,3} \cap D^{3}, \quad f_{0}=\frac{2 a e \delta}{\delta-1} \nabla \phi_{0} / \phi_{0} \in L^{\infty} \cap L^{6} \cap D^{1,3} \cap D^{2} .
$$

On the other hand, it follows from (3.1) and equations (3.2) that

$$
\begin{aligned}
\varphi_{t}+u \cdot \nabla \varphi-(\delta-1) \varphi \operatorname{div} u & =0, \\
f_{t}+\nabla(u \cdot f)+a \delta \nabla \operatorname{div} u & =0 .
\end{aligned}
$$

If $f \in D^{1,2}(K) \cap D^{2,2}(K)$ for any compact set $K \subset \mathbb{R}^{3}$, a direct calculation shows that

$$
\partial_{i} f^{(j)}=\partial_{j} f^{(i)} \quad \text { for } i, j=1,2,3
$$

in the sense of distributions. Then $(\underline{3.13})_{2}$ can be written as

$$
f_{t}+\sum_{l=1}^{3} A_{l} \partial_{l} f+B^{*} f+a \delta \nabla \operatorname{div} u=0,
$$

where $B^{*}=(\nabla u)^{\top}$. Thus $f$ satisfies the symmetric hyperbolic system (3.14). 
It should be pointed out that a key observation here is that the structure in (3.13)(3.14) for $(\varphi, f)$ makes it possible to show that the subtle term $a \phi^{2 e} L u$ is well defined in $H^{2}$ when vacuum appears in the far field.

3.2.2. Linearized problem for uniformly positive initial density and artificial viscosity. Motivated by the above observations, we will consider the following linearized problem for $(\phi, u, h)$ :

$$
\left\{\begin{array}{l}
\phi_{t}+v \cdot \nabla \phi+(\gamma-1) \phi \operatorname{div} v=0, \\
u_{t}+v \cdot \nabla v+\nabla \phi+a \sqrt{h^{2}+\epsilon^{2}} L u=\psi \cdot Q(v), \\
h_{t}+v \cdot \nabla h+(\delta-1) g \operatorname{div} v=0, \\
\left.(\phi, u, h)\right|_{t=0}=\left(\phi_{0}, u_{0}, h_{0}\right)=\left(\phi_{0}, u_{0},\left(\phi_{0}\right)^{2 e}\right), \quad x \in \mathbb{R}^{3}, \\
(\phi, u, h) \rightarrow\left(\phi^{\infty}, 0, h^{\infty}\right), \quad \text { as } \quad|x| \rightarrow+\infty, \quad t \geq 0,
\end{array}\right.
$$

where $\epsilon$ and $\phi^{\infty}$ are both positive constants,

$$
h^{\infty}=\left(\phi^{\infty}\right)^{2 e}, \quad \psi=\frac{a \delta}{\delta-1} \nabla h,
$$

$v=\left(v^{(1)}, v^{(2)}, v^{(3)}\right) \in \mathbb{R}^{3}$ is a known vector and $g$ is a known real (scalar) function satisfying $(v(0, x), g(0, x))=\left(u_{0}, h_{0}\right)=\left(u_{0},\left(\phi_{0}\right)^{2 e}\right)$ and:

$$
\begin{aligned}
& g \in L^{\infty} \cap C\left([0, T] \times \mathbb{R}^{3}\right), \quad \nabla g \in C\left([0, T] ; H^{2}\right), \quad g_{t} \in C\left([0, T] ; H^{2}\right), \\
& \nabla g_{t t} \in L^{2}\left([0, T] ; L^{2}\right), \quad v \in C\left([0, T] ; H^{3}\right) \cap L^{2}\left([0, T] ; H^{4}\right), \quad t^{\frac{1}{2}} v \in L^{\infty}\left([0, T] ; D^{4}\right), \\
& v_{t} \in C\left([0, T] ; H^{1}\right) \cap L^{2}\left([0, T] ; D^{2}\right), \quad v_{t t} \in L^{2}\left([0, T] ; L^{2}\right), \\
& t^{\frac{1}{2}} v_{t} \in L^{\infty}\left([0, T] ; D^{2}\right) \cap L^{2}\left([0, T] ; D^{3}\right), \quad t^{\frac{1}{2}} v_{t t} \in L^{\infty}\left([0, T] ; L^{2}\right) \cap L^{2}\left([0, T] ; D^{1}\right),
\end{aligned}
$$

where $T>0$ is an arbitrary constant.

Now the following global well-posedness in $[0, T] \times \mathbb{R}^{3}$ of a classical solution to (3.15) can be obtained by the standard theory $([5,13,21])$ at least when $\phi_{0}$ is uniformly positive and $\epsilon>0$.

Lemma 3.1. Let (1.11) hold and $\epsilon>0$. Assume that $\left(\phi_{0}, u_{0}, h_{0}=\left(\phi_{0}\right)^{2 e}\right)$ satisfies

$$
\eta<\phi_{0}, \quad \phi_{0}-\phi^{\infty} \in H^{3}, \quad u_{0} \in H^{3},
$$

for some constant $\eta>0$. Then for any $T>0$, there exists a unique classical solution $(\phi, u, h)$ in $[0, T] \times \mathbb{R}^{3}$ to (3.15) such that

$$
\begin{aligned}
& \phi-\phi^{\infty} \in C\left([0, T] ; H^{3}\right), \quad h \in L^{\infty} \cap C\left([0, T] \times \mathbb{R}^{3}\right), \quad \nabla h \in C\left([0, T] ; H^{2}\right), \\
& h_{t} \in C\left([0, T] ; H^{2}\right), \quad u \in C\left([0, T] ; H^{3}\right) \cap L^{2}\left([0, T] ; H^{4}\right), \\
& u_{t} \in C\left([0, T] ; H^{1}\right) \cap L^{2}\left([0, T] ; D^{2}\right), \quad u_{t t} \in L^{2}\left([0, T] ; L^{2}\right), \quad t^{\frac{1}{2}} u \in L^{\infty}\left([0, T] ; D^{4}\right), \\
& t^{\frac{1}{2}} u_{t} \in L^{\infty}\left([0, T] ; D^{2}\right) \cap L^{2}\left([0, T] ; D^{3}\right), \quad t^{\frac{1}{2}} u_{t t} \in L^{\infty}\left([0, T] ; L^{2}\right) \cap L^{2}\left([0, T] ; D^{1}\right) .
\end{aligned}
$$

Remark 3.1. For the initial assumption on $h_{0}$, due to

$$
\eta<\phi_{0}, \quad \phi_{0}-\phi^{\infty} \in H^{3} \quad \text { and } \quad h_{0}=\left(\phi_{0}\right)^{2 e},
$$


it holds that

$$
h_{0} \in L^{\infty} \quad \text { and } \quad \nabla h_{0} \in H^{2} .
$$

Next we are going to establish the uniform a priori estimates independent of $(\epsilon, \eta)$ for the unique solution $(\phi, u, h)$ to (3.15) obtained in Lemma 3.1.

3.3. A priori estimates independent of $(\epsilon, \eta)$. Let $\left(\phi_{0}, u_{0}, h_{0}=\left(\phi_{0}\right)^{2 e}\right)$ be a given initial data satisfying the hypothesis of Lemma 3.1, and assume that there exists a constant $c_{0}>0$ independent of $\eta$ such that

$$
\begin{aligned}
I N_{0}= & 2+\phi^{\infty}+\left\|\phi_{0}-\phi^{\infty}\right\|_{3}+\left|\nabla h_{0}\right|_{D^{1} \cap D^{2}}+\left\|u_{0}\right\|_{3}+\left|g_{1}\right|_{2}+\left|g_{2}\right|_{2} \\
& +\left|g_{3}\right|_{2}+\left\|h_{0}^{-1}\right\|_{L^{\infty} \cap D^{1,6} \cap D^{2,3} \cap D^{3}}+\left\|\nabla h_{0} / h_{0}\right\|_{L^{\infty} \cap L^{6} \cap D^{1,3} \cap D^{2}} \leq c_{0},
\end{aligned}
$$

where

$$
g_{1}=\phi_{0}^{e} \nabla u_{0}, \quad g_{2}=a \phi_{0}^{2 e} L u_{0} \quad \text { and } \quad g_{3}=\phi_{0}^{e} \nabla\left(a \phi_{0}^{2 e} L u_{0}\right) .
$$

Remark 3.2. The choice of the constant $c_{0}$, independent of $\eta$, will be verified in the limit process from the non-vacuum problem to the one with far field vacuum in Subsection $\$ 3.6$ (see (3.131)).

Remark 3.3. First, it follows from (3.20), $\phi_{0}>\eta$ and the far field behavior of $(\phi, u, h)$ shown in (3.15) 5 that

$$
\left\{\begin{array}{l}
a L\left(\phi_{0}^{2 e} u_{0}\right)=g_{2}-\frac{\delta-1}{\delta} G\left(\psi_{0}, u_{0}\right), \\
\phi_{0}^{2 e} u_{0} \rightarrow 0 \quad \text { as } \quad|x| \rightarrow+\infty
\end{array}\right.
$$

where

$$
G=\alpha \psi_{0} \cdot \nabla u_{0}+\alpha \operatorname{div}\left(u_{0} \otimes \psi_{0}\right)+(\alpha+\beta)\left(\psi_{0} \operatorname{divu}_{0}+\psi_{0} \cdot\left(\nabla u_{0}\right)+u_{0} \cdot \nabla \psi_{0}\right)
$$

Then Lemma 2.5 and (3.19) imply that

$$
\begin{aligned}
\left|\phi_{0}^{2 e} u_{0}\right|_{D^{2}} \leq C\left(\left|g_{2}\right|_{2}+\left|G\left(\psi_{0}, u_{0}\right)\right|_{2}\right) & \leq C_{1}<+\infty \\
\left|\phi_{0}^{2 e} \nabla^{2} u_{0}\right|_{2} \leq C\left(\left|\phi_{0}^{2 e} u_{0}\right|_{D^{2}}+\left|\nabla \psi_{0}\right|_{6}\left|u_{0}\right|_{3}+\left|\psi_{0}\right|_{\infty}\left|\nabla u_{0}\right|_{2} \mid\right) & \leq C_{1}<+\infty
\end{aligned}
$$

where $C_{1}$ is a positive constant depending on $\left(c_{0}, A, \alpha, \beta, \gamma, \delta\right)$, but is independent of $(\epsilon, \eta)$.

Second, due to $\nabla^{2} \phi_{0}^{2 e} \in L^{2}$ and (3.22), one gets easily

$$
\left|\phi_{0}^{e} \nabla^{2} \phi_{0}\right|_{2}+\left|\phi_{0}^{e} \nabla\left(\psi_{0} \cdot Q\left(u_{0}\right)\right)\right|_{2} \leq C_{1}<+\infty .
$$

Now we fix $T>0$, and assume that there exist some time $T^{*} \in(0, T]$ and constants $c_{i}$ $(i=1, \ldots, 5)$ such that

$$
1<c_{0} \leq c_{1} \leq c_{2} \leq c_{3} \leq c_{4} \leq c_{5}
$$


and

$$
\begin{aligned}
& \sup _{0 \leq t \leq T^{*}}\|\nabla g(t)\|_{D^{1} \cap D^{2}}^{2} \leq c_{1}^{2}, \sup _{0 \leq t \leq T^{*}}\|v(t)\|_{1}^{2}+\int_{0}^{T^{*}}\left(|v|_{D^{2}}^{2}+\left|v_{t}\right|_{2}^{2}\right) \mathrm{d} t \leq c_{2}^{2}, \\
& \sup _{0 \leq t \leq T^{*}}\left(|v|_{D^{2}}^{2}+\left|v_{t}\right|_{2}^{2}+\left|g \nabla^{2} v\right|_{2}^{2}\right)(t)+\int_{0}^{T^{*}}\left(|v|_{D^{3}}^{2}+\left|v_{t}\right|_{D^{1}}^{2}\right) \mathrm{d} t \leq c_{3}^{2}, \\
& \sup _{0 \leq t \leq T^{*}}\left(|v|_{D^{3}}^{2}+\left|v_{t}\right|_{D^{1}}^{2}+\left|g_{t}\right|_{D^{1}}^{2}\right)(t)+\int_{0}^{T^{*}}\left(|v|_{D^{4}}^{2}+\left|v_{t}\right|_{D^{2}}^{2}+\left|v_{t t}\right|_{2}^{2}\right) \mathrm{d} t \leq c_{4}^{2}, \\
& \sup _{0 \leq t \leq T^{*}}\left(\left|g \nabla^{2} v\right|_{D^{1}}^{2}+\left|g_{t}\right|_{\infty}^{2}\right)(t)+\int_{0}^{T^{*}}\left(\left|\left(g \nabla^{2} v\right)_{t}\right|_{2}^{2}+\left|g \nabla^{2} v\right|_{D^{2}}^{2}\right) \mathrm{d} t \leq c_{4}^{2}, \\
& \operatorname{ess} \sup _{0 \leq t \leq T^{*}} t\left(\left|v_{t}\right|_{D^{2}}^{2}+|v|_{D^{4}}^{2}+\left|v_{t t}\right|_{2}^{2}\right)(t)+\int_{0}^{T^{*}} t\left(\left|v_{t t}\right|_{D^{1}}^{2}+\left|v_{t}\right|_{D^{3}}^{2}\right) \mathrm{d} t \leq c_{5}^{2} .
\end{aligned}
$$

$T^{*}$ and $c_{i}(i=1, \ldots, 5)$ will be determined later (see (3.79) $)$, and depend only on $c_{0}$ and the fixed constants $(A, \alpha, \beta, \gamma, \delta, T)$.

In the following we are going to establish a series of uniform local (in time) estimates independent of $(\epsilon, \eta)$ listed as Lemmas 3.2,3.7. Hereinafter, $C \geq 1$ will denote a generic positive constant depending only on fixed constants $(A, \alpha, \beta, \gamma, \delta, T)$.

\subsubsection{The a priori estimates for $\phi$. Now we estimate $\phi$.}

Lemma 3.2. Let $(\phi, u, h)$ be the unique classical solution to (3.15) in $[0, T] \times \mathbb{R}^{3}$. Then

$$
\begin{gathered}
\left\|\phi(t)-\phi^{\infty}\right\|_{3}^{2} \leq C c_{0}^{2}, \quad\left|\phi_{t}(t)\right|_{2} \leq C c_{0} c_{2}, \quad\left|\phi_{t}(t)\right|_{D^{1}} \leq C c_{0} c_{3}, \\
\left|\phi_{t}(t)\right|_{D^{2}} \leq C c_{0} c_{4}, \quad\left|\phi_{t t}(t)\right|_{2} \leq C c_{4}^{3}, \quad \int_{0}^{t}\left\|\phi_{t t}\right\|_{1}^{2} d s \leq C c_{0}^{2} c_{4}^{2},
\end{gathered}
$$

for $0 \leq t \leq T_{1}=\min \left(T^{*},\left(1+c_{4}\right)^{-2}\right)$.

Proof. First, the stand energy estimates argument for transport equations and (3.24) give

$$
\begin{aligned}
\left\|\phi(t)-\phi^{\infty}\right\|_{3} & \leq\left(\left\|\phi_{0}-\phi^{\infty}\right\|_{3}+\phi^{\infty} \int_{0}^{t}\|\nabla v\|_{3} \mathrm{~d} s\right) \exp \left(C \int_{0}^{t}\|v\|_{4} \mathrm{~d} s\right) \\
& \leq C c_{0} \quad \text { for } \quad 0 \leq t \leq T_{1}=\min \left(T^{*},\left(1+c_{4}\right)^{-2}\right) .
\end{aligned}
$$

Second, it follows from the equation (3.15) 1 and (2.2) that, for $0 \leq t \leq T_{1}$,

$$
\left\{\begin{aligned}
\left|\phi_{t}(t)\right|_{2} & \leq C\|v\|_{1}\left(\|\nabla \phi\|_{1}+|\phi|_{\infty}\right) \leq C c_{0} c_{2}, \\
\left|\phi_{t}(t)\right|_{D^{1}} & \leq C\|v\|_{2}\left(\|\nabla \phi\|_{1}+|\phi|_{\infty}\right) \leq C c_{0} c_{3}, \\
\left|\phi_{t}(t)\right|_{D^{2}} & \leq C\|v\|_{3}\left(\|\nabla \phi\|_{2}+|\phi|_{\infty}\right) \leq C c_{0} c_{4} .
\end{aligned}\right.
$$

At last, using the relation

$$
\phi_{t t}=-v_{t} \cdot \nabla \phi-v \cdot \nabla \phi_{t}-(\gamma-1) \phi_{t} \operatorname{div} v-(\gamma-1) \phi \operatorname{div} v_{t},
$$


and the assumption (3.24), one has, for $0 \leq t \leq T_{1}$, that

$$
\begin{gathered}
\left|\phi_{t t}(t)\right|_{2} \leq C\left(\left\|v_{t}\right\|_{1}\left(|\phi|_{\infty}+\|\nabla \phi\|_{2}\right)+\left\|\phi_{t}\right\|_{1}\|v\|_{2}\right) \leq C c_{4}^{3}, \\
\int_{0}^{t}\left\|\phi_{t t}\right\|_{1}^{2} \mathrm{~d} s \leq C \int_{0}^{t}\left(\left\|v_{t}\right\|_{2}^{2}\left(\|\nabla \phi\|_{2}+|\phi|_{\infty}\right)+\|v\|_{3}^{2}\left\|\phi_{t}\right\|_{2}^{2}\right) \mathrm{d} s \leq C c_{0}^{2} c_{4}^{2} .
\end{gathered}
$$

3.3.2. The a priori estimates for $\psi$. Next, we estimate $\psi$, which will be used to deal with the degenerate elliptic operator.

Lemma 3.3. Let $(\phi, u, h)$ be the unique classical solution to (3.15) in $[0, T] \times \mathbb{R}^{3}$. Then

$$
\begin{aligned}
& |\psi(t)|_{\infty}^{2}+\|\psi(t)\|_{D^{1} \cap D^{2}}^{2} \leq C c_{0}^{2}, \quad\left|\psi_{t}(t)\right|_{2} \leq C c_{3}^{2}, \quad\left|h_{t}(t)\right|_{\infty}^{2} \leq C c_{3}^{3} c_{4}, \\
& \left|\psi_{t}(t)\right|_{D^{1}}^{2}+\int_{0}^{t}\left(\left|\psi_{t t}\right|_{2}^{2}+\left|h_{t t}\right|_{6}^{2}\right) d s \leq C c_{4}^{4}, \quad \text { for } \quad 0 \leq t \leq T_{1} .
\end{aligned}
$$

Proof. Due to $\psi=\frac{a \delta}{\delta-1} \nabla h$ and the equation (3.15) $3, \psi$ satisfies the following system:

$$
\psi_{t}+\sum_{l=1}^{3} A_{l}(v) \partial_{l} \psi+B^{*}(v) \psi+a \delta(g \nabla \operatorname{div} v+\nabla g \operatorname{div} v)=0 .
$$

First, set $\varsigma=\left(\varsigma_{1}, \varsigma_{2}, \varsigma_{3}\right)^{\top}\left(1 \leq|\varsigma| \leq 2\right.$ and $\left.\varsigma_{i}=0,1,2\right)$. Applying $\partial_{x}^{\varsigma}$ to (3.30), multiplying by $2 \partial_{x}^{\varsigma} \psi$ and then integrating over $\mathbb{R}^{3}$, one can get

$$
\frac{d}{d t}\left|\partial_{x}^{\varsigma} \psi\right|_{2}^{2} \leq\left(\sum_{l=1}^{3}\left|\partial_{l} A_{l}\right|_{\infty}+\left|B^{*}\right|_{\infty}\right)\left|\partial_{x}^{\varsigma} \psi\right|_{2}^{2}+\left|\Theta_{\varsigma}\right|_{2}\left|\partial_{x}^{\varsigma} \psi\right|_{2}
$$

where

$$
\Theta_{\varsigma}=\partial_{x}^{\varsigma}\left(B^{*} \psi\right)-B^{*} \partial_{x}^{\varsigma} \psi+\sum_{l=1}^{3}\left(\partial_{x}^{\varsigma}\left(A_{l} \partial_{l} \psi\right)-A_{l} \partial_{l} \partial_{x}^{\varsigma} \psi\right)+a \delta \partial_{x}^{\varsigma}(g \nabla \operatorname{div} v+\nabla g \operatorname{div} v) .
$$

For $|\varsigma|=1$, it is easy to obtain

$$
\left|\Theta_{\varsigma}\right|_{2} \leq C\left(\left|\nabla^{2} v\right|_{2}\left(|\psi|_{\infty}+|\nabla g|_{\infty}\right)+|\nabla v|_{\infty}\left(|\nabla \psi|_{2}+\left|\nabla^{2} g\right|_{2}\right)+\left|g \nabla^{2} v\right|_{D^{1}}\right) .
$$

Similarly, for $|\varsigma|=2$, one has

$$
\begin{aligned}
\left|\Theta_{\varsigma}\right|_{2} \leq & C\left(|\nabla v|_{\infty}\left(\left|\nabla^{2} \psi\right|_{2}+\left|\nabla^{3} g\right|_{2}\right)+\left|\nabla^{2} v\right|_{3}\left(|\nabla \psi|_{6}+\left|\nabla^{2} g\right|_{6}\right)\right) \\
& +C\left|\nabla^{3} v\right|_{2}\left(|\psi|_{\infty}+|\nabla g|_{\infty}\right)+C|g \nabla \operatorname{div} v|_{D^{2}} .
\end{aligned}
$$

It follows from (3.31)-(3.33) and Gagliardo-Nirenberg inequality that

$$
\frac{d}{d t}\|\psi(t)\|_{D^{1} \cap D^{2}} \leq C c_{4}\|\psi(t)\|_{D^{1} \cap D^{2}}+C|g \nabla \operatorname{div} v|_{D^{2}}+C c_{4}^{2},
$$

which, along with the Gronwall's inequality, implies that for $0 \leq t \leq T_{1}$,

$$
\|\psi(t)\|_{D^{1} \cap D^{2}} \leq\left(c_{0}+C c_{4}^{2} t+C \int_{0}^{t}|g \nabla \operatorname{div} v|_{D^{2}} \mathrm{~d} s\right) \exp \left(C c_{4} t\right) \leq C c_{0} .
$$


Second, according to equations (3.30), for $0 \leq t \leq T_{1}$, it holds that

$$
\left\{\begin{array}{l}
\left|\psi_{t}(t)\right|_{2} \leq C\left(|v|_{\infty}|\psi|_{D^{1}}+|\nabla v|_{2}|\psi|_{\infty}+\left|g \nabla^{2} v\right|_{2}+|\nabla g|_{\infty}|\nabla v|_{2}\right) \leq C c_{3}^{2} \\
\left|\nabla \psi_{t}(t)\right|_{2} \leq C\left(\|v\|_{3}\left(\|\psi\|_{D^{1} \cap D^{2}}+\|\nabla g\|_{D^{1} \cap D^{2}}\right)+\left|g \nabla^{2} v\right|_{D^{1}}\right) \leq C c_{4}^{2}
\end{array}\right.
$$

Similarly, using

$$
\psi_{t t}=-\nabla(v \cdot \psi)_{t}-a \delta(g \nabla \operatorname{div} v+\nabla g \operatorname{div} v)_{t},
$$

for $0 \leq t \leq T_{1}$, one gets

$$
\begin{aligned}
\int_{0}^{t}\left|\psi_{t t}\right|_{2}^{2} \mathrm{~d} s \leq & C \int_{0}^{t}\left(\left|v_{t}\right|_{6}^{2}|\nabla \psi|_{3}^{2}+|\nabla v|_{\infty}^{2}\left|\psi_{t}\right|_{2}^{2}+|v|_{\infty}^{2}\left|\nabla \psi_{t}\right|_{2}^{2}+|\psi|_{\infty}^{2}\left|\nabla v_{t}\right|_{2}^{2}\right) \mathrm{d} s \\
& +\int_{0}^{t}\left(\left|(g \nabla \operatorname{div} v)_{t}\right|_{2}^{2}+|\nabla g|_{\infty}^{2}\left|\nabla v_{t}\right|_{2}^{2}+|\nabla v|_{\infty}^{2}\left|\nabla g_{t}\right|_{2}^{2}\right) \mathrm{d} s \leq C c_{4}^{4} .
\end{aligned}
$$

Finally, it follows from (2.2) and (3.24) that

$$
\begin{aligned}
|g \operatorname{div} v|_{\infty} \leq & C|g \operatorname{div} v|_{D^{1}}^{\frac{1}{2}}|g \operatorname{div} v|_{D^{2}}^{\frac{1}{2}} \leq C\left(|\nabla g|_{\infty}|\nabla v|_{2}+\left|g \nabla^{2} v\right|_{2}\right)^{\frac{1}{2}} \\
& \cdot\left(\left|\nabla^{2} g\right|_{2}|\nabla v|_{\infty}+|\nabla g|_{\infty}\left|\nabla^{2} v\right|_{2}+\left|g \nabla^{2} v\right|_{D^{1}}\right)^{\frac{1}{2}} \leq C c_{3}^{\frac{3}{2}} c_{4}^{\frac{1}{2}} .
\end{aligned}
$$

Then, together with (3.15) 3 , one gets easily that for $0 \leq t \leq T_{1}$,

$$
\begin{array}{r}
\left|h_{t}(t)\right|_{\infty} \leq C\left(|v|_{\infty}|\psi|_{\infty}+|g \operatorname{div} v|_{\infty}\right) \leq C c_{3}^{\frac{3}{2}} c_{4}^{\frac{1}{2}}, \\
\int_{0}^{t}\left|h_{t t}\right|_{6}^{2} \mathrm{~d} s \leq C \int_{0}^{t}\left(|v|_{\infty}\left|\psi_{t}\right|_{6}+\left|v_{t}\right|_{6}|\psi|_{\infty}+\left|g_{t}\right|_{\infty}|\nabla v|_{6}+\left|g \nabla v_{t}\right|_{6}\right)^{2} \mathrm{~d} s \leq C c_{4}^{4},
\end{array}
$$

where one has used the fact that

$$
\begin{aligned}
\left|g \nabla v_{t}\right|_{6} & \leq C\left(|\nabla g|_{\infty}\left|\nabla v_{t}\right|_{2}+\left|g \nabla^{2} v_{t}\right|_{2}\right) \\
& \leq C\left(|\nabla g|_{\infty}\left|\nabla v_{t}\right|_{2}+\left|\left(g \nabla^{2} v\right)_{t}\right|_{2}+\left|g_{t}\right|_{\infty}\left|\nabla^{2} v\right|_{2}\right) \leq C c_{4}^{2} .
\end{aligned}
$$

3.3.3. The a priori estimates for two h-related auxiliary variables. In order to obtain the uniformly a priori estimates and life span independent of the lower bound $\eta$ of $\phi_{0}$ for the solutions to the corresponding nonlinear problem, it is helpful to give some more precise estimates for another two new $h$-related quantities $\varphi$ and $f$ :

$$
\varphi=h^{-1}, \quad f=\psi \varphi=\frac{a \delta}{\delta-1} \nabla h / h=\left(f^{(1)}, f^{(2)}, f^{(3)}\right) .
$$

Lemma 3.4. Let $(\phi, u, h)$ be the unique classical solution to (3.15) in $[0, T] \times \mathbb{R}^{3}$. Then

$$
\begin{aligned}
\|\varphi(t)\|_{D^{1,6} \cap D^{2,3} \cap D^{3}}^{2}+\|f(t)\|_{L^{\infty} \cap L^{6} \cap D^{1,3} \cap D^{2}}^{2} & \leq C c_{0}^{4}, \\
h(t, x)> & \frac{1}{2 c_{0}}, \quad \frac{2}{3} \eta^{-2 e}<\varphi(t, x)<2\left|\varphi_{0}\right|_{\infty} \leq 2 c_{0}, \\
\left\|\varphi_{t}(t)\right\|_{L^{6} \cap D^{1,3} \cap D^{2}}^{2}+\left\|f_{t}(t)\right\|_{L^{3} \cap D^{1}}^{2} & \leq C c_{4}^{10},
\end{aligned}
$$

for $0 \leq t \leq T_{2}=\min \left\{T_{1},\left(1+C c_{4}\right)^{-4}\right\}$. 
Proof. Step 1: Estimates on $\varphi$. It is easy to see that $\varphi$ satisfies the following equation

$$
\varphi_{t}+v \cdot \nabla \varphi-(\delta-1) g \varphi^{2} \operatorname{div} v=0 .
$$

First, along with the particle path $X\left(t ; x_{0}\right)$ defined by

$$
\begin{cases}\frac{d}{d s} X\left(t ; x_{0}\right)=v\left(s, X\left(t ; x_{0}\right)\right), & 0 \leq t \leq T ; \\ X\left(0 ; x_{0}\right)=x_{0}, & x \in \mathbb{R}^{3},\end{cases}
$$

one has

$$
\varphi\left(t, X\left(t ; x_{0}\right)\right)=\varphi_{0}\left(x_{0}\right)\left(1+(1-\delta) \varphi_{0}\left(x_{0}\right) \int_{0}^{t} g \operatorname{div} v\left(s, X\left(s ; x_{0}\right)\right) \mathrm{d} s\right)^{-1},
$$

which, along with (3.40), implies that for $T_{2}=\min \left\{T_{1},\left(1+C c_{4}\right)^{-4}\right\}$,

$$
\frac{2}{3} \eta^{-2 e}<\varphi(t, x)<2\left|\varphi_{0}\right|_{\infty} \leq 2 c_{0}, \quad \text { for } \quad[t, x] \in\left[0, T_{2}\right] \times \mathbb{R}^{3} .
$$

Second, by the standard energy estimates for transport equations, one can obtain

$$
\begin{aligned}
\frac{d}{d t}|\nabla \varphi|_{6} \leq & C F(t)|\nabla \varphi|_{6}+C|\varphi|_{\infty}^{2}\left(\left|g \nabla^{2} v\right|_{6}+|\nabla v|_{\infty}|\nabla g|_{6}\right) \\
\frac{d}{d t}\left|\nabla^{2} \varphi\right|_{3} \leq & C F(t)\left|\nabla^{2} \varphi\right|_{3}+C|\nabla \varphi|_{6}\left(\left|\nabla^{2} v\right|_{6}+|\nabla \varphi|_{6}|g \operatorname{div} v|_{\infty}\right) \\
& +C|\varphi|_{\infty}^{2}\left(\left|g \nabla^{2} \operatorname{div} v\right|_{3}+\left|\nabla^{2} g\right|_{3}|\nabla v|_{\infty}+|\nabla g|_{\infty}\left|\nabla^{2} v\right|_{3}\right) \\
& +C|\varphi|_{\infty}|\nabla \varphi|_{6}\left(\left|g \nabla^{2} v\right|_{6}+|\nabla g|_{\infty}|\nabla v|_{6}\right), \\
\frac{d}{d t}\left|\nabla^{3} \varphi\right|_{2} \leq & C F(t)\left|\nabla^{3} \varphi\right|_{2}+C\left(|\nabla \varphi|_{6}\left|\nabla^{3} v\right|_{3}+\left|\nabla^{2} \varphi\right|_{3}\left|\nabla^{2} v\right|_{6}\right) \\
& +C|\varphi|_{\infty}^{2}\left(\left|g \nabla^{3} \operatorname{div} v\right|_{2}+|\nabla v|_{\infty}\left|\nabla^{3} g\right|_{2}+\left|\nabla^{2} g\right|_{6}\left|\nabla^{2} v\right|_{3}+|\nabla g|_{\infty}\left|\nabla^{3} v\right|_{2}\right) \\
& +\left.C\left|g \operatorname{div}_{\infty}\right| \nabla \varphi\right|_{6}\left|\nabla^{2} \varphi\right|_{3}+C|\nabla \varphi|_{6}^{2}\left(|\nabla g|_{\infty}|\nabla v|_{6}+\left|g \nabla^{2} v\right|_{6}\right) \\
& +C|\varphi|_{\infty}\left|\nabla^{2} \varphi\right|_{3}\left(|\nabla g|_{\infty}|\nabla v|_{6}+\left|g \nabla^{2} v\right|_{6}\right) \\
& +C|\varphi|_{\infty}|\nabla \varphi|_{6}\left(|\nabla g|_{\infty}\left|\nabla^{2} v\right|_{3}+\left|\nabla^{2} g\right|_{6}|\nabla v|_{6}+\left|g \nabla^{2} \operatorname{div} v\right|_{3}\right),
\end{aligned}
$$

where $F(t)=|\nabla v|_{\infty}+|\varphi|_{\infty}|g \operatorname{div} v|_{\infty}$. It follows from the Gronwall's inequality that

$$
|\varphi(t)|_{D^{1,6}}^{2}+|\varphi(t)|_{D^{2,3}}^{2}+|\varphi(t)|_{D^{3}}^{2} \leq C c_{0}^{2}, \quad \text { for } 0 \leq t \leq T_{2} .
$$

Finally, due to the equation (3.40), for $0 \leq t \leq T_{2}$, it holds that

$$
\begin{aligned}
\left|\varphi_{t}(t)\right|_{6} \leq & C\left(|v|_{\infty}|\nabla \varphi|_{6}+|\varphi|_{\infty}^{2}|g \operatorname{div} v|_{6}\right) \leq C c_{3}^{4}, \\
\left|\nabla \varphi_{t}(t)\right|_{3} \leq & C\left(|v|_{\infty}\left|\nabla^{2} \varphi\right|_{3}+|\nabla v|_{6}|\nabla \varphi|_{6}+\left.\left.|g \nabla v|_{6}|\nabla \varphi|_{6}\right|_{\varphi}\right|_{\infty}\right) \\
& +C|\varphi|_{\infty}^{2}\left(|\nabla g|_{\infty}|\nabla v|_{3}+\left|g \nabla^{2} v\right|_{3}\right) \leq C c_{3}^{4}, \\
\left|\nabla^{2} \varphi_{t}(t)\right|_{2} \leq & C\left(|v|_{\infty}\left|\nabla^{3} \varphi\right|_{2}+|\nabla v|_{6}\left|\nabla^{2} \varphi\right|_{3}+\left|\nabla^{2} v\right|_{3}|\nabla \varphi|_{6}\right) \\
& +C|\varphi|_{\infty}^{2}\left(|\nabla g|_{\infty}\left|\nabla^{2} v\right|_{2}+\left|g \nabla^{3} v\right|_{2}+|\nabla v|_{\infty}\left|\nabla^{2} g\right|_{2}\right) \\
& +C|\varphi|_{\infty}|\nabla \varphi|_{6}\left(\left|g \nabla^{2} v\right|_{3}+|\nabla g|_{\infty}|\nabla v|_{3}\right) \\
& +C|g \nabla v|_{6}\left(|\nabla \varphi|_{6}^{2}+|\varphi|_{\infty}\left|\nabla^{2} \varphi\right|_{3}\right) \leq C c_{4}^{4} .
\end{aligned}
$$


Step 2: Estimates on $f$. Based on the estimates obtained in Lemma 3.3 and the above step, due to $f=\psi \varphi$, it is easy to check that for $0 \leq t \leq T_{2}$,

$$
\begin{aligned}
|f(t)|_{\infty} & \leq C c_{0}^{2}, \quad|f(t)|_{6} \leq|\psi|_{6}|\varphi|_{\infty} \leq C c_{0}^{2}, \\
|\nabla f(t)|_{3} & \leq C\left(|\nabla \varphi|_{6}|\psi|_{6}+|\varphi|_{\infty}|\nabla \psi|_{3}\right) \leq C c_{0}^{2}, \\
\left|\nabla^{2} f(t)\right|_{2} & \leq C\left(|\varphi|_{\infty}\left|\nabla^{2} \psi\right|_{2}+|\psi|_{6}\left|\nabla^{2} \varphi\right|_{3}+|\nabla \varphi|_{6}|\nabla \psi|_{3}\right) \leq C c_{0}^{2}, \\
\left|f_{t}(t)\right|_{3} & \leq C\left(|\psi|_{6}\left|\varphi_{t}\right|_{6}+|\varphi|_{\infty}\left|\psi_{t}\right|_{3}\right)(t) \leq C c_{4}^{5}, \\
\left|\nabla f_{t}(t)\right|_{2} & \leq C\left(|\psi|_{6}\left|\nabla \varphi_{t}\right|_{3}+|\varphi|_{\infty}\left|\nabla \psi_{t}\right|_{2}+|\nabla \psi|_{3}\left|\varphi_{t}\right|_{6}+|\nabla \varphi|_{6}\left|\psi_{t}\right|_{3}\right)(t) \leq C c_{4}^{5} .
\end{aligned}
$$

It follows from the definition that $f$ satisfies the following equations:

$$
f_{t}+\sum_{l=1}^{3} A_{l}(v) \partial_{l} f+B^{*}(v) f+a \delta(g \varphi \nabla \operatorname{div} v+\varphi \nabla g \operatorname{div} v)-(\delta-1) g \varphi f \operatorname{div} v=0
$$

3.3.4. The a priori estimates for $u$. Based on the estimates of $\phi$ and $h$ obtained in Lemmas 3.2 3.4, we are now ready to give the lower order estimates for the velocity $u$ as follows.

Lemma 3.5. Let $(\phi, u, h)$ be the unique classical solution to (3.15) in $[0, T] \times \mathbb{R}^{3}$. Then

$$
\begin{gathered}
|\sqrt{h} \nabla u(t)|_{2}^{2}+\|u(t)\|_{1}^{2}+\int_{0}^{t}\left(\|\nabla u\|_{1}^{2}+\left|u_{t}\right|_{2}^{2}\right) d s \leq C c_{0}^{4}, \\
\left(|u|_{D^{2}}^{2}+\left|h \nabla^{2} u\right|_{2}^{2}+\left|u_{t}\right|_{2}^{2}\right)(t)+\int_{0}^{t}\left(|u|_{D^{3}}^{2}+\left|u_{t}\right|_{D^{1}}^{2}\right) d s \leq C c_{2}^{9} c_{3},
\end{gathered}
$$

for $0 \leq t \leq T_{3}=\min \left(T_{2},\left(1+c_{4}\right)^{-14}\right)$.

Proof. Step 1: Estimate on $|u|_{2}$. Multiplying $(\sqrt{3.15})_{2}$ by $u$ and integrating over $\mathbb{R}^{3}$, one gets from Gagliardo-Nirenberg inequality, Hölder's inequality and Young's inequality that

$$
\begin{aligned}
& \frac{1}{2} \frac{d}{d t}|u|_{2}^{2}+a \alpha\left|\left(h^{2}+\epsilon^{2}\right)^{\frac{1}{4}} \nabla u\right|_{2}^{2}+a(\alpha+\beta)\left|\left(h^{2}+\epsilon^{2}\right)^{\frac{1}{4}} \operatorname{div} u\right|_{2}^{2} \\
= & -\int\left(v \cdot \nabla v+\nabla \phi+a \nabla \sqrt{h^{2}+\epsilon^{2}} \cdot Q(u)-\psi \cdot Q(v)\right) \cdot u \\
\leq & C\left(|v|_{\infty}|\nabla v|_{2}+|\nabla \phi|_{2}+|\psi|_{\infty}|\sqrt{h} \nabla u|_{2}|\varphi|_{\infty}^{\frac{1}{2}}+|\psi|_{\infty}|\nabla v|_{2}\right)|u|_{2} \\
\leq & C c_{3}^{3}|u|_{2}^{2}+C c_{3}+\frac{1}{2} a \alpha|\sqrt{h} \nabla u|_{2}^{2},
\end{aligned}
$$

which, along with the Gronwall's inequality, implies immediately that for $0 \leq t \leq T_{2}$,

$$
|u(t)|_{2}^{2}+\frac{a \alpha}{2} \int_{0}^{t}|\sqrt{h} \nabla u|_{2}^{2} \mathrm{~d} s \leq C\left(\left|u_{0}\right|_{2}^{2}+c_{3} t\right) \exp \left(C c_{3}^{3} t\right) \leq C c_{0}^{2} .
$$


Step 2: Estimate on $|\nabla u|_{2}$. Multiplying $(\underline{3.15})_{2}$ by $u_{t}$ and integrating over $\mathbb{R}^{3}$, one gets from the Gagliardo-Nirenberg, Hölder's and Young's inequalities that

$$
\begin{aligned}
& \frac{1}{2} \frac{d}{d t}\left(a \alpha\left|\left(h^{2}+\epsilon^{2}\right)^{\frac{1}{4}} \nabla u\right|_{2}^{2}+a(\alpha+\beta)\left|\left(h^{2}+\epsilon^{2}\right)^{\frac{1}{4}} \operatorname{div} u\right|_{2}^{2}\right)+\left|u_{t}\right|_{2}^{2} \\
= & -\int\left(v \cdot \nabla v+\nabla \phi+a \nabla \sqrt{h^{2}+\epsilon^{2}} \cdot Q(u)+\psi \cdot Q(v)\right) \cdot u_{t} \\
& -\int a \frac{h}{\sqrt{h^{2}+\epsilon^{2}}} h_{t}\left(\alpha|\nabla u|^{2}+(\alpha+\beta)|\operatorname{div} u|^{2}\right) \\
\leq & C\left(|v|_{\infty}|\nabla v|_{2}+|\nabla \phi|_{2}+|\psi|_{\infty}|\sqrt{h} \nabla u|_{2}|\varphi|_{\infty}^{\frac{1}{2}}+|\psi|_{\infty}|\nabla v|_{2}\right)\left|u_{t}\right|_{2} \\
& +C\left|h_{t}\right|_{\infty}|\varphi|_{\infty}|\sqrt{h} \nabla u|_{2}^{2} \leq C c_{4}^{3}|\sqrt{h} \nabla u|_{2}^{2}+C c_{3}^{4}+\frac{1}{2}\left|u_{t}\right|_{2}^{2},
\end{aligned}
$$

which, along with the Gronwall's inequality, implies that for $0 \leq t \leq T_{2}$,

$$
|\sqrt{h} \nabla u(t)|_{2}^{2}+\int_{0}^{t}\left|u_{t}\right|_{2}^{2} \mathrm{~d} s \leq C\left(c_{0}^{2}+c_{3}^{4} t\right) \exp \left(C c_{4}^{3} t\right) \leq C c_{0}^{2}, \text { and }|u(t)|_{D^{1}} \leq C c_{0}^{\frac{3}{2}} .
$$

It follows from the definitions of the Lamé operator $L$ and $\psi$ that

$$
\begin{aligned}
a L\left(\sqrt{h^{2}+\epsilon^{2}} u\right) & =a \sqrt{h^{2}+\epsilon^{2}} L u-G\left(\nabla \sqrt{h^{2}+\epsilon^{2}}, u\right) \\
& =-u_{t}-v \cdot \nabla v-\nabla \phi+\psi \cdot Q(v)-G\left(\nabla \sqrt{h^{2}+\epsilon^{2}}, u\right) .
\end{aligned}
$$

Then Lemma 2.5 implies that

$$
\begin{aligned}
\left|\sqrt{h^{2}+\epsilon^{2}} u(t)\right|_{D^{2}} & \leq C\left(\left|u_{t}+v \cdot \nabla v+\nabla \phi-\psi \cdot Q(v)\right|_{2}+\left|G\left(\nabla \sqrt{h^{2}+\epsilon^{2}}, u\right)\right|_{2}\right) \\
& \leq C\left(\left|u_{t}\right|_{2}+c_{2}^{\frac{7}{2}} c_{3}^{\frac{1}{2}}\right) \\
\left|\sqrt{h^{2}+\epsilon^{2}} \nabla^{2} u(t)\right|_{2} & \leq C\left(\left|\sqrt{h^{2}+\epsilon^{2}} u\right|_{D^{2}}+|\nabla \psi|_{3}|u|_{6}+\left.|\psi|_{\infty}|\nabla u|_{2}|+| \psi\right|_{\infty} ^{2}|u|_{2}|\varphi|_{\infty}\right) \\
& \leq C\left(\left|\sqrt{h^{2}+\epsilon^{2}} u\right|_{D^{2}}+c_{0}^{4}\right)
\end{aligned}
$$

for $0 \leq t \leq T_{2}$, where one has used the fact that

$$
|v \cdot \nabla v|_{2} \leq C|v|_{6}|\nabla v|_{3} \leq C|\nabla v|_{2}^{\frac{3}{2}}\left|\nabla^{2} v\right|_{2}^{\frac{1}{2}}, \quad|\nabla \psi|_{3}|u|_{6} \leq C c_{0}|\nabla u|_{2} \leq C c_{0}^{\frac{5}{2}} .
$$

According to (3.50) and (3.52), one gets

$$
\int_{0}^{t}\left(\left|h \nabla^{2} u\right|_{2}^{2}+\left|\nabla^{2} u\right|_{2}^{2}\right) \mathrm{d} s \leq C c_{0}^{4}, \quad \text { for } \quad 0 \leq t \leq T^{\prime}=\min \left(T_{2},\left(1+c_{4}\right)^{-8}\right) .
$$

Step 3: Estimate on $|u|_{D^{2}}$. First, applying $\partial_{t}$ to $(3.15)_{2}$ yields

$$
u_{t t}+a \sqrt{h^{2}+\epsilon^{2}} L u_{t}=-(v \cdot \nabla v)_{t}-\nabla \phi_{t}-\frac{a h}{\sqrt{h^{2}+\epsilon^{2}}} h_{t} L u+(\psi \cdot Q(v))_{t} .
$$


Second, multiplying (3.53) by $u_{t}$ and integrating over $\mathbb{R}^{3}$ lead to

$$
\begin{aligned}
& \frac{1}{2} \frac{d}{d t}\left|u_{t}\right|_{2}^{2}+a \alpha\left|\left(h^{2}+\epsilon^{2}\right)^{\frac{1}{4}} \nabla u_{t}\right|_{2}^{2}+a(\alpha+\beta)\left|\left(h^{2}+\epsilon^{2}\right)^{\frac{1}{4}} \operatorname{div} u_{t}\right|_{2}^{2} \\
= & \int\left(-(v \cdot \nabla v)_{t}-\nabla \phi_{t}-a \nabla \sqrt{h^{2}+\epsilon^{2}} \cdot Q(u)_{t}\right. \\
& \left.-\frac{a h}{\sqrt{h^{2}+\epsilon^{2}}} h_{t} L u+(\psi \cdot Q(v))_{t}\right) \cdot u_{t} \\
\leq & C\left(|v|_{\infty}\left|\nabla v_{t}\right|_{2}+\left|v_{t}\right|_{2}|\nabla v|_{\infty}+\left|\nabla \phi_{t}\right|_{2}+|\psi|_{\infty}\left|\sqrt{h} \nabla u_{t}\right|_{2}|\varphi|_{\infty}^{\frac{1}{2}}\right)\left|u_{t}\right|_{2} \\
& +C\left(\left|h_{t}\right|_{\infty}\left|\nabla^{2} u\right|_{2}+|\psi|_{\infty}\left|\nabla v_{t}\right|_{2}+\left|\psi_{t}\right|_{2}|\nabla v|_{\infty}\right)\left|u_{t}\right|_{2} .
\end{aligned}
$$

Integrating (3.54) over $(\tau, t)(\tau \in(0, t))$ and using Young's inequality, one has

$$
\begin{aligned}
& \frac{1}{2}\left|u_{t}(t)\right|_{2}^{2}+\frac{a \alpha}{2} \int_{\tau}^{t}\left|\sqrt{h} \nabla u_{t}(s)\right|_{2}^{2} \mathrm{~d} s \\
\leq & \frac{1}{2}\left|u_{t}(\tau)\right|_{2}^{2}+C c_{4}^{4} \int_{0}^{t}\left|u_{t}(s)\right|_{2}^{2} \mathrm{~d} s+C c_{4}^{2} t+C c_{0}^{4}, \quad \text { for } \quad 0 \leq t \leq T^{\prime} .
\end{aligned}
$$

It follows from the momentum equations $(3.15))_{2}$ that

$$
\left|u_{t}(\tau)\right|_{2} \leq C\left(|v|_{\infty}|\nabla v|_{2}+|\nabla \phi|_{2}+|(h+\epsilon) L u|_{2}+|\psi|_{\infty}|\nabla v|_{2}\right)(\tau),
$$

which, along with the assumption (3.16), Lemma 3.1 and (3.19)-(3.20), implies that

$$
\begin{aligned}
\limsup _{\tau \rightarrow 0}\left|u_{t}(\tau)\right|_{2} & \leq C\left(\left|v_{0}\right|_{\infty}\left|\nabla v_{0}\right|_{2}+\left|\nabla \phi_{0}\right|_{2}+\left|g_{2}\right|_{2}+\left|L u_{0}\right|_{2}+\left|\psi_{0}\right|_{\infty}\left|\nabla v_{0}\right|_{2}\right) \\
& \leq C c_{0}^{2} .
\end{aligned}
$$

Letting $\tau \rightarrow 0$ in (3.55), one gets from the Gronwall's inequality that for $0 \leq t \leq T^{\prime}$,

$$
\left|u_{t}(t)\right|_{2}^{2}+\int_{0}^{t}\left|\sqrt{h} \nabla u_{t}\right|_{2}^{2} \mathrm{~d} s \leq C\left(c_{4}^{2} t+c_{0}^{4}\right) \exp \left(C c_{4}^{4} t\right) \leq C c_{0}^{4}, \quad \int_{0}^{t}\left|\nabla u_{t}\right|_{2}^{2} \mathrm{~d} s \leq C c_{0}^{5} .
$$

It follows from (3.52) that for $0 \leq t \leq T^{\prime}$,

$$
\left|\sqrt{h^{2}+\epsilon^{2}} u(t)\right|_{D^{2}} \leq C c_{2}^{\frac{7}{2}} c_{3}^{\frac{1}{2}}, \quad\left|h \nabla^{2} u(t)\right|_{2} \leq C c_{2}^{\frac{7}{2}} c_{3}^{\frac{1}{2}}, \quad|u(t)|_{D^{2}} \leq C c_{2}^{\frac{9}{2}} c_{3}^{\frac{1}{2}} .
$$

By the classical estimates for elliptic systems in Lemma 2.5 and (3.51), one gets

$$
\begin{aligned}
\left|\sqrt{h^{2}+\epsilon^{2}} u(t)\right|_{D^{3}} \leq & C\left(\left|u_{t}+v \cdot \nabla v+\nabla \phi-\psi \cdot Q(v)\right|_{D^{1}}+\left|G\left(\nabla \sqrt{h^{2}+\epsilon^{2}}, u\right)\right|_{D^{1}}\right) \\
\leq & C\left(\left|u_{t}\right|_{D^{1}}+c_{3}^{6}\right) \\
\left|\sqrt{h^{2}+\epsilon^{2}} \nabla^{3} u(t)\right|_{2} \leq & C\left(\left|\sqrt{h^{2}+\epsilon^{2}} u(t)\right|_{D^{3}}+\|u\|_{2}\|\psi\|_{D^{1} \cap D^{2}}\right) \\
& +C\|u\|_{1}\left(1+\|\psi\|_{D^{1} \cap D^{2}}^{3}\right)\left(1+|\varphi|_{\infty}^{2}\right) \\
\leq & C\left(\left|\sqrt{h^{2}+\epsilon^{2}} u(t)\right|_{D^{3}}+c_{3}^{7}\right)
\end{aligned}
$$

which, along with (3.58)-(3.59), implies that,

$$
\int_{0}^{t}\left(\left|h \nabla^{3} u\right|_{2}^{2}+\left|h \nabla^{2} u\right|_{D^{1}}^{2}+|u|_{D^{3}}^{2}\right) \mathrm{d} s \leq C c_{0}^{7},
$$

for $0 \leq t \leq T_{3}=\min \left(T^{\prime},\left(1+c_{4}\right)^{-14}\right)=\min \left(T_{2},\left(1+c_{4}\right)^{-14}\right)$. 
Next some estimates on the higher order derivatives of the velocity $u$ are established in the following two lemmas.

Lemma 3.6. Let $(\phi, u, h)$ be the unique classical solution to (3.15) in $[0, T] \times \mathbb{R}^{3}$. Then,

$$
\begin{array}{r}
\left(\left|\sqrt{h} \nabla u_{t}\right|_{2}^{2}+\left|u_{t}\right|_{D^{1}}^{2}+|u|_{D^{3}}^{2}+\left|h \nabla^{2} u\right|_{D^{1}}^{2}\right)(t)+\int_{0}^{t}\left|u_{t}\right|_{D^{2}}^{2} d s \leq C c_{3}^{16}, \\
\int_{0}^{t}\left(\left|u_{t t}\right|_{2}^{2}+|u|_{D^{4}}^{2}+\left|h \nabla^{2} u\right|_{D^{2}}^{2}+\left|\left(h \nabla^{2} u\right)_{t}\right|_{2}^{2}\right) d s \leq C c_{0}^{11},
\end{array}
$$

for $0 \leq t \leq T_{4}=\min \left(T_{3},\left(1+c_{4}\right)^{-20}\right)$.

Proof. Multiplying (3.53) by $u_{t t}$ and integrating over $\mathbb{R}^{3}$ give

$$
\begin{aligned}
& \frac{1}{2} \frac{d}{d t}\left(a \alpha\left|\left(h^{2}+\epsilon^{2}\right)^{\frac{1}{4}} \nabla u_{t}\right|_{2}^{2}+a(\alpha+\beta)\left|\left(h^{2}+\epsilon^{2}\right)^{\frac{1}{4}} \operatorname{div} u_{t}\right|_{2}^{2}\right)+\left|u_{t t}\right|_{2}^{2} \\
= & \int\left(-(v \cdot \nabla v)_{t}-\nabla \phi_{t}-\frac{a h}{\sqrt{h^{2}+\epsilon^{2}}} h_{t} L u+\frac{a h}{\sqrt{h^{2}+\epsilon^{2}}} \nabla h \cdot Q\left(u_{t}\right)\right) \cdot u_{t t} \\
& +\int\left(\frac{a h}{\sqrt{h^{2}+\epsilon^{2}}} h_{t}\left(\alpha\left|\nabla u_{t}\right|^{2}+(\alpha+\beta)\left|\operatorname{div} u_{t}\right|^{2}\right)+(\psi \cdot Q(v))_{t} \cdot u_{t t}\right) \\
\leq & C\left(\left|v_{t}\right|_{2}|\nabla v|_{\infty}+|v|_{\infty}\left|\nabla v_{t}\right|_{2}+\left|\nabla \phi_{t}\right|_{2}+\left|h_{t}\right|_{\infty}\left|\nabla^{2} u\right|_{2}+\left|\psi_{t}\right|_{2}|\nabla v|_{\infty}\right)\left|u_{t t}\right|_{2} \\
& +C\left(|\psi|_{\infty}\left|\nabla v_{t}\right|_{2}+|\psi|_{\infty}|\varphi|_{\infty}^{\frac{1}{2}}\left|\sqrt{h} \nabla u_{t}\right|_{2}\right)\left|u_{t t}\right|_{2}+C\left|h_{t}\right|_{\infty}|\varphi|_{\infty}\left|\sqrt{h} \nabla u_{t}\right|_{2}^{2} .
\end{aligned}
$$

Integrating (3.62) over $(\tau, t)$ shows that for $0 \leq t \leq T_{3}$,

$$
\left|\sqrt{h} \nabla u_{t}(t)\right|_{2}^{2}+\int_{\tau}^{t}\left|u_{t t}\right|_{2}^{2} \mathrm{~d} s \leq C\left|\left(h^{2}+\epsilon^{2}\right)^{\frac{1}{4}} \nabla u_{t}(\tau)\right|_{2}^{2}+C c_{4}^{14} t+C c_{4}^{3} \int_{0}^{t}\left|\sqrt{h} \nabla u_{t}\right|_{2}^{2} \mathrm{~d} s .
$$

On the other hand, it follows from the momentum equations $(3.15)_{2}$ that

$$
\left|\sqrt{h} \nabla u_{t}(\tau)\right|_{2} \leq\left(\left|\sqrt{h} \nabla\left(v \cdot \nabla v+\nabla \phi+a \sqrt{h^{2}+\epsilon^{2}} L u-\psi \cdot Q(v)\right)\right|_{2}\right)(\tau) .
$$

Then by the assumption (3.16), Lemma 3.1, (3.19) $-(3.20)$ and (3.22) $-(3.23)$, one has

$$
\begin{aligned}
\limsup _{\tau \rightarrow 0}\left|\sqrt{h} \nabla u_{t}(\tau)\right|_{2} \leq & C\left(\left|\sqrt{h}_{0} \nabla\left(u_{0} \cdot \nabla u_{0}\right)\right|_{2}+\left|\sqrt{h_{0}} \nabla^{2} \phi_{0}\right|_{2}\right. \\
& \left.+\left|\sqrt{h_{0}} \nabla\left(\psi_{0} \cdot Q\left(u_{0}\right)\right)\right|_{2}+\left|g_{3}\right|_{2}+\epsilon\left|\nabla L u_{0}\right|_{2}\right) \\
\leq & C\left(\left|\phi_{0}^{e} u_{0}\right|_{6}\left|\nabla^{2} u_{0}\right|_{3}+\left.\left|\nabla u_{0}\right|_{\infty}\left|\phi_{0}^{e} \nabla u_{0}\right|_{2}|+| \phi_{0}^{e} \nabla^{2} \phi_{0}\right|_{2}\right) \\
+ & C\left(\left|\phi_{0}^{e} \nabla^{2} u_{0}\right|_{2}\left|\psi_{0}\right|_{\infty}+\left|\nabla \psi_{0}\right|_{3}\left|\phi_{0}^{e} \nabla u_{0}\right|_{6}+c_{0}\right) \leq C c_{0}^{3},
\end{aligned}
$$

which implies that

$$
\lim \sup _{\tau \rightarrow 0}\left|\sqrt{\epsilon} \nabla u_{t}(\tau)\right|_{2} \leq \lim \sup _{\tau \rightarrow 0} \sqrt{\epsilon}|\varphi|_{\infty}^{\frac{1}{2}}\left|\sqrt{h} \nabla u_{t}(\tau)\right|_{2} \leq C c_{0}^{\frac{7}{2}} .
$$

Letting $\tau \rightarrow 0$ in (3.63) and using the Gronwall's inequality, one can obtain

$$
\left|\sqrt{h} \nabla u_{t}(t)\right|_{2}^{2}+\int_{0}^{t}\left|u_{t t}(s)\right|_{2}^{2} \mathrm{~d} s \leq C\left(c_{0}^{7}+c_{4}^{14} t\right) \exp \left(C c_{4}^{3} t\right) \leq C c_{0}^{7}, \quad\left|\nabla u_{t}(t)\right|_{2}^{2} \leq C c_{0}^{8},
$$


for $0 \leq t \leq T_{3}$, which, along with (3.60), implies that

$$
\left|\sqrt{h^{2}+\epsilon^{2}} u\right|_{D^{3}}+\left|\sqrt{h^{2}+\epsilon^{2}} \nabla^{3} u\right|_{2}+\left|h \nabla^{2} u\right|_{D^{1}} \leq C c_{3}^{7}, \quad\left|\nabla^{3} u\right|_{2} \leq C c_{3}^{8} .
$$

It follows from (3.53) that

$$
\begin{aligned}
a \sqrt{h^{2}+\epsilon^{2}} L u_{t} & =-u_{t t}-(v \cdot \nabla v)_{t}-\nabla \phi_{t}-\frac{a h}{\sqrt{h^{2}+\epsilon^{2}}} h_{t} L u+(\psi \cdot Q(v))_{t} \\
& =a L\left(\sqrt{h^{2}+\epsilon^{2}} u_{t}\right)+G\left(\nabla \sqrt{h^{2}+\epsilon^{2}}, u_{t}\right) .
\end{aligned}
$$

Now applying Lemma 2.5 to (3.51) and (3.67), one gets for $0 \leq t \leq T_{3}$,

$$
\begin{aligned}
\left|\sqrt{h^{2}+\epsilon^{2}} u_{t}(t)\right|_{D^{2}} \leq & C\left|u_{t t}+(v \cdot \nabla v)_{t}+\nabla \phi_{t}-(\psi \cdot Q(v))_{t}+\frac{a h}{\sqrt{h^{2}+\epsilon^{2}}} h_{t} L u\right|_{2} \\
& +C\left|G\left(\nabla \sqrt{h^{2}+\epsilon^{2}}, u_{t}\right)\right|_{2} \leq C\left(\left|u_{t t}(t)\right|_{2}+c_{4}^{7}\right), \\
\left|\sqrt{h^{2}+\epsilon^{2}} \nabla^{2} u_{t}(t)\right|_{2} \leq & C\left(\left|\sqrt{h^{2}+\epsilon^{2}} u_{t}(t)\right|_{D^{2}}+\|\psi\|_{D^{1} \cap D^{2}}\left|\nabla u_{t}\right|_{2}+|\psi|_{\infty}^{2}\left|u_{t}\right|_{2}|\varphi|_{\infty}\right) \\
\leq & C\left(\left|\sqrt{h^{2}+\epsilon^{2}} u_{t}\right|_{D^{2}}+c_{4}^{8}\right), \\
\left|\left(h \nabla^{2} u\right)_{t}(t)\right|_{2} \leq & C\left(\left|h \nabla^{2} u_{t}\right|_{2}+\left|h_{t}\right|_{\infty}\left|\nabla^{2} u\right|_{2}\right) \leq C\left(\left|u_{t t}(t)\right|_{2}+c_{4}^{8}\right), \\
|u(t)|_{D^{4}} \leq & C\left|\left(h^{2}+\epsilon^{2}\right)^{-\frac{1}{2}}\left(u_{t}+v \cdot \nabla v+\nabla \phi-\psi \cdot Q(v)\right)\right|_{D^{2}} \\
\leq & C\left(c_{0}^{2}\left|u_{t t}(t)\right|_{2}+c_{4}^{10}\right) .
\end{aligned}
$$

Using the momentum equations $(\underline{3.15})_{2}$, for multi-index $\xi \in R^{3}$ with $|\xi|=2$, one has

$$
\begin{aligned}
a L\left(\sqrt{h^{2}+\epsilon^{2}} \nabla^{\xi} u\right)= & a \sqrt{h^{2}+\epsilon^{2}} \nabla^{\xi} L u-G\left(\nabla \sqrt{h^{2}+\epsilon^{2}}, \nabla^{\xi} u\right) \\
= & -\sqrt{h^{2}+\epsilon^{2}} \nabla^{\xi}\left(\left(h^{2}+\epsilon^{2}\right)^{-\frac{1}{2}}\left(u_{t}+v \cdot \nabla v+\nabla \phi-\psi \cdot Q(v)\right)\right) \\
& -G\left(\nabla \sqrt{h^{2}+\epsilon^{2}}, \nabla^{\xi} u\right),
\end{aligned}
$$

which implies that

$$
\begin{aligned}
& \left|\sqrt{h^{2}+\epsilon^{2}} \nabla^{2} u(t)\right|_{D^{2}} \\
\leq & C\left|\sqrt{h^{2}+\epsilon^{2}} \nabla^{\xi}\left(\left(h^{2}+\epsilon^{2}\right)^{-\frac{1}{2}}\left(u_{t}+v \cdot \nabla v+\nabla \phi-\psi \cdot Q(v)\right)\right)\right|_{2} \\
& +C\left(|\psi|_{\infty}|u|_{D^{3}}+|\nabla \psi|_{3}\left|\nabla^{2} u\right|_{6}+\left|\nabla^{2} u\right|_{2}|\psi|_{\infty}^{2}|\varphi|_{\infty}\right) \\
\leq & C\left(\left|u_{t}(t)\right|_{D^{2}}+c_{4}^{9}\right) .
\end{aligned}
$$

Thus, for $T_{4}=\min \left(T_{3},\left(1+c_{4}\right)^{-20}\right)$, it holds that

$$
\int_{0}^{T_{4}}\left(\left|h \nabla^{2} u_{t}\right|_{2}^{2}+\left|u_{t}\right|_{D^{2}}^{2}+|u|_{D^{4}}^{2}+\left|h \nabla^{2} u\right|_{D^{2}}^{2}+\left|\left(h \nabla^{2} u\right)_{t}\right|_{2}^{2}\right) \mathrm{d} t \leq C c_{0}^{11} .
$$

Lemma 3.7. Let $(\phi, u, h)$ be the unique classical solution to (3.15) in $[0, T] \times \mathbb{R}^{3}$. Then,

$$
t\left(\left|u_{t}(t)\right|_{D^{2}}^{2}+\left|u_{t t}(t)\right|_{2}^{2}+|u(t)|_{D^{4}}^{2}\right)+\int_{0}^{t} s\left(\left|u_{t t}\right|_{D^{1}}^{2}+\left|u_{t}\right|_{D^{3}}^{2}\right) d s \leq C c_{4}^{8},
$$

for $0 \leq t \leq T_{5}=\min \left(T_{4},\left(1+c_{5}\right)^{-14}\right)$. 
Proof. Now applying $\partial_{t}$ to 3.53 yields

$$
\begin{gathered}
u_{t t t}+a \sqrt{h^{2}+\epsilon^{2}} L u_{t t}=-2 v_{t} \cdot \nabla v_{t}-v_{t t} \cdot \nabla v-v \cdot \nabla v_{t t}-\nabla \phi_{t t}-\frac{a \epsilon^{2} h_{t}^{2}}{\left(h^{2}+\epsilon^{2}\right)^{\frac{3}{2}}} L u \\
-\frac{a h}{\sqrt{h^{2}+\epsilon^{2}}} h_{t t} L u-2 \frac{a h}{\sqrt{h^{2}+\epsilon^{2}}} h_{t} L u_{t}+2 \psi_{t} \cdot Q\left(v_{t}\right)+\psi_{t t} \cdot Q(v)+\psi \cdot Q\left(v_{t t}\right) .
\end{gathered}
$$

Multiplying (3.72) by $u_{t t}$ and integrating over $\mathbb{R}^{3}$ give

$$
\begin{aligned}
& \frac{1}{2} \frac{d}{d t}\left|u_{t t}\right|_{2}^{2}+a \alpha\left|\left(h^{2}+\epsilon^{2}\right)^{\frac{1}{4}} \nabla u_{t t}\right|_{2}^{2}+a(\alpha+\beta)\left|\left(h^{2}+\epsilon^{2}\right)^{\frac{1}{4}} \operatorname{div} u_{t t}\right|_{2}^{2} \\
= & \int\left(-2 v_{t} \cdot \nabla v_{t}-v_{t t} \cdot \nabla v-v \cdot \nabla v_{t t}-\nabla \phi_{t t}-\frac{a h}{\sqrt{h^{2}+\epsilon^{2}}} \nabla h \cdot Q(u)_{t t}\right) \cdot u_{t t} \\
& -\int\left(\frac{a \epsilon^{2} h_{t}^{2}}{\left(h^{2}+\epsilon^{2}\right)^{\frac{3}{2}}} L u+\frac{a h}{\sqrt{h^{2}+\epsilon^{2}}} h_{t t} L u\right) \cdot u_{t t} \\
& +\int\left(-2 \frac{a h}{\sqrt{h^{2}+\epsilon^{2}}} h_{t} L u_{t}+2 \psi_{t} \cdot Q\left(v_{t}\right)+\psi_{t t} \cdot Q(v)+\psi \cdot Q\left(v_{t t}\right)\right) \cdot u_{t t} \\
\leq & C\left(\left|\nabla v_{t}\right|_{6}\left|v_{t}\right|_{3}+|\nabla v|_{\infty}\left|v_{t t}\right|_{2}+|v|_{\infty}\left|\nabla v_{t t}\right|_{2}+\left|h_{t t}\right|_{6}\left|\nabla^{2} u\right|_{3}\right)\left|u_{t t}\right|_{2} \\
& +C\left(\left|\phi_{t t}\right|_{2}+|\psi|_{\infty}\left|u_{t t}\right|_{2}\right)\left|\sqrt{h} \nabla u_{t t}\right|_{2}|\varphi|_{\infty}^{\frac{1}{2}}+C\left|h_{t}\right|_{\infty}^{2}|\varphi|_{\infty}\left|\nabla^{2} u\right|_{2}\left|u_{t t}\right|_{2} \\
& +C\left|h_{t}\right|_{\infty}\left|\nabla^{2} u_{t}\right|_{2}\left|u_{t t}\right|_{2}+C\left(\left|\psi_{t}\right|_{3}\left|\nabla v_{t}\right|_{6}+\left|\psi_{t t}\right|_{2}|\nabla v|_{\infty}+|\psi|_{\infty}\left|\nabla v_{t t}\right|_{2}\right)\left|u_{t t}\right|_{2} .
\end{aligned}
$$

Multiplying both sides of (3.73) by $t$ and integrating over $(\tau, t)$, one can get

$$
t\left|u_{t t}(t)\right|_{2}^{2}+\frac{a \alpha}{2} \int_{\tau}^{t} s\left|\sqrt{h} \nabla u_{t t}\right|_{2}^{2} \mathrm{~d} s \leq \tau\left|u_{t t}(\tau)\right|_{2}^{2}+C c_{4}^{4}+C c_{5}^{3} \int_{\tau}^{t} s\left|u_{t t}\right|_{2}^{2} \mathrm{~d} s
$$

where $0 \leq t \leq T_{5}=\min \left(T_{4},\left(1+c_{5}\right)^{-20}\right)$.

It follows from (3.66) and Lemma 2.4 that there exists a sequence $s_{k}$ such that

$$
s_{k} \rightarrow 0, \quad \text { and } \quad s_{k}\left|u_{t t}\left(s_{k}, x\right)\right|_{2}^{2} \rightarrow 0, \quad \text { as } \quad k \rightarrow+\infty .
$$

Taking $\tau=s_{k}$ and letting $k \rightarrow+\infty$ in (3.74), due to the Gronwall's inequality, one has

$$
\begin{gathered}
t\left|u_{t t}(t)\right|_{2}^{2}+\int_{0}^{t} s\left|\sqrt{h} \nabla u_{t t}\right|_{2}^{2} \mathrm{~d} s \leq C c_{4}^{4} \exp \left(c_{5}^{3} t\right) \leq C c_{4}^{4} \\
\int_{0}^{t} s\left|\nabla u_{t t}\right|_{2}^{2} \mathrm{~d} s \leq C c_{4}^{5} .
\end{gathered}
$$

According to (3.68), (3.70) and (3.75), it holds that

$$
t^{\frac{1}{2}}\left|\nabla^{2} u_{t}(t)\right|_{2} \leq C c_{0} c_{4}^{2}, \quad t^{\frac{1}{2}}\left|\nabla^{4} u(t)\right|_{2} \leq C c_{0}^{2} c_{4}^{2} .
$$


Then it follows from Lemma 2.5 and (3.67) that for $0 \leq t \leq T_{5}$,

$$
\begin{aligned}
\left|\sqrt{h^{2}+\epsilon^{2}} u_{t}(t)\right|_{D^{3} \leq} \leq & C\left|u_{t t}+(v \cdot \nabla v)_{t}+\nabla \phi_{t}-(\psi \cdot Q(v))_{t}+\frac{a h}{\sqrt{h^{2}+\epsilon^{2}}} h_{t} L u\right|_{D^{1}} \\
& +C\left|G\left(\nabla \sqrt{h^{2}+\epsilon^{2}}, u_{t}\right)\right|_{D^{1}} \\
\leq & C\left(\left|\nabla u_{t t}\right|_{2}+c_{4}^{10}+c_{4}\left(\left|v_{t}\right|_{D^{2}}+\left|u_{t}\right|_{D^{2}}\right)\right), \\
\left|\sqrt{h^{2}+\epsilon^{2}} \nabla^{3} u_{t}(t)\right|_{2} \leq & C\left(\left|\sqrt{h^{2}+\epsilon^{2}} u_{t}\right|_{D^{3}}+\left|u_{t}\right|_{\infty}\left|\nabla^{2} \psi\right|_{2}+\left|\nabla u_{t}\right|_{6}|\nabla \psi|_{3}\right. \\
& \left.+\left|\nabla^{2} u_{t}\right|_{2}|\psi|_{\infty}+\left.\left|\nabla u_{t}\right|_{2}\left|\psi \psi \|_{D^{1} \cap D^{2}}^{2}\right| \varphi\right|_{\infty}+\left|u_{t}\right|_{2}|\psi|_{\infty}^{3}|\varphi|_{2}^{2}\right),
\end{aligned}
$$

which, along with (3.61) and (3.75)-(3.77), implies that

$$
\int_{0}^{T_{5}} t\left(\left|\sqrt{h^{2}+\epsilon^{2}} u_{t}\right|_{D^{3}}^{2}+\left|h \nabla^{3} u_{t}\right|_{2}^{2}+\left|\nabla^{3} u_{t}\right|_{2}^{2}\right) \mathrm{d} t \leq C c_{4}^{7}
$$

Then by Lemmas 3.2 3.7, for $0 \leq t \leq T_{5}=\min \left(T^{*},\left(1+C c_{5}\right)^{-20}\right)$, one has

$$
\begin{aligned}
& \left(\left\|\phi-\phi^{\infty}\right\|_{3}^{2}+\left\|\phi_{t}\right\|_{2}^{2}+\left|\phi_{t t}\right|_{2}^{2}\right)(t)+\int_{0}^{t}\left\|\phi_{t t}\right\|_{1}^{2} \mathrm{~d} s \leq C c_{4}^{6}, \\
& \|\psi(t)\|_{D^{1} \cap D^{2}}^{2} \leq C c_{0}^{2}, \quad\left|\psi_{t}(t)\right|_{2} \leq C c_{3}^{2}, \\
& \left|h_{t}(t)\right|_{\infty}^{2} \leq C c_{3}^{3} c_{4}, \quad h(t, x)>\frac{1}{2 c_{0}}, \quad\left|\psi_{t}(t)\right|_{D^{1}}^{2}+\int_{0}^{t}\left(\left|\psi_{t t}\right|_{2}^{2}+\left|h_{t t}\right|_{6}^{2}\right) \mathrm{d} s \leq C c_{4}^{4}, \\
& \frac{2}{3} \eta^{-2 e}<\varphi,\left(\|\varphi\|_{L^{\infty} \cap D^{1,6} \cap D^{2,3} \cap D^{3}}^{2}+\|f\|_{L^{\infty} \cap L^{6} \cap D^{1,3} \cap D^{2}}^{2}\right)(t) \leq C c_{0}^{4}, \\
& \left(\left\|\varphi_{t}\right\|_{L^{6} \cap D^{1,3} \cap D^{2}}^{2}+\left\|f_{t}\right\|_{L^{3} \cap D^{1}}^{2}\right)(t) \leq C c_{4}^{10}, \\
& |\sqrt{h} \nabla u|_{2}^{2}+\|u(t)\|_{1}^{2}+\int_{0}^{t}\left(\|\nabla u\|_{1}^{2}+\left|u_{t}\right|_{2}^{2}\right) \mathrm{d} s \leq C c_{0}^{4}, \\
& \left(|u|_{D^{2}}^{2}+\left|h \nabla^{2} u\right|_{2}^{2}+\left|u_{t}\right|_{2}^{2}\right)(t)+\int_{0}^{t}\left(|u|_{D^{3}}^{2}+\left|h \nabla^{2} u\right|_{D^{1}}^{2}+\left|u_{t}\right|_{D^{1}}^{2}\right) \mathrm{d} s \leq C c_{2}^{9} c_{3}, \\
& \left(\left|u_{t}\right|_{D^{1}}^{2}+\left|\sqrt{h} \nabla u_{t}\right|_{2}^{2}+|u|_{D^{3}}^{2}+\left|h \nabla^{2} u\right|_{D^{1}}^{2}\right)(t)+\int_{0}^{t}\left|u_{t}\right|_{D^{2}}^{2} \mathrm{~d} s \leq C c_{3}^{16}, \\
& \int_{0}^{t}\left(\left|u_{t t}\right|_{2}^{2}+|u|_{D^{4}}^{2}+|h \nabla u|_{D^{1}}^{2}+\left|h \nabla^{2} u\right|_{D^{2}}^{2}+\left|\left(h \nabla^{2} u\right)_{t}\right|_{2}^{2}\right) \mathrm{d} s \leq C c_{0}^{11}, \\
& t\left(\left|u_{t}\right|_{D^{2}}^{2}+\left|u_{t t}\right|_{2}^{2}+|u|_{D^{4}}^{2}\right)(t)+\int_{0}^{t}\left(s\left|u_{t t}\right|_{D^{1}}^{2}+s\left|u_{t}\right|_{D^{3}}^{2}\right) \mathrm{d} s \leq C c_{4}^{8} .
\end{aligned}
$$

Therefore, defining the time

$$
T^{*}=\min \left(T,\left(1+C^{\frac{357}{2}} c_{0}^{576}\right)^{-20}\right)
$$

and constants

$$
c_{1}=C^{\frac{1}{2}} c_{0}, \quad c_{2}=C^{\frac{1}{2}} c_{0}^{2}, \quad c_{3}=C^{\frac{11}{2}} c_{0}^{18}, \quad c_{4}=C^{\frac{89}{2}} c_{0}^{144}, \quad c_{5}=C^{\frac{357}{2}} c_{0}^{576},
$$


one can obtain

$$
\begin{aligned}
& \left(\left\|\phi-\phi^{\infty}\right\|_{3}^{2}+\left\|\phi_{t}\right\|_{2}^{2}+\left|\phi_{t t}\right|_{2}^{2}\right)(t)+\int_{0}^{t}\left\|\phi_{t t}\right\|_{1}^{2} \mathrm{~d} s \leq c_{5}^{2}, \\
& \|\psi(t)\|_{D^{1} \cap D^{2}}^{2} \leq c_{1}^{2}, \quad\left|h_{t}\right|_{\infty}^{2}+\left|\psi_{t}(t)\right|_{2} \leq c_{4}^{2}, \\
& \left|\psi_{t}(t)\right|_{D^{1}}^{2}+\int_{0}^{t}\left(\left|\psi_{t t}\right|_{2}^{2}+\left|h_{t t}\right|_{2}^{2}\right) \mathrm{d} s \leq c_{5}^{2}, \\
& h>\frac{1}{2 c_{0}}, \quad \frac{2}{3} \eta^{-2 e}<\varphi, \quad\left(\|\varphi\|_{L^{\infty} \cap D^{1,6} \cap D^{2,3} \cap D^{3}}^{2}+\|f\|_{L^{\infty} \cap L^{6} \cap D^{1,3} \cap D^{2}}^{2}\right)(t) \leq c_{2}^{2}, \\
& \left(\left\|\varphi_{t}\right\|_{L^{6} \cap D^{1,3} \cap D^{2}}^{2}+\left\|f_{t}\right\|_{L^{3} \cap D^{1}}^{2}\right)(t) \leq c_{5}^{3}, \\
& |\sqrt{h} \nabla u|_{2}^{2}+\|u(t)\|_{1}^{2}+\int_{0}^{t}\left(\|\nabla u\|_{1}^{2}+\left|u_{t}\right|_{2}^{2}\right) \mathrm{d} s \leq c_{2}^{2}, \\
& \left(|u|_{D^{2}}^{2}+\left|h \nabla^{2} u\right|_{2}^{2}+\left|u_{t}\right|_{2}^{2}\right)(t)+\int_{0}^{t}\left(|u|_{D^{3}}^{2}+\left|h \nabla^{2} u\right|_{D^{1}}^{2}+\left|u_{t}\right|_{D^{1}}^{2}\right) \mathrm{d} s \leq c_{3}^{2}, \\
& \left(\left|\sqrt{h} \nabla u_{t}\right|_{2}^{2}+\left|u_{t}\right|_{D^{1}}^{2}+|u|_{D^{3}}^{2}+|h \nabla u|_{D^{1}}^{2}+\left|h \nabla^{2} u\right|_{D^{1}}^{2}\right)(t)+\int_{0}^{t}\left|u_{t}\right|_{D^{2}}^{2} \mathrm{~d} s \leq c_{4}^{2}, \\
& \int_{0}^{t}\left(\left|u_{t t}\right|_{2}^{2}+|u|_{D^{4}}^{2}+\left|h \nabla^{2} u\right|_{D^{2}}^{2}+\left|\left(h \nabla^{2} u\right)_{t}\right|_{2}^{2}\right) \mathrm{d} s \leq c_{4}^{2}, \\
& t\left(\left|u_{t}\right|_{D^{2}}^{2}+\left|u_{t t}\right|_{2}^{2}+|u|_{D^{4}}^{2}\right)(t)+\int_{0}^{t}\left(s\left|u_{t t}\right|_{D^{1}}^{2}+s\left|u_{t}\right|_{D^{3}}^{2}\right) \mathrm{d} s \leq c_{5}^{2},
\end{aligned}
$$

for $0 \leq t \leq T^{*}$. In another word, given fixed $c_{0}$ and $T$, there are positive constants $T^{*}$, $c_{i}(i=1,2,3,4,5)$, depending only on $c_{0}$ and $T$, such that if (3.24) holds for $(g, v)$, then (3.80) holds for the classical solution to (3.15) on $\left[0, T^{*}\right] \times \mathbb{R}^{3}$.

3.4. Passing to the limit $\epsilon \rightarrow 0$. With the help of the $(\epsilon, \eta)$-independent estimates established in (3.80), we now establish the local existence result for the following linearized problem without artificial viscosity (i.e., $\epsilon=0$ ) under the assumption $\phi_{0} \geq \eta$,

$$
\left\{\begin{array}{l}
\phi_{t}+v \cdot \nabla \phi+(\gamma-1) \phi \operatorname{div} v=0, \\
u_{t}+v \cdot \nabla v+\nabla \phi+a h L u=\psi \cdot Q(v), \\
h_{t}+v \cdot \nabla h+(\delta-1) g \operatorname{div} v=0, \\
\left.(\phi, u, h)\right|_{t=0}=\left(\phi_{0}, u_{0}, h_{0}\right)=\left(\phi_{0}, u_{0},\left(\phi_{0}\right)^{2 e}\right), \quad x \in \mathbb{R}^{3}, \\
(\phi, u, h) \rightarrow\left(\phi^{\infty}, 0, h^{\infty}=\left(\phi^{\infty}\right)^{2 e}\right), \quad \text { as } \quad|x| \rightarrow+\infty, \quad t>0 .
\end{array}\right.
$$

Lemma 3.8. Let (1.11) hold. Assume that the initial data $\left(\phi_{0}, u_{0}, h_{0}=\left(\phi_{0}\right)^{2 e}\right)$ satisfies the hypothesis of Lemma 3.1, and there exists a positive constant $c_{0}$ independent of $\eta$ such that (3.19) holds. Then there exist a time $T^{*}>0$ independent of $\eta$, and a unique classical solution

$$
\left(\phi, u, h, \psi=\frac{a \delta}{\delta-1} \nabla h\right)
$$


in $\left[0, T^{*}\right] \times \mathbb{R}^{3}$ to (3.81) satisfying (3.18) with $T$ replaced by $T^{*}$. Moreover, $(\phi, u, h)$ satisfies the estimates in (3.80) independent of $\eta$.

Proof. We shall prove the existence, uniqueness and time continuity in two steps.

Step 1: Existence. First, it follows from Lemmas 3.1 3.7 that for every $\epsilon>0$ and $\eta>0$, there exist a time $T^{*}>0$ independent of $(\epsilon, \eta)$, and a unique strong solution $\left(\phi^{\epsilon, \eta}, u^{\epsilon, \eta}, h^{\epsilon, \eta}\right)(t, x)$ in $\left[0, T^{*}\right] \times \mathbb{R}^{3}$ to the linearized problem (3.15) satisfying the estimates in (3.80), which are independent of $(\epsilon, \eta)$.

Second, using the characteristic method and the standard energy estimates for transport equations, and (3.15) 3 , one gets easily that

$$
\left\|h^{\epsilon, \eta}(t)\right\|_{L^{\infty} \cap D^{1}}+\left|h_{t}^{\epsilon, \eta}(t)\right|_{2} \leq C\left(\eta, \alpha, \beta, \gamma, \delta, T, \phi_{0}, u_{0}\right), \quad \text { for } \quad 0 \leq t \leq T^{*} .
$$

Then, by virtue or the uniform estimates in (3.80) independent of $(\epsilon, \eta)$, estimates in (3.82) independent of $\epsilon$, and the compactness in Lemma 2.2 (see [26]), one gets that for any $R>0$, there exists a subsequence of solutions (still denoted by) $\left(\phi^{\epsilon, \eta}, u^{\epsilon, \eta}, h^{\epsilon, \eta}\right)$, which converges to a limit $\left(\phi^{\eta}, u^{\eta}, h^{\eta}\right)$ in the following strong sense:

$$
\left(\phi^{\epsilon, \eta}, u^{\epsilon, \eta}, h^{\epsilon, \eta}\right) \rightarrow\left(\phi^{\eta}, u^{\eta}, h^{\eta}\right) \quad \text { in } C\left(\left[0, T^{*}\right] ; H^{2}\left(B_{R}\right)\right), \quad \text { as } \quad \epsilon \rightarrow 0 .
$$

Again, due to the uniform estimates in (3.80) independent of $\eta$ and the estimates in (3.82) independent of $\epsilon$, there exists a subsequence (of subsequence chosen above) of solutions (still denoted by) $\left(\phi^{\epsilon, \eta}, u^{\epsilon, \eta}, h^{\epsilon, \eta}\right)$, which converges to $\left(\phi^{\eta}, u^{\eta}, h^{\eta}\right)$ as $\epsilon \rightarrow 0$ in the following weak or weak* sense:

$$
\begin{aligned}
& \left(\phi^{\epsilon, \eta}-\phi^{\infty}, u^{\epsilon, \eta}\right) \rightarrow\left(\phi^{\eta}-\phi^{\infty}, u^{\eta}\right) \quad \text { weakly* in } L^{\infty}\left(\left[0, T^{*}\right] ; H^{3}\right), \\
& \left(\phi_{t}^{\epsilon, \eta}, \psi^{\epsilon, \eta}, h_{t}^{\epsilon, \eta}\right) \rightarrow\left(\phi_{t}^{\eta}, \psi^{\eta}, h_{t}^{\eta}\right) \quad \text { weakly* in } L^{\infty}\left(\left[0, T^{*}\right] ; H^{2}\right), \\
& u_{t}^{\epsilon, \eta} \rightarrow u_{t}^{\eta} \quad \text { weakly* in } L^{\infty}\left(\left[0, T^{*}\right] ; H^{1}\right) \text {, } \\
& \left(\phi_{t t}^{\epsilon, \eta}, \nabla^{3} \varphi^{\epsilon, \eta}, \nabla^{2} f^{\epsilon, \eta}\right) \rightarrow\left(\phi_{t t}^{\eta}, \nabla^{3} \varphi^{\eta}, \nabla^{2} f^{\eta}\right) \quad \text { weakly* in } L^{\infty}\left(\left[0, T^{*}\right] ; L^{2}\right), \\
& \left(\nabla^{2} \varphi_{t}^{\epsilon, \eta}, \nabla f_{t}^{\epsilon, \eta}\right) \rightarrow\left(\nabla^{2} \varphi_{t}^{\eta}, \nabla f_{t}^{\eta}\right) \quad \text { weakly* in } L^{\infty}\left(\left[0, T^{*}\right] ; L^{2}\right), \\
& t^{\frac{1}{2}}\left(\nabla^{2} u^{\epsilon, \eta}, u_{t t}^{\epsilon, \eta}, \nabla^{4} u^{\epsilon, \eta}\right) \rightarrow t^{\frac{1}{2}}\left(\nabla^{2} u^{\eta}, u_{t t}^{\eta}, \nabla^{4} u^{\eta}\right) \quad \text { weakly* in } L^{\infty}\left(\left[0, T^{*}\right] ; L^{2}\right), \\
& \left(\nabla^{2} \varphi^{\epsilon, \eta}, \nabla \varphi_{t}^{\epsilon, \eta}, \nabla f^{\epsilon, \eta}, f_{t}^{\epsilon, \eta}\right) \rightarrow\left(\nabla^{2} \varphi^{\eta}, \nabla \varphi_{t}^{\eta}, \nabla f^{\eta}, f_{t}^{\eta}\right) \quad \text { weakly* in } L^{\infty}\left(\left[0, T^{*}\right] ; L^{3}\right) \text {, } \\
& \left(\nabla \varphi^{\epsilon, \eta}, \varphi_{t}^{\epsilon, \eta}, f^{\epsilon, \eta}\right) \rightarrow\left(\nabla \varphi^{\eta}, \varphi_{t}^{\eta}, f^{\eta}\right) \quad \text { weakly* in } L^{\infty}\left(\left[0, T^{*}\right] ; L^{6}\right), \\
& \left(h^{\epsilon, \eta}, h_{t}^{\epsilon, \eta}, \varphi^{\epsilon, \eta}, f^{\epsilon, \eta}\right) \rightarrow\left(h^{\eta}, h_{t}^{\eta}, \varphi^{\eta}, f^{\eta}\right) \quad \text { weakly* in } L^{\infty}\left(\left[0, T^{*}\right] ; L^{\infty}\right) \text {, } \\
& \nabla u^{\epsilon, \eta} \rightarrow \nabla u^{\eta} \quad \text { weakly in } \quad L^{2}\left(\left[0, T^{*}\right] ; H^{3}\right) \text {, } \\
& u_{t}^{\epsilon, \eta} \rightarrow u_{t}^{\eta} \quad \text { weakly } \quad \text { in } \quad L^{2}\left(\left[0, T^{*}\right] ; H^{2}\right), \\
& \phi_{t t}^{\epsilon, \eta} \rightarrow \phi_{t t}^{\eta} \quad \text { weakly } \quad \text { in } \quad L^{2}\left(\left[0, T^{*}\right] ; H^{1}\right), \\
& \left(\psi_{t t}^{\epsilon, \eta}, h_{t t}^{\epsilon, \eta}, u_{t t}^{\epsilon, \eta}\right) \rightarrow\left(\psi_{t t}^{\eta}, h_{t t}^{\eta}, u_{t t}^{\eta}\right) \quad \text { weakly in } L^{2}\left(\left[0, T^{*}\right] ; L^{2}\right), \\
& \left.t^{\frac{1}{2}}\left(\nabla u_{t t}^{\epsilon, \eta}, \nabla^{3} u_{t}^{\epsilon, \eta}\right) \rightarrow t^{\frac{1}{2}}\left(\nabla u_{t t}^{\eta}, \nabla^{3} u^{\eta}\right)_{t}\right) \quad \text { weakly in } L^{2}\left(\left[0, T^{*}\right] ; L^{2}\right) \text {, }
\end{aligned}
$$

which, along with the lower semi-continuity of weak or weak* convergence, implies that $\left(\phi^{\eta}, u^{\eta}, h^{\eta}\right)$ satisfies also the corresponding estimates in (3.80) and (3.82) except those weighted estimates on $u^{\eta}$. 
Collecting the uniform estimates on $\left(\phi^{\eta}, u^{\eta}, h^{\eta}\right)$ obtained above, together with the strong convergence in (3.83) and the weak or weak* convergence in (3.84), one obtains that

$$
\begin{aligned}
& \sqrt{h^{\epsilon, \eta}}\left(\nabla u^{\epsilon, \eta}, \nabla u_{t}^{\epsilon, \eta}\right) \rightarrow \sqrt{h^{\eta}}\left(\nabla u^{\eta}, \nabla u_{t}^{\eta}\right) \quad \text { weakly* in } L^{\infty}\left(\left[0, T^{*}\right] ; L^{2}\right), \\
& h^{\epsilon, \eta} \nabla^{2} u^{\epsilon, \eta} \rightarrow h^{\eta} \nabla^{2} u^{\eta} \quad \text { weakly* in } L^{\infty}\left(\left[0, T^{*}\right] ; H^{1}\right), \\
& \left(h^{\epsilon, \eta} \nabla^{2} u^{\epsilon, \eta}\right)_{t} \rightarrow\left(h^{\eta} \nabla^{2} u^{\eta}\right)_{t} \quad \text { weakly } \quad \text { in } \quad L^{2}\left(\left[0, T^{*}\right] ; L^{2}\right) \text {, } \\
& h^{\epsilon, \eta} \nabla^{2} u^{\epsilon, \eta} \rightarrow h^{\eta} \nabla^{2} u^{\eta} \quad \text { weakly } \quad \text { in } \quad L^{2}\left(\left[0, T^{*}\right] ; D^{1} \cap D^{2}\right),
\end{aligned}
$$

which, along with the lower semi-continuity of weak or weak* convergence again, implies that $\left(\phi^{\eta}, u^{\eta}, h^{\eta}\right)$ satisfies also the uniform weighted estimates on $u^{\eta}$.

Now we are going to show that $\left(\phi^{\eta}, u^{\eta}, h^{\eta}\right)$ is a weak solution in the sense of distribution to (3.81). First, multiplying (3.81) 2 by test function $w(t, x)=\left(w^{1}, w^{2}, w^{3}\right) \in C_{c}^{\infty}\left(\left[0, T^{*}\right) \times\right.$ $\left.\mathbb{R}^{3}\right)$ on both sides, and integrating over $[0, t) \times \mathbb{R}^{3}$ for $t \in\left(0, T^{*}\right]$, one has

$$
\begin{aligned}
& \int_{0}^{t} \int\left(u^{\epsilon, \eta} \cdot w_{t}-(v \cdot \nabla) v \cdot w+\phi^{\epsilon, \eta} \operatorname{div} w\right) \mathrm{d} x \mathrm{~d} s \\
= & -\int u_{0} \cdot w(0, x)+\int_{0}^{t} \int\left(\sqrt{\left(h^{\epsilon, \eta}\right)^{2}+\epsilon^{2}} L u^{\epsilon, \eta} \cdot w-\psi^{\epsilon, \eta} \cdot Q(v) \cdot w\right) \mathrm{d} x \mathrm{~d} s .
\end{aligned}
$$

It follows from the uniform estimates obtained above, the strong convergence in (3.83), and the weak convergences in (3.84)-(3.85) that and letting $\epsilon \rightarrow 0$ in (3.86) yields

$$
\begin{aligned}
& \int_{0}^{t} \int\left(u^{\eta} \cdot w_{t}-(v \cdot \nabla) v \cdot w+\phi^{\eta} \operatorname{div} w\right) \mathrm{d} x \mathrm{~d} s \\
= & -\int u_{0} \cdot w(0, x)+\int_{0}^{t} \int\left(h^{\eta} L u^{\eta} \cdot w-\psi^{\eta} \cdot Q(v) \cdot w\right) \mathrm{d} x \mathrm{~d} s .
\end{aligned}
$$

Second, one can use the similar argument to show that $\left(\phi^{\eta}, h^{\eta}\right)$ satisfies also the equations in (3.81) 1 and (3.81) 3 and the initial data in the sense of distribution. So it is clear that $\left(\phi^{\eta}, u^{\eta}, h^{\eta}\right)$ is a weak solution in the sense of distribution to the linearized problem (3.81), satisfying the following regularities

$$
\begin{aligned}
& \phi^{\eta}-\phi^{\infty} \in L^{\infty}\left(\left[0, T^{*}\right] ; H^{3}\right), \quad h^{\eta} \in L^{\infty}\left(\left[0, T^{*}\right] \times \mathbb{R}^{3}\right), \quad \nabla h^{\eta} \in L^{\infty}\left(\left[0, T^{*}\right] ; H^{2}\right), \\
& h_{t}^{\eta} \in L^{\infty}\left(\left[0, T^{*}\right] ; H^{2}\right), \quad u^{\eta} \in L^{\infty}\left([0, T] ; H^{3}\right) \cap L^{2}\left(\left[0, T^{*}\right] ; H^{4}\right), \\
& u_{t}^{\eta} \in L^{\infty}\left(\left[0, T^{*}\right] ; H^{1}\right) \cap L^{2}\left(\left[0, T^{*}\right] ; D^{2}\right), \quad u_{t t}^{\eta} \in L^{2}\left(\left[0, T^{*}\right] ; L^{2}\right), \\
& t^{\frac{1}{2}} u^{\eta} \in L^{\infty}\left(\left[0, T^{*}\right] ; D^{4}\right), \quad t^{\frac{1}{2}} u_{t}^{\eta} \in L^{\infty}\left(\left[0, T^{*}\right] ; D^{2}\right) \cap L^{2}\left(\left[0, T^{*}\right] ; D^{3}\right), \\
& t^{\frac{1}{2}} u_{t t}^{\eta} \in L^{\infty}\left(\left[0, T^{*}\right] ; L^{2}\right) \cap L^{2}\left(\left[0, T^{*}\right] ; D^{1}\right) .
\end{aligned}
$$

Therefore, this weak solution $\left(\phi^{\eta}, u^{\eta}, h^{\eta}\right)$ of (3.81) is actually a strong one.

Step 2: Uniqueness and Time continuity. Due to the estimate $h^{\eta}>\frac{1}{2 c_{0}}$, the uniqueness and the time continuity of the strong solution obtained above can be proved via the completely same arguments as in Lemma 3.1, so details are omitted. 
3.5. Construction of the nonlinear approximation solutions away from vacuum. In this subsection, based on the assumption that $\phi_{0}>\eta$, we will prove the local-in-time well-posedness of the classical solution to the following Cauchy problem

$$
\left\{\begin{array}{l}
\phi_{t}+u \cdot \nabla \phi+(\gamma-1) \phi \operatorname{div} u=0, \\
u_{t}+u \cdot \nabla u+\nabla \phi+a \phi^{2 e} L u=\psi \cdot Q(u), \\
\psi_{t}+\nabla(u \cdot \psi)+(\delta-1) \psi \operatorname{div} u+\delta a \phi^{2 e} \nabla \operatorname{div} u=0, \\
\left.(\phi, u, \psi)\right|_{t=0}=\left(\phi_{0}, u_{0}, \psi_{0}\right)=\left(\phi_{0}, u_{0}, \frac{a \delta}{\delta-1} \nabla\left(\phi_{0}\right)^{2 e}\right), \quad x \in \mathbb{R}^{3}, \\
(\phi, u, \psi) \rightarrow\left(\phi^{\infty}, 0,0\right), \quad \text { as } \quad|x| \rightarrow+\infty, \quad t \geq 0,
\end{array}\right.
$$

whose life span is independent of $\eta$.

Theorem 3.2. Let (1.11) hold and $\phi^{\infty}$ be a postive constant. Assume that the initial data $\left(\phi_{0}, u_{0}, h_{0}=\left(\phi_{0}\right)^{2 e}, \psi_{0}=\frac{a \delta}{\delta-1} \nabla\left(\phi_{0}\right)^{2 e}\right)$ satisfies the hypothesis of Lemma [3.1, and there exists a positive constant $c_{0}$ independent of $\eta$ such that (3.19) holds. Then there exist a time $T_{*}>0$ and a unique classical solution

$$
\left(\phi, u, h=\phi^{2 e}, \psi=\frac{a \delta}{\delta-1} \nabla h\right)
$$

in $\left[0, T_{*}\right] \times \mathbb{R}^{3}$ to the Cauchy problem (3.89) satisfying (3.18) and

$$
\phi^{-2 e} \in L^{\infty}\left(\left[0, T_{*}\right] ; L^{\infty} \cap D^{1,6} \cap D^{2,3} \cap D^{3}\right), \quad \nabla \phi / \phi \in L^{\infty}\left(\left[0, T_{*}\right] ; L^{\infty} \cap L^{6} \cap D^{1,3} \cap D^{2}\right),
$$

where $T_{*}$ is independent of $\eta$. Moreover, if the initial data satisfies (3.19), then estimates (3.80) hold for $(\phi, u, h)$ with $T^{*}$ replaced by $T_{*}$, and are independent of $\eta$.

The proof is given by an iteration scheme based on the estimates for the linearized problem obtained in Sections 3.2-3.4. As in Section 3.3, we define constants $c_{i}(i=1, \ldots, 5)$.

Let $\left(\phi^{0}, u^{0}, h^{0}\right)$ be the solution to the following Cauchy problem

$$
\left\{\begin{array}{l}
X_{t}+u_{0} \cdot \nabla X=0 \quad \text { in } \quad(0,+\infty) \times \mathbb{R}^{3}, \\
Y_{t}-Z \triangle Y=0 \quad \text { in } \quad(0,+\infty) \times \mathbb{R}^{3}, \\
Z_{t}+u_{0} \cdot \nabla Z=0 \quad \text { in } \quad(0,+\infty) \times \mathbb{R}^{3}, \\
\left.(X, Y, Z)\right|_{t=0}=\left(\phi_{0}, u_{0}, h_{0}\right)=\left(\phi_{0}, u_{0}, \phi_{0}^{2 e}\right) \quad \text { in } \quad \mathbb{R}^{3}, \\
(X, Y, Z) \rightarrow\left(\phi^{\infty}, 0, h^{\infty}=\left(\phi^{\infty}\right)^{2 e}\right) \quad \text { as } \quad|x| \rightarrow+\infty, \quad t>0 .
\end{array}\right.
$$


Choose a time $T^{* *} \in\left(0, T^{*}\right]$ small enough such that

$$
\begin{array}{r}
\sup _{0 \leq t \leq T^{* *}}\left\|\left.\nabla h^{0}(t)\right|_{D^{1} \cap D^{2}} ^{2} \leq c_{1}^{2}, \sup _{0 \leq t \leq T^{* *}}\right\| u^{0}(t) \|_{1}^{2}+\int_{0}^{T^{* *}}\left(\left|u^{0}\right|_{D^{2}}^{2}+\left|u_{t}^{0}\right|_{2}^{2}\right) \mathrm{d} t \leq c_{2}^{2}, \\
\sup _{0 \leq t \leq T^{* *}}\left(\left|u^{0}\right|_{D^{2}}^{2}+\left|u_{t}^{0}\right|_{2}^{2}+\left|h^{0} \nabla^{2} u^{0}\right|_{2}^{2}\right)(t)+\int_{0}^{T^{* *}}\left(\left|u^{0}\right|_{D^{3}}^{2}+\left|u_{t}^{0}\right|_{D^{1}}^{2}\right) \mathrm{d} t \leq c_{3}^{2}, \\
\sup _{0 \leq t \leq T^{* *}}\left(\left|u^{0}\right|_{D^{3}}^{2}+\left|u_{t}^{0}\right|_{D^{1}}^{2}+\left|h_{t}^{0}\right|_{D^{1}}^{2}\right)(t)+\int_{0}^{T^{* *}}\left(\left|u^{0}\right|_{D^{4}}^{2}+\left|u_{t}^{0}\right|_{D^{2}}^{2}+\left|u_{t t}^{0}\right|_{2}^{2}\right) \mathrm{d} t \leq c_{4}^{2}, \\
\sup _{0 \leq t \leq T^{* *}}\left(\left|h^{0} \nabla^{2} u^{0}\right|_{D^{1}}^{2}+\left|h_{t}^{0}\right|_{\infty}^{2}\right)(t)+\int_{0}^{T^{* *}}\left(\left|\left(h^{0} \nabla^{2} u^{0}\right)_{t}\right|_{2}^{2}+\left|h^{0} \nabla^{2} u^{0}\right|_{D^{2}}^{2}\right) \mathrm{d} t \leq c_{4}^{2}, \\
\operatorname{ess}_{0 \leq t \leq T^{* *}} t\left(\left|u_{t}^{0}\right|_{D^{2}}^{2}+\left|u^{0}\right|_{D^{4}}^{2}+\left|u_{t t}^{0}\right|_{2}^{2}\right)(t)+\int_{0}^{T^{* *}} t\left(\left|u_{t t}^{0}\right|_{D^{1}}^{2}+\left|u_{t}^{0}\right|_{D^{3}}^{2}\right) \mathrm{d} t \leq c_{5}^{2} .
\end{array}
$$

Proof. The existence, uniqueness and time continuity can be proved as follows.

Step 1: Existence. Let the beginning step of our iteration be $(v, g)=\left(u^{0}, h^{0}\right)$. Thus one can get a classical solution $\left(\phi^{1}, u^{1}, h^{1}\right)$ of problem (3.81). Inductively, one constructs approximate sequences $\left(\phi^{k+1}, u^{k+1}, h^{k+1}\right)$ as follows: given $\left(u^{k}, h^{k}\right)$ for $k \geq 1$, define $\left(\phi^{k+1}, u^{k+1}, h^{k+1}\right)$ by solving the following problem:

$$
\left\{\begin{array}{l}
\phi_{t}^{k+1}+u^{k} \cdot \nabla \phi^{k+1}+(\gamma-1) \phi^{k+1} \operatorname{div} u^{k}=0, \\
u_{t}^{k+1}+u^{k} \cdot \nabla u^{k}+\nabla \phi^{k+1}+a h^{k+1} L u^{k+1}=\psi^{k+1} \cdot Q\left(u^{k}\right), \\
h_{t}^{k+1}+u^{k} \cdot \nabla h^{k+1}+(\delta-1) h^{k} \operatorname{div} u^{k}=0, \\
\left.\left(\phi^{k+1}, u^{k+1}, h^{k+1}\right)\right|_{t=0}=\left(\phi_{0}, u_{0}, \phi_{0}^{2 e}\right), \\
\left(\phi^{k+1}, u^{k+1}, h^{k+1}\right) \rightarrow\left(\phi^{\infty}, 0, h^{\infty}=\left(\phi^{\infty}\right)^{2 e}\right), \quad \text { as } \quad|x| \rightarrow+\infty, \quad t \geq 0 .
\end{array}\right.
$$

This problem can be solved from (3.81) by replacing $(v, g)$ with $\left(u^{k}, h^{k}\right)$, and $\left(\phi^{k}, u^{k}, h^{k}\right)$ $(k=1,2, \ldots)$ satisfy the uniform estimates (3.80).

Denote

$$
\psi^{k+1}=\frac{a \delta}{\delta-1} \nabla h^{k+1}, \quad f^{k+1}=\left(h^{k+1}\right)^{-1} \psi^{k+1}=\frac{a \delta}{\delta-1} \nabla h^{k+1} / h^{k+1}, \quad \varphi^{k+1}=\left(h^{k+1}\right)^{-1}
$$


Then problem (3.92) can be rewritten as

$$
\left\{\begin{array}{l}
\phi_{t}^{k+1}+u^{k} \cdot \nabla \phi^{k+1}+(\gamma-1) \phi^{k+1} \operatorname{div} u^{k}=0, \\
\varphi^{k+1}\left(u_{t}^{k+1}+u^{k} \cdot \nabla u^{k}+\nabla \phi^{k+1}\right)=-a L u^{k+1}+f^{k+1} \cdot Q\left(u^{k}\right), \\
f_{t}^{k+1}+\sum_{l=1}^{3} A_{l}\left(u^{k}\right) \partial_{l} f^{k+1}+B^{*}\left(u^{k}\right) f^{k+1}+a \delta\left(\varphi^{k}\right)^{-1} \varphi^{k+1} \nabla \operatorname{div} u^{k} \\
=-a \delta \varphi^{k+1} \nabla h^{k} \operatorname{div} u^{k}+(\delta-1)\left(\varphi^{k}\right)^{-1} \varphi^{k+1} f^{k+1} \operatorname{div} u^{k}, \\
\\
\varphi_{t}^{k+1}+u^{k} \cdot \nabla \varphi^{k+1}-(\delta-1)\left(\varphi^{k}\right)^{-1}\left(\varphi^{k+1}\right)^{2} \operatorname{div} u^{k}=0, \\
\\
\left.\left(\phi^{k+1}, u^{k+1}, f^{k+1}, \varphi^{k+1}\right)\right|_{t=0}=\left(\phi_{0}, u_{0}, \phi_{0}^{-2 e} \psi_{0}, \phi_{0}^{-2 e}\right), \\
\left(\phi^{k+1}, u^{k+1}, f^{k+1}, \varphi^{k+1}\right) \rightarrow\left(\phi^{\infty}, 0,0,\left(\phi^{\infty}\right)^{-2 e}\right), \text { as }|x| \rightarrow+\infty, t \geq 0
\end{array}\right.
$$

Step 1.1: Strong convergence of $\left(\phi^{k}, u^{k}, f^{k}, \varphi^{k}\right)$. Now we are going to prove that the whole sequence $\left(\phi^{k}, u^{k}, f^{k}, \varphi^{k}\right)$ converges to a limit $(\phi, u, f, \varphi)$ in some strong sense. Set

$$
\bar{\phi}^{k+1}=\phi^{k+1}-\phi^{k}, \bar{u}^{k+1}=u^{k+1}-u^{k}, \bar{f}^{k+1}=f^{k+1}-f^{k}, \bar{\varphi}^{k+1}=\varphi^{k+1}-\varphi^{k} .
$$

Notice that

$$
\begin{aligned}
\left(\varphi^{k}\right)^{-1} \varphi^{k+1} \nabla \operatorname{div} u^{k} & =\bar{\varphi}^{k+1} h^{k} \nabla \operatorname{div} u^{k}+\nabla \operatorname{div} u^{k}, \\
\varphi^{k+1} \nabla h^{k} \operatorname{div} u^{k} & =\frac{\delta-1}{a \delta} f^{k} \operatorname{div} u^{k}\left(1+\bar{\varphi}^{k+1} h^{k}\right), \\
\left(\varphi^{k}\right)^{-1} \varphi^{k+1} f^{k+1} \operatorname{div} u^{k} & =f^{k+1} \operatorname{div} u^{k}\left(1+\bar{\varphi}^{k+1} h^{k}\right), \\
\left(\varphi^{k}\right)^{-1}\left(\varphi^{k+1}\right)^{2} \operatorname{div} u^{k} & =\bar{\varphi}^{k+1} \varphi^{k+1} h^{k} \operatorname{div} u^{k}+\varphi^{k} \operatorname{div} u^{k}+\bar{\varphi}^{k+1} \operatorname{div} u^{k} .
\end{aligned}
$$

Then it follows from (3.93) that

$$
\left\{\begin{array}{c}
\bar{\phi}_{t}^{k+1}+u^{k} \cdot \nabla \bar{\phi}^{k+1}+\bar{u}^{k} \cdot \nabla \phi^{k}+(\gamma-1)\left(\bar{\phi}^{k+1} \operatorname{div} u^{k}+\phi^{k} \operatorname{div} \bar{u}^{k}\right)=0, \\
\varphi^{k+1}\left(\bar{u}_{t}^{k+1}+u^{k} \cdot \nabla \bar{u}^{k}+\bar{u}^{k} \cdot \nabla u^{k-1}\right)+\varphi^{k+1} \nabla \bar{\phi}^{k+1}+\bar{\varphi}^{k+1} \nabla \phi^{k}+a L \bar{u}^{k+1} \\
=-\bar{\varphi}^{k+1}\left(u_{t}^{k}+u^{k-1} \cdot \nabla u^{k-1}\right)+f^{k+1} \cdot Q\left(\bar{u}^{k}\right)+\bar{f}^{k+1} \cdot Q\left(u^{k-1}\right), \\
\bar{f}_{t}^{k+1}+\sum_{l=1}^{3} A_{l}\left(u^{k}\right) \partial_{l} \bar{f}^{k+1}+B^{*}\left(u^{k}\right) \bar{f}^{k+1}+a \delta \nabla \operatorname{div} \bar{u}^{k}=\Upsilon_{1}^{k}+\Upsilon_{2}^{k}+\Upsilon_{3}^{k}, \\
\bar{\varphi}_{t}^{k+1}+u^{k} \cdot \nabla \bar{\varphi}^{k+1}+\bar{u}^{k} \cdot \nabla \varphi^{k}+(1-\delta)\left(\bar{\varphi}^{k} \operatorname{div} u^{k}+\varphi^{k-1} \operatorname{div} \bar{u}^{k}+\Upsilon_{4}^{k}\right)=0,
\end{array}\right.
$$


where $\Upsilon_{i}^{k}(i=1, \ldots, 4)$ are defined respectively as:

$$
\begin{aligned}
\Upsilon_{1}^{k}= & (1-\delta)\left(\bar{f}^{k} \operatorname{div} u^{k}+f^{k-1} \operatorname{div} \bar{u}^{k}+\bar{\varphi}^{k+1} f^{k} h^{k} \operatorname{div} u^{k}-\bar{\varphi}^{k} f^{k-1} h^{k-1} \operatorname{div} u^{k-1}\right), \\
\Upsilon_{2}^{k}= & -\sum_{l=1}^{3}\left(A_{l}\left(u^{k}\right)-A_{l}\left(u^{k-1}\right)\right) \partial_{l} f^{k}-\left(B^{*}\left(u^{k}\right)-B^{*}\left(u^{k-1}\right)\right) f^{k} \\
& -a \delta\left(\bar{\varphi}^{k+1} h^{k} \nabla \operatorname{div} u^{k}-\bar{\varphi}^{k} h^{k-1} \nabla \operatorname{div} u^{k-1}\right), \\
\Upsilon_{3}^{k}= & (\delta-1)\left(f^{k+1} \operatorname{div} u^{k} \bar{\varphi}^{k+1} h^{k}-f^{k} \operatorname{div} u^{k-1} \bar{\varphi}^{k} h^{k-1}+\bar{f}^{k+1} \operatorname{div} u^{k}+f^{k} \operatorname{div} \bar{u}^{k}\right), \\
\Upsilon_{4}^{k}= & \bar{\varphi}^{k+1} \varphi^{k+1} h^{k} \operatorname{div} u^{k}-\bar{\varphi}^{k} \varphi^{k} h^{k-1} \operatorname{div} u^{k-1}+\bar{\varphi}^{k+1} \operatorname{div} u^{k}-\bar{\varphi}^{k} \operatorname{div} u^{k-1} .
\end{aligned}
$$

For $\bar{\phi}^{k+1}$, multiplying (3.94) 1 by $2 \bar{\phi}^{k+1}$ and integrating over $\mathbb{R}^{3}$ give

$$
\frac{d}{d t}\left|\bar{\phi}^{k+1}\right|_{2}^{2} \leq C\left(\left|\nabla u^{k}\right|_{\infty}\left|\bar{\phi}^{k+1}\right|_{2}+\left|\bar{u}^{k}\right|_{6}\left|\nabla \phi^{k}\right|_{3}+\left|\nabla \bar{u}^{k}\right|_{2}\left|\phi^{k}\right|_{\infty}\right)\left|\bar{\phi}^{k+1}\right|_{2} .
$$

Then, applying derivative $\partial_{x}^{\zeta}(|\zeta|=1)$ to $(\underline{3.94})_{1}$, multiplying by $2 \partial_{x}^{\zeta} \bar{\phi}^{k+1}$ and integrating over $\mathbb{R}^{3}$, one gets

$$
\begin{aligned}
\frac{d}{d t}\left|\partial_{x}^{\zeta} \bar{\phi}^{k+1}\right|_{2}^{2} \leq & C\left(\left|\nabla u^{k}\right|_{\infty}\left|\nabla \bar{\phi}^{k+1}\right|_{2}+\left|\nabla \phi^{k}\right|_{\infty}\left|\nabla \bar{u}^{k}\right|_{2}+\left|\bar{u}^{k}\right|_{6}\left|\nabla^{2} \phi^{k}\right|_{3}\right)\left|\nabla \bar{\phi}^{k+1}\right|_{2} \\
& +C\left(\left|\nabla^{2} u^{k}\right|_{3}\left|\bar{\phi}^{k+1}\right|_{6}+\left|\nabla \bar{u}^{k}\right|_{2}\left|\nabla \phi^{k}\right|_{\infty}+\left|\phi^{k}\right|_{\infty}\left|\nabla \operatorname{div} \bar{u}^{k}\right|_{2}\right)\left|\nabla \bar{\phi}^{k+1}\right|_{2},
\end{aligned}
$$

which, along with (3.95), implies that for $t \in\left[0, T^{* *}\right]$,

$$
\frac{d}{d t}\left\|\bar{\phi}^{k+1}(t)\right\|_{1}^{2} \leq C \sigma^{-1}\left\|\bar{\phi}^{k+1}(t)\right\|_{1}^{2}+\sigma\left\|\nabla \bar{u}^{k}(t)\right\|_{1}^{2},
$$

where $\sigma \in(0,1)$ is a constant to be determined.

For $\bar{f}^{k+1}$, multiplying (3.94) 3 by $2 \bar{f}^{k+1}$ and integrating over $\mathbb{R}^{3}$ yield

$$
\begin{aligned}
\frac{d}{d t}\left|\bar{f}^{k+1}\right|_{2}^{2} & \leq C\left|\nabla u^{k}\right|_{\infty}\left|\bar{f}^{k+1}\right|_{2}^{2}+C\left(\sum_{i=1}^{3}\left|\Upsilon_{i}^{k}\right|_{2}+\left|\nabla^{2} \bar{u}^{k}\right|_{2}\right)\left|\bar{f}^{k+1}\right|_{2} \\
& \leq C \sigma^{-1}\left|\bar{f}^{k+1}\right|_{2}^{2}+C\left\|\bar{\varphi}^{k+1}\right\|_{1}^{2}+\sigma\left(\left\|\nabla \bar{u}^{k}\right\|_{1}^{2}+\left\|\bar{\varphi}^{k}\right\|_{1}^{2}+\left|\bar{f}^{k}\right|_{2}^{2}\right),
\end{aligned}
$$

where one has used the fact that

$$
\begin{aligned}
\left|\Upsilon_{1}^{k}\right|_{2} \leq & C\left(\left|\bar{f}^{k}\right|_{2}\left|\nabla u^{k}\right|_{\infty}+\left|\nabla \bar{u}^{k}\right|_{2}\left|f^{k-1}\right|_{\infty}+\left|f^{k}\right|_{\infty}\left|\bar{\varphi}^{k+1}\right|_{2}\left|h^{k} \nabla u^{k}\right|_{\infty}\right) \\
& +C\left|f^{k-1}\right|_{\infty}\left|h^{k-1} \nabla u^{k-1}\right|_{\infty}\left|\bar{\varphi}^{k}\right|_{2}, \\
\left|\Upsilon_{2}^{k}\right|_{2} \leq & C\left(\left|\nabla f^{k}\right|_{3}\left|\bar{u}^{k}\right|_{6}+\left|f^{k}\right|_{\infty}\left|\nabla \bar{u}^{k}\right|_{2}\right) \\
& +C\left(\left|\bar{\varphi}^{k+1}\right|_{6}\left|h^{k} \nabla \operatorname{div} u^{k}\right|_{3}+\left|\bar{\varphi}^{k}\right|_{6}\left|h^{k-1} \nabla \operatorname{div} u^{k-1}\right|_{3}\right), \\
\left|\Upsilon_{3}^{k}\right|_{2} \leq & C\left(\left|f^{k+1}\right|_{\infty}\left|\bar{\varphi}^{k+1}\right|_{2}\left|h^{k} \operatorname{div} u^{k}\right|_{\infty}+\left|f^{k}\right|_{\infty}\left|h^{k-1} \operatorname{div} u^{k-1}\right|_{\infty}\left|\bar{\varphi}^{k}\right|_{2}\right) \\
& +\left(\left|\nabla u^{k}\right|_{\infty}\left|\bar{f}^{k+1}\right|_{2}+\left|f^{k}\right|_{\infty}\left|\nabla \bar{u}^{k}\right|_{2}\right) .
\end{aligned}
$$

In the rest of the proof of this subsection, set 


$$
\begin{aligned}
R^{k}(t) & =\left(\left|\nabla u^{k}\right|_{\infty}+\left|\varphi^{k+1}\right|_{\infty}\left|h^{k} \nabla u^{k}\right|_{\infty}\right), \quad S^{k}(t)=\left(\left|u_{t}^{k}\right|_{3}+\left|u^{k-1}\right|_{\infty}\left|\nabla u^{k-1}\right|_{3}\right), \\
M^{k}(t) & =\left(\left|f^{k}\right|_{\infty}+\left|f^{k-1}\right|_{\infty}\right)\left|h^{k-1} \operatorname{div} u^{k-1}\right|_{\infty} .
\end{aligned}
$$

Then for $\bar{\varphi}^{k+1}$, multiplying (3.94) 4 by $2 \bar{\varphi}^{k+1}$ and integrating over $\mathbb{R}^{3}$ lead to

$$
\begin{aligned}
\frac{d}{d t}\left|\bar{\varphi}^{k+1}\right|_{2}^{2} \leq & C R^{k}(t)\left|\bar{\varphi}^{k+1}\right|_{2}^{2}+C\left|\bar{u}^{k}\right|_{3}\left|\nabla \varphi^{k}\right|_{6}\left|\bar{\varphi}^{k+1}\right|_{2} \\
& +C\left(\left|\nabla \bar{u}^{k}\right|_{2}\left|\varphi^{k-1}\right|_{\infty}+\left|\bar{\varphi}^{k}\right|_{2}\left|\nabla u^{k}\right|_{\infty}\right)\left|\bar{\varphi}^{k+1}\right|_{2}+C R^{k-1}\left|\bar{\varphi}^{k}\right|_{2}\left|\bar{\varphi}^{k+1}\right|_{2} .
\end{aligned}
$$

Then, applying $\partial_{x}^{\zeta}(|\zeta|=1)$ to $(\underline{3.94})_{4}$, multiplying by $2 \partial_{x}^{\zeta} \bar{\varphi}^{k+1}$ and integrating over $\mathbb{R}^{3}$, one has

$$
\begin{aligned}
& \frac{d}{d t}\left|\partial_{x}^{\zeta} \bar{\varphi}^{k+1}\right|_{2}^{2} \\
\leq & C\left(R^{k}+\left|h^{k} \nabla u^{k}\right|_{6}\left|\nabla \varphi^{k+1}\right|_{6}+\left|\varphi^{k+1}\right|_{\infty}\left|h^{k} \nabla^{2} u^{k}\right|_{3}+\left|\nabla^{2} u^{k}\right|_{3}\right)\left|\nabla \bar{\varphi}^{k+1}\right|_{2}^{2} \\
& \left.+C\left(\left|\nabla \varphi^{k-1}\right|_{6}\left|\nabla \bar{u}^{k}\right|_{3}+\left|\bar{\varphi}^{k+1}\right|_{2}\left|\varphi^{k+1}\right|_{\infty}\left|\psi^{k}\right|_{\infty}\left|\nabla u^{k}\right|_{\infty}\right)\right)\left|\nabla \bar{\varphi}^{k+1}\right|_{2} \\
& +C\left(\left|\bar{u}^{k}\right|_{6}\left|\nabla^{2} \varphi^{k}\right|_{3}+\left|\nabla^{2} u^{k}\right|_{3}\left|\bar{\varphi}^{k}\right|_{6}+\left|\nabla u^{k}\right|_{\infty}\left|\nabla \bar{\varphi}^{k}\right|_{2}\right)\left|\nabla \bar{\varphi}^{k+1}\right|_{2} \\
& +C\left(\left|\nabla \bar{u}^{k}\right|_{3}\left|\nabla \varphi^{k-1}\right|_{6}+\left|\varphi^{k-1}\right|_{\infty}\left|\nabla^{2} \bar{u}^{k}\right|_{2}\right)\left|\nabla \bar{\varphi}^{k+1}\right|_{2} \\
& +C\left(R^{k-1}+\left|\varphi^{k}\right|_{\infty}\left|\psi^{k-1}\right|_{\infty}\left|\nabla u^{k-1}\right|_{3}+\left|\nabla^{2} u^{k-1}\right|_{3}\right)\left|\nabla \bar{\varphi}^{k}\right|_{2}\left|\nabla \bar{\varphi}^{k+1}\right|_{2} \\
& +C\left(\left|\varphi^{k}\right|_{\infty}\left|h^{k-1} \nabla^{2} u^{k-1}\right|_{3}+\left|\nabla \varphi^{k}\right|_{6}\left|h^{k-1} \nabla u^{k-1}\right|_{6}\right)\left|\nabla \bar{\varphi}^{k}\right|_{2}\left|\nabla \bar{\varphi}^{k+1}\right|_{2},
\end{aligned}
$$

which, along with (3.100), implies that for $t \in\left[0, T^{* *}\right]$

$$
\frac{d}{d t}\left\|\bar{\varphi}^{k+1}(t)\right\|_{1}^{2} \leq C \sigma^{-1}\left\|\bar{\varphi}^{k+1}(t)\right\|_{1}^{2}+\sigma\left(\left\|\nabla \bar{u}^{k}\right\|_{1}^{2}+\left\|\bar{\varphi}^{k}\right\|_{1}^{2}\right) .
$$

For $\bar{u}^{k+1}$, multiplying $(3.94)_{2}$ by $2 \bar{u}^{k+1}$ and integrating over $\mathbb{R}^{3}$ yield

$$
\begin{aligned}
& \frac{d}{d t}\left|\sqrt{\varphi}^{k+1} \bar{u}^{k+1}\right|_{2}^{2}+2 a \alpha\left|\nabla \bar{u}^{k+1}\right|_{2}^{2}+2 a(\alpha+\beta)\left|\operatorname{div} \bar{u}^{k+1}\right|_{2}^{2} \\
= & \int\left(\varphi_{t}^{k+1}\left|\bar{u}^{k+1}\right|^{2}-2 \varphi^{k+1}\left(u^{k} \cdot \nabla \bar{u}^{k}+\bar{u}^{k} \cdot \nabla u^{k-1}\right) \cdot \bar{u}^{k+1}\right) \\
& +2 \int\left(-\bar{\varphi}^{k+1}\left(u_{t}^{k}+u^{k-1} \cdot \nabla u^{k-1}\right)-\varphi^{k+1} \nabla \bar{\phi}^{k+1}-\bar{\varphi}^{k+1} \nabla \phi^{k}\right) \cdot \bar{u}^{k+1} \\
& +2 \int\left(f^{k+1} \cdot Q\left(\bar{u}^{k}\right)+\bar{f}^{k+1} \cdot Q\left(u^{k-1}\right)\right) \cdot \bar{u}^{k+1}=\sum_{j=1}^{9} J_{j} .
\end{aligned}
$$

Now the terms on the right-hand side of (3.102) can be estimated as follows:

$$
\begin{aligned}
J_{1}= & \int \varphi_{t}^{k+1}\left|\bar{u}^{k+1}\right|^{2} \leq C\left|u^{k}\right|_{\infty}\left|\varphi^{k+1}\right|_{\infty}^{\frac{1}{2}}\left|\nabla \bar{u}^{k+1}\right|_{2}\left|\sqrt{\varphi}^{k+1} \bar{u}^{k+1}\right|_{2} \\
& +C\left(\left|\nabla u^{k}\right|_{\infty}+\left|h^{k} \nabla u^{k}\right|_{\infty}\left|\varphi^{k+1}\right|_{\infty}\right)\left|\sqrt{\varphi}^{k+1} \bar{u}^{k+1}\right|_{2}^{2},
\end{aligned}
$$


and

$$
\begin{aligned}
J_{2}+J_{3} & =\int-2 \varphi^{k+1}\left(u^{k} \cdot \nabla \bar{u}^{k}+\bar{u}^{k} \cdot \nabla u^{k-1}\right) \cdot \bar{u}^{k+1} \\
& \leq C\left|\varphi^{k+1}\right|_{\infty}^{\frac{1}{2}}\left|\sqrt{\varphi}^{k+1} \bar{u}^{k+1}\right|_{2}\left(\left|\nabla \bar{u}^{k}\right|_{2}\left|u^{k}\right|_{\infty}+\left|\bar{u}^{k}\right|_{6}\left|\nabla u^{k-1}\right|_{3}\right), \\
J_{4}+J_{5} & =-2 \int \bar{\varphi}^{k+1}\left(u_{t}^{k}+u^{k-1} \cdot \nabla u^{k-1}\right) \cdot \bar{u}^{k+1} \\
& \leq C S^{k}(t)\left|\bar{\varphi}^{k+1}\right|_{2}\left|\bar{u}^{k+1}\right|_{6}, \\
J_{6}+J_{7} & =-2 \int\left(\varphi^{k+1} \nabla \bar{\phi}^{k+1}+\bar{\varphi}^{k+1} \nabla \phi^{k}\right) \cdot \bar{u}^{k+1} \\
& \leq C\left|\varphi^{k+1}\right|_{\infty}^{\frac{1}{2}}\left|\sqrt{\varphi}^{k+1} \bar{u}^{k+1}\right|_{2}\left|\nabla \bar{\phi}^{k+1}\right|_{2}+C\left|\bar{\varphi}^{k+1}\right|_{2}\left|\nabla \phi^{k}\right|_{3}\left|\bar{u}^{k+1}\right|_{6}, \\
J_{8}+J_{9} & =2 \int\left(f^{k+1} \cdot Q\left(\bar{u}^{k}\right)+\bar{f}^{k+1} \cdot Q\left(u^{k-1}\right)\right) \cdot \bar{u}^{k+1} \\
& =2 \int\left(\varphi^{k+1} \psi^{k+1} \cdot Q\left(\bar{u}^{k}\right)+\bar{f}^{k+1} \cdot Q\left(u^{k-1}\right)\right) \cdot \bar{u}^{k+1} \\
& \leq C\left|\varphi^{k+1}\right|_{\infty}^{\frac{1}{2}}\left|\sqrt{\varphi}^{k+1} \bar{u}^{k+1}\right|_{2}\left|\psi^{k+1}\right|_{\infty}\left|\nabla \bar{u}^{k}\right|_{2}+C\left|\bar{f}^{k+1}\right|_{2}\left|\nabla u^{k-1}\right|_{3}\left|\bar{u}^{k+1}\right|_{6},
\end{aligned}
$$

where one has used the equation $(3.93)$ for the term $\varphi_{t}^{k+1}$.

It then follows from (3.102)-(3.104) and Young's inequality that

$$
\begin{aligned}
& \frac{d}{d t}\left|\sqrt{\varphi}^{k+1} \bar{u}^{k+1}\right|_{2}^{2}+2 a \alpha\left|\nabla \bar{u}^{k+1}\right|_{2}^{2}+2 a(\alpha+\beta)\left|\operatorname{div} \bar{u}^{k+1}\right|_{2}^{2} \\
\leq & C \sigma^{-1}\left|\sqrt{\varphi}^{k+1} \bar{u}^{k+1}\right|_{2}^{2}+\sigma\left|\nabla \bar{u}^{k}\right|_{2}^{2}+C\left(\left\|\bar{\phi}^{k+1}\right\|_{1}^{2}+\left\|\bar{\varphi}^{k+1}\right\|_{1}^{2}+\left|\bar{f}^{k+1}\right|_{2}^{2}\right) .
\end{aligned}
$$

Next multiplying $(3.94)_{2}$ by $2 \bar{u}_{t}^{k+1}$ and integrating it over $\mathbb{R}^{3}$ show that

$$
\begin{aligned}
& 2\left|\sqrt{\varphi}^{k+1} \bar{u}_{t}^{k+1}\right|_{2}^{2}+\frac{d}{d t}\left(\alpha\left|\nabla \bar{u}^{k+1}\right|_{2}^{2}+(\alpha+\beta)\left|\operatorname{div} \bar{u}^{k+1}\right|_{2}^{2}\right) \\
= & 2 \int\left(-\varphi^{k+1}\left(u^{k} \cdot \nabla \bar{u}^{k}+\bar{u}^{k} \cdot \nabla u^{k-1}\right) \cdot \bar{u}_{t}^{k+1}\right) \\
& +2 \int\left(-\bar{\varphi}^{k+1}\left(u_{t}^{k}+u^{k-1} \cdot \nabla u^{k-1}\right)-\varphi^{k+1} \nabla \bar{\phi}^{k+1}-\bar{\varphi}^{k+1} \nabla \phi^{k}\right) \cdot \bar{u}_{t}^{k+1} \\
& +2 \int\left(f^{k+1} \cdot Q\left(\bar{u}^{k}\right)+\bar{f}^{k+1} \cdot Q\left(u^{k-1}\right)\right) \cdot \bar{u}_{t}^{k+1}=\sum_{j=10}^{17} J_{j} .
\end{aligned}
$$

Now the terms on the right-hand side of (3.106) admit the following estimates

$$
\begin{aligned}
& J_{10}+J_{11}=2 \int-\varphi^{k+1}\left(u^{k} \cdot \nabla \bar{u}^{k}+\bar{u}^{k} \cdot \nabla u^{k-1}\right) \cdot \bar{u}_{t}^{k+1} \\
& \quad \leq C\left|\varphi^{k+1}\right|_{\infty}^{\frac{1}{2}}\left|\sqrt{\varphi}{ }^{k+1} \bar{u}_{t}^{k+1}\right|_{2}\left(\left|\nabla \bar{u}^{k}\right|_{2}\left|u^{k}\right|_{\infty}+\left|\bar{u}^{k}\right|_{6}\left|\nabla u^{k-1}\right|_{3}\right),
\end{aligned}
$$


and

$$
\begin{aligned}
& J_{12}+J_{13}=-2 \int \bar{\varphi}^{k+1}\left(u_{t}^{k}+u^{k-1} \cdot \nabla u^{k-1}\right) \cdot \bar{u}_{t}^{k+1} \\
& =-2 \frac{d}{d t} \int \bar{\varphi}^{k+1}\left(u_{t}^{k}+u^{k-1} \cdot \nabla u^{k-1}\right) \cdot \bar{u}^{k+1} \\
& +2 \int\left(\bar{\varphi}^{k+1}\left(u_{t}^{k}+u^{k-1} \cdot \nabla u^{k-1}\right)\right)_{t} \cdot \bar{u}^{k+1} \\
& \leq-2 \frac{d}{d t} \int \bar{\varphi}^{k+1}\left(u_{t}^{k}+u^{k-1} \cdot \nabla u^{k-1}\right) \cdot \bar{u}^{k+1} \\
& +C\left|\bar{\varphi}^{k+1}\right|_{3}\left|\bar{u}^{k+1}\right|_{6}\left(\left|u_{t t}^{k}\right|_{2}+\left|u_{t}^{k-1}\right|_{2}\left|\nabla u^{k-1}\right|_{\infty}+\left|u^{k-1}\right|_{\infty}\left|\nabla u_{t}^{k-1}\right|_{2}\right) \\
& +C\left|\bar{u}^{k+1}\right|_{6}\left(\left|u^{k}\right|_{\infty}\left|\nabla \bar{\varphi}^{k+1}\right|_{2}+\left|\bar{u}^{k}\right|_{3}\left|\nabla \varphi^{k}\right|_{6}+\left|\nabla u^{k}\right|_{\infty}\left|\bar{\varphi}^{k}\right|_{2}\right) S^{k}(t) \\
& +C\left|\bar{u}^{k+1}\right|_{6}\left(\left|\bar{\varphi}^{k+1}\right|_{2} R^{k}(t)+\left|\bar{\varphi}^{k}\right|_{2} R^{k-1}(t)+\left|\varphi^{k-1}\right|_{\infty}\left|\nabla \bar{u}^{k}\right|_{2}\right) S^{k}(t), \\
& J_{14}=-2 \int \varphi^{k+1} \nabla \bar{\phi}^{k+1} \cdot \bar{u}_{t}^{k+1} \mathrm{~d} x \leq C\left|\varphi^{k+1}\right|_{\infty}^{\frac{1}{2}}\left|\sqrt{\varphi}^{k+1} \bar{u}_{t}^{k+1}\right|_{2}\left|\nabla \bar{\phi}^{k+1}\right|_{2} \text {, } \\
& J_{15}=-2 \int \bar{\varphi}^{k+1} \nabla \phi^{k} \cdot \bar{u}_{t}^{k+1}=-2 \frac{d}{d t} \int \bar{\varphi}^{k+1} \nabla \phi^{k} \cdot \bar{u}^{k+1} \\
& +2 \int \bar{\varphi}_{t}^{k+1} \nabla \phi^{k} \cdot \bar{u}^{k+1}+2 \int \bar{\varphi}^{k+1} \nabla \phi_{t}^{k} \cdot \bar{u}^{k+1} \\
& \leq-2 \frac{d}{d t} \int \bar{\varphi}^{k+1} \nabla \phi^{k} \cdot \bar{u}^{k+1} \mathrm{~d} x+C\left|\bar{\varphi}^{k+1}\right|_{3}\left|\nabla \phi_{t}^{k}\right|_{2}\left|\bar{u}^{k+1}\right|_{6} \\
& +C\left|\bar{u}^{k+1}\right|_{6}\left|\nabla \phi^{k}\right|_{3}\left(\left|\nabla \bar{\varphi}^{k+1}\right|_{2}\left|u^{k}\right|_{\infty}+\left|\bar{u}^{k}\right|_{2}\left|\nabla \phi^{k}\right|_{\infty}\right) \\
& +C\left|\bar{u}^{k+1}\right|_{6}\left|\nabla \phi^{k}\right|_{3}\left(\left|\bar{\varphi}^{k}\right|_{2}\left|\nabla u^{k}\right|_{\infty}+\left|\varphi^{k-1}\right|_{\infty}\left|\nabla \bar{u}^{k}\right|_{2}\right) \\
& +C\left|\bar{u}^{k+1}\right|_{6}\left|\nabla \phi^{k}\right|_{3}\left(\left|\bar{\varphi}^{k+1}\right|_{2} R^{k}(t)+\left|\bar{\varphi}^{k}\right|_{2} R^{k-1}(t)\right) \text {, } \\
& J_{16}=2 \int f^{k+1} \cdot Q\left(\bar{u}^{k}\right) \cdot \bar{u}_{t}^{k+1} \leq C\left|\varphi^{k+1}\right|_{\infty}^{\frac{1}{2}}\left|\sqrt{\varphi}^{k+1} \bar{u}_{t}^{k+1}\right|_{2}\left|\psi^{k+1}\right|_{\infty}\left|\nabla \bar{u}^{k}\right|_{2} \text {, } \\
& J_{17}=2 \int \bar{f}^{k+1} \cdot Q\left(u^{k-1}\right) \cdot \bar{u}_{t}^{k+1}=2 \frac{d}{d t} \int \bar{f}^{k+1} \cdot Q\left(u^{k-1}\right) \cdot \bar{u}^{k+1} \\
& -2 \int \bar{f}^{k+1} \cdot Q\left(u^{k-1}\right)_{t} \cdot \bar{u}^{k+1} \mathrm{~d} x-2 \int \bar{f}_{t}^{k+1} \cdot Q\left(u^{k-1}\right) \cdot \bar{u}^{k+1} \\
& \leq 2 \frac{d}{d t} \int \bar{f}^{k+1} \cdot Q\left(u^{k-1}\right) \cdot \bar{u}^{k+1} \mathrm{~d} x+C\left|\nabla \bar{u}^{k+1}\right|_{2}\left|\nabla \bar{u}^{k}\right|_{2}\left|\nabla u^{k-1}\right|_{\infty} \\
& +C\left|\nabla \bar{u}^{k+1}\right|_{2}\left|\nabla \bar{u}^{k}\right|_{2}\left|\nabla^{2} u^{k-1}\right|_{3}+C\left|\bar{f}^{k+1}\right|_{2}\left|\nabla \bar{u}^{k+1}\right|_{2}\left|\nabla u^{k}\right|_{6}\left|\nabla u^{k-1}\right|_{6} \\
& +C\left|\bar{f}^{k+1}\right|_{2}\left|\nabla \bar{u}^{k+1}\right|_{2}\left(\left|u^{k}\right|_{6}\left|\nabla^{2} u^{k-1}\right|_{6}+\left|u^{k}\right|_{\infty}\left|\nabla u^{k-1}\right|_{\infty}\right) \\
& +C\left|\bar{f}^{k+1}\right|_{2}\left|\bar{u}^{k+1}\right|_{6}\left(\left|\nabla u_{t}^{k-1}\right|_{3}+\left|\nabla u^{k}\right|_{6}\left|\nabla u^{k-1}\right|_{6}\right) \\
& +C\left|\bar{u}^{k+1}\right|_{6}\left|\nabla u^{k-1}\right|_{3}\left(\left|\bar{u}^{k}\right|_{6}\left|\nabla f^{k}\right|_{3}+\left|f^{k}\right|_{\infty}\left|\nabla \bar{u}^{k}\right|_{2}\right) \\
& +C\left(\left|\bar{\varphi}^{k+1}\right|_{2}\left|h^{k} \nabla \operatorname{div} u^{k}\right|_{\infty}+\left|\bar{\varphi}^{k}\right|_{2}\left|h^{k-1} \nabla \operatorname{div} u^{k-1}\right|_{\infty}\right)\left|\nabla u^{k-1}\right|_{3}\left|\bar{u}^{k+1}\right|_{6} \\
& +C\left|\nabla u^{k-1}\right|_{3}\left|\bar{u}^{k+1}\right|_{6}\left(\left|\nabla u^{k}\right|_{\infty}\left(\left|\bar{f}^{k}\right|_{2}+\left|\bar{f}^{k+1}\right|_{2}\right)+\left(\left|f^{k}\right|_{\infty}+\left|f^{k-1}\right|_{\infty}\right)\left|\nabla \bar{u}^{k}\right|_{2}\right) \\
& +C\left(M^{k}(t)\left|\bar{\varphi}^{k}\right|_{2}+M^{k+1}(t)\left|\bar{\varphi}^{k+1}\right|_{2}\right)\left|\nabla u^{k-1}\right|_{\infty}\left|\bar{u}^{k+1}\right|_{2} \text {, }
\end{aligned}
$$


where one has used the fact that

$$
\left\{\begin{array}{l}
\bar{f}_{t}^{k+1}=-\sum_{l=1}^{3} A_{l}\left(u^{k}\right) \partial_{l} \bar{f}^{k+1}-B^{*}\left(u^{k}\right) \bar{f}^{k+1}-a \delta \nabla \operatorname{div} \bar{u}^{k}+\Upsilon_{1}^{k}+\Upsilon_{2}^{k}+\Upsilon_{3}^{k} \\
\bar{\varphi}_{t}^{k+1}=-u^{k} \cdot \nabla \bar{\varphi}^{k+1}-\bar{u}^{k} \cdot \nabla \varphi^{k}-(1-\delta)\left(\bar{\varphi}^{k} \operatorname{div} u^{k}+\varphi^{k-1} \operatorname{div} \bar{u}^{k}+\Upsilon_{4}^{k}\right)
\end{array}\right.
$$

It follows from (3.106)-(3.108) and Young's inequality that

$$
\begin{aligned}
& \left|\sqrt{\varphi}^{k+1} \bar{u}_{t}^{k+1}\right|_{2}^{2}+\frac{d}{d t}\left(a \alpha\left|\nabla \bar{u}^{k+1}\right|_{2}^{2}+a(\alpha+\beta)\left|\operatorname{div} \bar{u}^{k+1}\right|_{2}^{2}\right) \\
\leq & J_{18}+E_{\sigma}^{k}(t)\left|\nabla \bar{u}^{k+1}\right|_{2}^{2}+C\left|\nabla \bar{u}^{k}\right|_{2}^{2}+\sigma\left(\left|\bar{\varphi}^{k}\right|_{2}^{2}+\left|\bar{f}^{k}\right|_{2}^{2}\right) \\
& +C\left(\left\|\bar{\phi}^{k+1}\right\|_{1}^{2}+\left\|\bar{\varphi}^{k+1}\right\|_{1}^{2}+\left|\bar{f}^{k+1}\right|_{2}^{2}\right)
\end{aligned}
$$

where

$$
J_{18}=-\frac{d}{d t} \int\left(\bar{\varphi}^{k+1}\left(u_{t}^{k}+u^{k-1} \cdot \nabla u^{k-1}+\nabla \phi^{k}\right)+\bar{f}^{k+1} \cdot Q\left(u^{k-1}\right)\right) \cdot \bar{u}^{k+1},
$$

and $E_{\sigma}^{k}(t)$ satisfies

$$
\int_{0}^{t} E_{\sigma}^{k}(s) \mathrm{d} s \leq C+C \sigma^{-1} t, \quad \text { for } \quad 0 \leq t \leq T^{* *} .
$$

Hence, (3.96)-(3.97), (3.101), (3.105), (3.109)-(3.110) and the Gronwall's inequality imply that

$$
\begin{aligned}
& \frac{d}{d t}\left(\left|\sqrt{\varphi}^{k+1} \bar{u}^{k+1}\right|_{2}^{2}+\left\|\bar{\phi}^{k+1}\right\|_{1}^{2}+\left\|\bar{\varphi}^{k+1}\right\|_{1}^{2}+\left|\bar{f}^{k+1}\right|_{2}^{2}\right. \\
& \left.+a \alpha \nu\left|\nabla \bar{u}^{k+1}\right|_{2}^{2}+a(\alpha+\beta) \nu\left|\operatorname{div} \bar{u}^{k+1}\right|_{2}^{2}\right)+\left(a \alpha\left|\nabla \bar{u}^{k+1}\right|_{2}^{2}+\nu\left|\sqrt{\varphi}^{k+1} \bar{u}_{t}^{k+1}\right|_{2}^{2}\right) \\
\leq & \nu J_{18}+\left(N_{\sigma}^{k}(t)+\nu\right)\left(\left|\sqrt{\varphi}^{k+1} \bar{u}^{k+1}\right|_{2}^{2}+a \alpha\left|\nabla \bar{u}^{k+1}\right|_{2}^{2}+\left\|\bar{\phi}^{k+1}\right\|_{1}^{2}+\left\|\bar{\varphi}^{k+1}\right\|_{1}^{2}+\left|\bar{f}^{k+1}\right|_{2}^{2}\right) \\
& +C(\nu+\sigma) a \alpha\left|\nabla \bar{u}^{k}\right|_{2}^{2}+C \sigma\left(\left\|\bar{\phi}^{k}\right\|_{1}^{2}+\left|\nabla^{2} \bar{u}^{k}\right|_{2}^{2}+(1+\nu)\left(\left\|\bar{\varphi}^{k}\right\|_{1}^{2}+\left|\bar{f}^{k}\right|_{2}^{2}\right)\right),
\end{aligned}
$$

where $\nu \in(0,1)$ is a sufficiently small constant, and $N_{\epsilon}^{k}(t)$ satisfies

$$
\int_{0}^{t} N_{\sigma}^{k}(s) \mathrm{d} s \leq C+C \sigma^{-1} t, \quad \text { for } \quad 0 \leq t \leq T^{* *} .
$$

For $J_{18}$, one has

$$
\int_{0}^{t} J_{18} \mathrm{~d} s \leq C\left(\left|\bar{u}^{k+1}\right|_{2}^{2}+\left\|\bar{\varphi}^{k+1}\right\|_{1}^{2}+\left|\bar{f}^{k+1}\right|_{2}^{2}\right) .
$$

For the term $\nabla^{2} \bar{u}^{k}$, according to equations (3.94) 2 and Lemma 2.5, one has

$$
\left|\bar{u}^{k}\right|_{D^{2}} \leq C\left(\left|\sqrt{\varphi} \bar{u}_{t}^{k}\right|_{2}+\left|\nabla \bar{u}^{k-1}\right|_{2}+\left\|\bar{\phi}^{k}\right\|_{1}+\left\|\bar{\varphi}^{k}\right\|_{1}+\left|\bar{f}^{k}\right|_{2}\right) .
$$

Finally, define

$$
\begin{aligned}
\Gamma^{k+1}(t, \nu)= & \sup _{0 \leq s \leq t}\left\|\bar{\phi}^{k+1}(s)\right\|_{1}^{2}+\sup _{0 \leq s \leq t}\left\|\bar{\varphi}^{k+1}(s)\right\|_{1}^{2}+\sup _{0 \leq s \leq t}\left|\bar{f}^{k+1}(s)\right|_{2}^{2} \\
& +\sup _{0 \leq s \leq t}\left|\sqrt{\varphi}^{k+1} \bar{u}^{k+1}(s)\right|_{2}^{2}+a \nu \sup _{0 \leq s \leq t}\left(\alpha\left|\nabla \bar{u}^{k+1}(s)\right|_{2}^{2}+(\alpha+\beta)\left|\operatorname{div} \bar{u}^{k+1}(s)\right|_{2}^{2}\right) .
\end{aligned}
$$


Then it follows from (3.111)-(3.114) that

$$
\begin{aligned}
& \Gamma^{k+1}(t, \nu)+\int_{0}^{t}\left(a \alpha\left|\nabla \bar{u}^{k+1}\right|_{2}^{2}+\nu\left|\sqrt{\varphi}^{k+1} \bar{u}_{t}^{k+1}\right|_{2}^{2}\right) \mathrm{d} s \\
\leq & C\left(\int_{0}^{t}\left(a \alpha(\sigma+\nu)\left(\left|\nabla \bar{u}^{k}\right|_{2}^{2}+\left|\nabla \bar{u}^{k-1}\right|_{2}^{2}\right)+\sigma\left|\sqrt{\varphi}^{k} \bar{u}_{t}^{k}\right|_{2}^{2}\right) \mathrm{d} s+\sigma t \Gamma^{k}(t, \nu)\right) \exp \left(C+C \sigma^{-1} t\right) .
\end{aligned}
$$

Now, choose $\nu=\nu_{0} \in(0,1), \sigma=\sigma_{0} \in(0,1)$, and $T_{0}>0$ consecutively so that

$$
\begin{gathered}
C \nu_{0} \exp C \leq \frac{1}{32}, \\
C \sigma_{0} \exp C \leq \frac{\nu_{0}}{32}, \\
\left(T_{*}+1\right) \exp \left(C \sigma_{0}^{-1} T_{*}\right) \leq 4 .
\end{gathered}
$$

Then one gets easily

$$
\sum_{k=1}^{\infty}\left(\Gamma^{k+1}\left(T_{*}, \nu_{0}\right)+\int_{0}^{T_{*}}\left(a \alpha\left|\nabla \bar{u}^{k+1}\right|_{2}^{2}+\nu_{0}\left|\sqrt{\varphi}^{k+1} \bar{u}_{t}^{k+1}\right|_{2}^{2}\right) \leq C<+\infty .\right.
$$

Thanks to (3.115) and the local estimates (3.80) independent of $k$, one has

$$
\lim _{k \mapsto+\infty}\left|\bar{f}^{k+1}\right|_{6}+\lim _{k \mapsto+\infty}\left|\bar{\varphi}^{k+1}\right|_{\infty}=0 .
$$

Thus, by (3.115)-(3.116), one concludes that the whole sequence $\left(\phi^{k}, u^{k}, f^{k}, \varphi^{k}\right)$ converges to a limit $(\phi, u, f, \varphi)$ in the following strong sense: for any $s^{\prime} \in[1,3)$,

$$
\begin{aligned}
& \phi^{k}-\phi^{\infty} \rightarrow \phi-\phi^{\infty} \text { in } L^{\infty}\left(\left[0, T_{*}\right] ; H^{s^{\prime}}\left(\mathbb{R}^{3}\right)\right), \quad f^{k} \rightarrow f \text { in } L^{\infty}\left(\left[0, T_{*}\right] ; L^{6}\left(\mathbb{R}^{3}\right)\right), \\
& \varphi^{k} \rightarrow \varphi \text { in } L^{\infty}\left(\left[0, T_{*}\right] ; L^{\infty}\left(\mathbb{R}^{3}\right)\right), \quad u^{k} \rightarrow u \text { in } L^{\infty}\left(\left[0, T_{*}\right] ; D^{1} \cap D^{s^{\prime}}\left(\mathbb{R}^{3}\right)\right) .
\end{aligned}
$$

Again by virtue of the local estimates (3.80) independent of $k$, there exists a subsequence (still denoted by $\left.\left(\phi^{k}, u^{k}, f^{k}, \varphi^{k}, \psi^{k}\right)\right)$ converging to the limit $(\phi, u, f, \varphi, \psi)$ in the weak or weak* sense. According to the lower semi-continuity of norms, the corresponding estimates in (3.80) for $(\phi, u, f, \varphi, \psi)$ still hold except those weighted estimates on $u$. Thus, $(\phi, u, f, \varphi)$ is a weak solution in the sense of distributions to the following Cauchy problem:

$$
\left\{\begin{array}{l}
\phi_{t}+u \cdot \nabla \phi+(\gamma-1) \phi \operatorname{div} u=0 \\
\varphi\left(u_{t}+u \cdot \nabla u+\nabla \phi\right)=-a L u+f \cdot Q(u), \\
f_{t}+\sum_{l=1}^{3} A_{l}(u) \partial_{l} f+B^{*}(u) f+a \delta \nabla \operatorname{div} u=0 \\
\varphi_{t}+u \cdot \nabla \varphi-(\delta-1) \varphi \operatorname{div} u=0 \\
\left.(\phi, u, f, \varphi)\right|_{t=0}=\left(\phi_{0}, u_{0}, \phi_{0}^{-2 e} \psi_{0}, \phi_{0}^{-2 e}\right) \\
(\phi, u, f, \varphi) \rightarrow\left(\phi^{\infty}, 0,0,\left(\phi^{\infty}\right)^{-2 e}\right), \text { as }|x| \rightarrow+\infty, t \geq 0
\end{array}\right.
$$


Step 1.2: Strong convergence of $\psi^{k}$ and the existence to the problem (3.89). However, the conclusions obtained in Step 1.1 are still insufficient to show the desired existence of the strong solution to the Cauchy problem (3.89).

For this purpose, one first needs to check the strong convergence of $\psi^{k}$ :

$$
\left|\psi^{k+1}-\psi^{k}\right|_{6}=\left|\frac{f^{k+1} \varphi^{k}-f^{k} \varphi^{k+1}}{\varphi^{k+1} \varphi^{k}}\right|_{6} \leq C\left(\left|\varphi^{k}\right|_{\infty}\left|\bar{f}^{k+1}\right|_{6}+\left|f^{k}\right|_{6}\left|\bar{\varphi}^{k+1}\right|_{\infty}\right),
$$

which, along with (3.117), implies that

$$
\psi^{k} \rightarrow \psi \text { in } L^{\infty}\left(\left[0, T_{*}\right] ; L^{6}\left(\mathbb{R}^{3}\right)\right) .
$$

Next, one needs to show the relation $f=\psi \varphi$ still holds for the limit functions. Due to

$$
\left|f^{k}-\psi \varphi\right|_{6} \leq C\left(\left|\varphi^{k}-\varphi\right|_{\infty}\left|\psi^{k}\right|_{6}+\left.\left.\left|\psi^{k}-\psi\right|_{6}\right|_{\varphi}\right|_{\infty}\right) \rightarrow 0, \quad \text { as } \quad k \rightarrow \infty,
$$

then it holds that

$$
f(t, x)=\psi \varphi(t, x), \quad \text { a.e. } \quad \text { on } \quad\left[0, T_{*}\right] \times \mathbb{R}^{3} .
$$

Next to vertify the relations

$$
f=\frac{2 a e \delta}{\delta-1} \frac{\nabla \phi}{\phi}, \quad \varphi=\phi^{-2 e} \quad \text { and } \quad \psi=\frac{a \delta}{\delta-1} \nabla \phi^{2 e}
$$

we denote

$$
f^{*}=f-\frac{2 a e \delta}{\delta-1} \frac{\nabla \phi}{\phi} \quad \text { and } \quad \varphi^{*}=\varphi-\phi^{-2 e} .
$$

Then it follows from the equations $(3.118)_{1}$ and $(3.118){ }_{3}-(3.118) 4$ that

$$
\left\{\begin{array}{l}
f_{t}^{*}+\sum_{l=1}^{3} A_{l}(u) \partial_{l} f^{*}+B^{*}(u) f^{*}=0, \\
\varphi_{t}^{*}+u \cdot \nabla \varphi^{*}-(\delta-1) \varphi^{*} \operatorname{div} u=0, \\
\left.\left(f^{*}, \varphi^{*}\right)\right|_{t=0}=(0,0), \\
\left(f^{*}, \varphi^{*}\right) \rightarrow(0,0), \text { as }|x| \rightarrow+\infty, t \geq 0
\end{array}\right.
$$

which, together with a standard energy method, implies that

$$
f^{*}=0 \quad \text { and } \quad \varphi^{*}=0 \quad \text { for } \quad(t, x) \in\left[0, T_{*}\right] \times \mathbb{R}^{3} .
$$

Then the first two relations in (3.123) have been verified, and the last one follows easily from the relation (3.122).

Moreover, denoting $h=\varphi^{-1}$, one needs to show the following weak convergence:

$$
h^{k} \nabla^{2} u^{k} \rightarrow \varphi^{-1} \nabla^{2} u \quad \text { weakly* in } L^{\infty}\left(\left[0, T_{*}\right] ; H^{1}\right) \cap L^{2}\left(\left[0, T_{*}\right] ; D^{1}\right) .
$$


Indeed, due to the uniform positivity for $\varphi^{k} \geq \underline{\eta}>0$ and $\varphi \geq \underline{\eta}$, (3.117), and the upper bounds of the norms of $(\phi, u, \varphi, f)$, one has

$$
\begin{aligned}
& \int_{0}^{T_{*}} \int_{\mathbb{R}^{3}}\left(h^{k} \nabla^{2} u^{k}-\varphi^{-1} \nabla^{2} u\right) w \mathrm{~d} x \mathrm{~d} t \\
= & \int_{0}^{T_{*}} \int_{\mathbb{R}^{3}}\left(\left(\frac{\varphi-\varphi^{k}}{\varphi^{k} \varphi}\right) \nabla^{2} u^{k}+\varphi^{-1}\left(\nabla^{2} u^{k}-\nabla^{2} u\right)\right) w \mathrm{~d} x \mathrm{~d} t \\
\leq & C(\underline{\eta})\left(\sup _{0 \leq t \leq T_{*}}\left|\varphi^{k}-\varphi\right|_{\infty}+\left\|\nabla^{2} u^{k}-\nabla^{2} u\right\|_{L^{\infty}\left(\left[0, T_{*}\right] ; L^{2}\right)}\right) T_{*} \rightarrow 0, \quad \text { as } \quad k \rightarrow+\infty
\end{aligned}
$$

for any test functions $w(t, x) \in \mathbb{R}^{3}$ and $w(t, x) \in C_{c}^{\infty}\left(\left[0, T^{*}\right) \times \mathbb{R}^{3}\right)$, which implies that

$$
h^{k} \nabla^{2} u^{k} \rightarrow \varphi^{-1} \nabla^{2} u \quad \text { weakly* in } \in L^{\infty}\left(\left[0, T_{*}\right] ; H^{1}\right) .
$$

Similarly, one can also obtain that

$$
\begin{array}{rll}
\sqrt{h^{k}}\left(\nabla u^{k}, \nabla u_{t}^{k}\right)-\sqrt{h}\left(\nabla u, \nabla u_{t}\right) & \text { weakly* } \text { in } \quad L^{\infty}\left(\left[0, T^{*}\right] ; L^{2}\right), \\
h^{k} \nabla^{2} u^{k} \rightarrow \varphi^{-1} \nabla^{2} u & \text { weakly in } \quad L^{2}\left(\left[0, T_{*}\right] ; D^{1} \cap D^{2}\right), \\
\left(h^{k} \nabla^{2} u^{k}\right)_{t} \rightarrow\left(\varphi^{-1} \nabla^{2} u\right)_{t} & \text { weakly in } \quad L^{2}\left(\left[0, T_{*}\right] ; L^{2}\right) .
\end{array}
$$

Then the corresponding weighted estimates for $u$ shown in the a priori estimates (3.80) still hold for the limit functions.

Based on the estimates (3.80), strong convergences shown in (3.117), (3.120), (3.126)(3.127) and relations (3.122)-(3.123), it is obvious that functions

$$
\left(\phi, u, h=\phi^{2 e}, \psi=\frac{a \delta}{\delta-1} \nabla \phi^{2 e}\right)
$$

satisfy (3.89) in the sense of distributions. The a priori estimates (3.80) hold for $(\phi, u, h)$, and

$$
\begin{aligned}
& \phi-\phi^{\infty} \in L^{\infty}\left(\left[0, T_{*}\right] ; H^{3}\right), \quad \psi \in L^{\infty}\left(\left[0, T_{*}\right] ; D^{1} \cap D^{2}\right), \\
& \frac{2}{3} \eta^{-2 e}<\varphi \in L^{\infty} \cap D^{1,6} \cap D^{2,3} \cap D^{3}, \quad f \in L^{\infty} \cap L^{6} \cap D^{1,3} \cap D^{2}, \\
& u \in L^{\infty}\left(\left[0, T_{*}\right] ; H^{3}\right) \cap L^{2}\left(\left[0, T_{*}\right] ; H^{4}\right), \quad u_{t} \in L^{\infty}\left(\left[0, T_{*}\right] ; H^{1}\right) \cap L^{2}\left(\left[0, T_{*}\right] ; D^{2}\right), \\
& u_{t t} \in L^{2}\left(\left[0, T_{*}\right] ; L^{2}\right), \quad t^{\frac{1}{2}} u \in L^{\infty}\left(\left[0, T_{*}\right] ; D^{4}\right), \\
& t^{\frac{1}{2}} u_{t} \in L^{\infty}\left(\left[0, T_{*}\right] ; D^{2}\right) \cap L^{2}\left(\left[0, T_{*}\right] ; D^{3}\right), \quad t^{\frac{1}{2}} u_{t t} \in L^{\infty}\left(\left[0, T_{*}\right] ; L^{2}\right) \cap L^{2}\left(\left[0, T_{*}\right] ; D^{1}\right) .
\end{aligned}
$$

Step 2: Uniqueness. Let $\left(\phi_{1}, u_{1}, h_{1}\right)$ and $\left(\phi_{2}, u_{2}, h_{2}\right)$ be two strong solutions to the Cauchy problem (3.89) satisfying the uniform estimates in (3.80). Set

$$
\begin{aligned}
\varphi_{i} & =\left(h_{i}\right)^{-1}, \quad \psi_{i}=\frac{a \delta}{\delta-1} \nabla h_{i}, \quad f_{i}=\frac{a \delta}{\delta-1} \nabla h_{i} / h_{i}=\psi_{i} \varphi_{i} \quad \text { for } \quad i=1,2, \\
\bar{\phi} & =\phi_{1}-\phi_{2}, \quad \bar{u}=u_{1}-u_{2}, \quad \bar{f}=f_{1}-f_{2}, \quad \bar{\varphi}=\varphi_{1}-\varphi_{2} .
\end{aligned}
$$


Then it follows from (3.118) that

$$
\left\{\begin{array}{l}
\bar{\phi}_{t}+u^{1} \cdot \nabla \bar{\phi}+\bar{u} \cdot \nabla \phi_{2}+(\gamma-1)\left(\bar{\phi} \operatorname{div} u_{1}+\phi_{2} \operatorname{div} \bar{u}^{k}\right)=0 \\
\varphi_{1} \bar{u}_{t}+\varphi_{1} u_{1} \cdot \nabla \bar{u}+\varphi_{1} \nabla \bar{\phi}+L \bar{u} \\
=-\bar{\varphi}\left(\left(u_{2}\right)_{t}+u_{2} \cdot \nabla u_{2}\right)-\varphi_{1} \bar{u} \cdot \nabla u_{2}-\bar{\varphi} \nabla \phi_{2}+f \cdot Q(\bar{u})+\bar{f} \cdot Q\left(u_{2}\right), \\
\bar{f}_{t}+\sum_{l=1}^{3} A_{l}\left(u_{1}\right) \partial_{l} \bar{f}+B^{*}\left(u_{1}\right) \bar{f}+a \delta \nabla \operatorname{div} \bar{u}=\bar{\Upsilon}_{1}+\bar{\Upsilon}_{2}, \\
\bar{\varphi}_{t}+u_{1} \cdot \nabla \bar{\varphi}+\bar{u} \cdot \nabla \varphi_{2}+(\delta-1)\left(\bar{\varphi} \operatorname{div} u_{1}+\varphi_{2} \operatorname{div} \bar{u}\right)=0
\end{array}\right.
$$

where $\bar{\Upsilon}_{1}$ and $\bar{\Upsilon}_{2}$ are defined as

$$
\bar{\Upsilon}_{1}=-\sum_{l=1}^{3}\left(A_{l}\left(u_{1}\right) \partial_{l} \psi_{2}-A_{l}\left(u_{2}\right) \partial_{l} \psi_{2}\right), \quad \bar{\Upsilon}_{2}=-\left(B\left(u_{1}\right) \psi_{2}-B\left(u_{2}\right) \psi_{2}\right) .
$$

Set

$$
\Phi(t)=\|\bar{\phi}(t)\|_{1}^{2}+\|\bar{\varphi}(t)\|_{1}^{2}+|\bar{f}(t)|_{2}^{2}+\left|\sqrt{\varphi}_{1} \bar{u}(t)\right|_{2}^{2}+|\nabla \bar{u}(t)|_{2}^{2} .
$$

In a similar way for the derivation of (3.96)-(3.111), one can show that

$$
\frac{d}{d t} \Phi(t)+C\left(|\nabla \bar{u}(t)|_{2}^{2}+\left|\sqrt{\varphi}_{1} \bar{u}_{t}\right|_{2}^{2}\right) \leq H(t) \Phi(t)
$$

where

$$
\int_{0}^{t} H(s) d s \leq C, \quad \text { for } \quad 0 \leq t \leq T_{*}
$$

It follows from the Gronwall's inequality that $\bar{\phi}=\bar{\varphi}=0$ and $\bar{f}=\bar{u}=0$. Thus the uniqueness is obtained.

Step 3. The time-continuity follows easily from the same procedure as in Lemma 3.1.

3.6. Taking limit from the non-vacuum flows to the flow with far field vacuum. Based on the local (in time) estimates in (3.80), now we are ready to prove Theorem 3.1 .

Proof. We divide the proof into four steps.

Step 1: The locally uniform positivity of $\phi$. For any $\eta \in(0,1)$, set

$$
\phi_{0}^{\eta}=\phi_{0}+\eta, \quad \psi_{0}^{\eta}=\frac{a \delta}{\delta-1} \nabla\left(\phi_{0}+\eta\right)^{2 e}, \quad h_{0}^{\eta}=\left(\phi_{0}+\eta\right)^{2 e} .
$$

Then the initial compatibility conditions can be given as

$$
\left\{\begin{array}{l}
\nabla u_{0}=\left(\phi_{0}+\eta\right)^{-e} g_{1}^{\eta}, \quad L u_{0}=\left(\phi_{0}+\eta\right)^{-2 e} g_{2}^{\eta} \\
\nabla\left(a\left(\phi_{0}+\eta\right)^{2 e} L u_{0}\right)=\left(\phi_{0}+\eta\right)^{-e} g_{3}^{\eta}
\end{array}\right.
$$


where $g_{i}^{\eta}(i=1,2,3)$ are given as

$$
\left\{\begin{array}{l}
g_{1}^{\eta}=\frac{\phi_{0}^{-e}}{\left(\phi_{0}+\eta\right)^{-e}} g_{1}, \quad g_{2}^{\eta}=\frac{\phi_{0}^{-2 e}}{\left(\phi_{0}+\eta\right)^{-2 e}} g_{2}, \\
g_{3}^{\eta}=\frac{\phi_{0}^{-3 e}}{\left(\phi_{0}+\eta\right)^{-3 e}}\left(g_{3}-\frac{a \eta \nabla \phi_{0}^{2 e}}{\phi_{0}+\eta} \phi_{0}^{e} L u_{0}\right) .
\end{array}\right.
$$

Then it follows from the the initial assumption (3.6)-(3.7) and (3.12) that there exists a $\eta_{1}>0$ such that if $0<\eta<\eta_{1}$, then

$$
\begin{aligned}
1+ & \eta+\left\|\phi_{0}^{\eta}-\eta\right\|_{3}+\left|\psi_{0}^{\eta}\right|_{D^{1} \cap D^{2}}+\left\|u_{0}\right\|_{3}+\left|g_{1}^{\eta}\right|_{2}+\left|g_{2}^{\eta}\right|_{2}+\left|g_{3}^{\eta}\right|_{2} \\
& +\left\|\left(h_{0}^{\eta}\right)^{-1}\right\|_{L^{\infty} \cap D^{1,6} \cap D^{2,3} \cap D^{3}}+\left\|\nabla h_{0}^{\eta} / h_{0}^{\eta}\right\|_{L^{\infty} \cap L^{6} \cap D^{1,3} \cap D^{2}} \leq \bar{c}_{0},
\end{aligned}
$$

where $\bar{c}_{0}$ is a positive constant independent of $\eta$. Therefore, for initial data $\left(\phi_{0}^{\eta}, u_{0}^{\eta}, \psi_{0}^{\eta}\right)$, the problem (3.89) admits a unique classical solution $\left(\phi^{\eta}, u^{\eta}, \psi^{\eta}\right)$ in $\left[0, T_{*}\right] \times \mathbb{R}^{3}$ satisfying the local estimates in (3.79)-(3.80) with $c_{0}$ replaced by $\bar{c}_{0}$, and the life span $T_{*}$ is also independent of $\eta$.

Moreover, the following property holds:

Lemma 3.9. For any $R_{0}>0$ and $\eta \in(0,1]$, there exists a constant $a_{R_{0}}$ such that

$$
\phi^{\eta}(t, x) \geq a_{R_{0}}>0, \quad \forall(t, x) \in\left[0, T_{*}\right] \times B_{R_{0}},
$$

where $a_{R}$ is independent of $\eta$.

Proof. It suffices to consider the case when $R_{0}$ is sufficiently large.

First, due to the initial assumptions on $\phi$ and $\psi$ :

$$
\phi_{0} \in H^{3}, \psi_{0}=\frac{\delta}{\delta-1}\left(\frac{A \gamma}{\gamma-1}\right)^{-2 e} \nabla \phi_{0}^{2 e} \in D^{1} \cap D^{2},
$$

one has $\nabla \phi_{0}^{2 e} \in L^{\infty}$. Therefore, the initial vacuum does not occur in the interior point but in the far field, and for every $R^{\prime}>2$, there exists a constant $C_{R^{\prime}}$ independent of $\eta$ such that

$$
\phi_{0}^{\eta}(x) \geq C_{R^{\prime}}+\eta>0, \quad \forall x \in B_{R^{\prime}} .
$$

Second, let $x\left(t ; x_{0}\right)$ be the particle path starting from $x_{0}$ at $t=0$, i.e.,

$$
\frac{d}{\mathrm{~d} t} x\left(t ; x_{0}\right)=u\left(t, x\left(t ; x_{0}\right)\right), \quad x\left(0 ; x_{0}\right)=x_{0} .
$$

Then denote by $B\left(t, R^{\prime}\right)$ the closed regions that are the images of $B_{R^{\prime}}$ under the flow map (3.134):

$$
B\left(t, R^{\prime}\right)=\left\{x\left(t ; x_{0}\right) \mid x_{0} \in B_{R^{\prime}}\right\}
$$

It follows from $(3.2)_{1}$ that

$$
\phi^{\eta}(t, x)=\phi_{0}^{\eta}\left(x_{0}\right) \exp \left(-\int_{0}^{t}(\gamma-1) \operatorname{div} u^{\eta}\left(s ; x\left(s ; x_{0}\right)\right) \mathrm{d} s\right) .
$$


According to (3.79)-(3.80), it holds that for $0 \leq t \leq T_{*}$,

$$
\begin{aligned}
& \int_{0}^{t} \mid \operatorname{div} u^{\eta}\left(t,\left.x\left(t ; x_{0}\right)\left|\mathrm{d} s \leq \int_{0}^{t}\right| \nabla u^{\eta}\right|_{\infty} \mathrm{d} s\right. \\
\leq & \int_{0}^{t}\left\|\nabla u^{\eta}\right\|_{2} \mathrm{~d} s \leq t^{\frac{1}{2}}\left(\int_{0}^{t}\left\|\nabla u^{\eta}\right\|_{2}^{2} \mathrm{~d} s\right)^{\frac{1}{2}} \leq c_{3} T_{*}^{\frac{1}{2}} .
\end{aligned}
$$

Thus, by (3.133) and (3.136), one can obtain that for $0 \leq t \leq T_{*}$,

$$
\phi^{\eta}(t, x) \geq C^{*}\left(C_{R^{\prime}}+\eta\right)>0, \quad \forall x \in B\left(t, R^{\prime}\right),
$$

where $C^{*}=\exp \left(-(\gamma-1) c_{3} T_{*}^{\frac{1}{2}}\right)$.

At last, it follows from (3.134) and (3.79)-(3.80) that

$$
\left|x_{0}-x\right|=\left|x_{0}-x\left(t ; x_{0}\right)\right| \leq \int_{0}^{t}\left|u^{\eta}\left(\tau, x\left(\tau ; x_{0}\right)\right)\right| \mathrm{d} \tau \leq c_{3} t \leq 1 \leq R^{\prime} / 2,
$$

for all $(t, x) \in\left[0, T_{*}\right] \times B_{R}$, which implies $B_{R^{\prime} / 2} \subset B\left(t, R^{\prime}\right)$. Thus, one can choose

$$
R^{\prime}=2 R_{0}, \quad \text { and } \quad a_{R_{0}}=C^{*} C_{R^{\prime}} .
$$

Step 2: Existence. First, since the estimates (3.80) are independent of $\eta$, then there exists a subsequence (still denoted by $\left.\left(\phi^{\eta}, u^{\eta}, \psi^{\eta}\right)\right)$ converging to a limit $(\phi, u, \psi)$ in weak or weak* sense:

$$
\begin{aligned}
\left(\phi^{\eta}-\eta, u^{\eta}\right) \rightarrow(\phi, u) & \text { weakly* in } L^{\infty}\left(\left[0, T_{*}\right] ; H^{3}\right), \\
u^{\eta} \rightarrow u & \text { weakly in } L^{2}\left(\left[0, T_{*}\right] ; H^{4}\right), \\
\varphi^{\eta} \rightarrow \varphi & \text { weakly* in } L^{\infty}\left(\left[0, T_{*}\right] ; L^{\infty} \cap D^{1,6} \cap D^{2,3} \cap D^{3}\right), \\
f^{\eta} \rightarrow f & \text { weakly* in } L^{\infty}\left(\left[0, T_{*}\right] ; L^{\infty} \cap L^{6} \cap D^{1,3} \cap D^{2}\right), \\
\psi^{\eta} \rightarrow \psi & \text { weakly* in } L^{\infty}\left(\left[0, T_{*}\right] ; D^{1} \cap D^{2}\right), \\
\phi_{t}^{\eta} \rightarrow \phi_{t} & \text { weakly* in } L^{\infty}\left(\left[0, T_{*}\right] ; H^{2}\right), \\
\left(u_{t}^{\eta}, \psi_{t}^{\eta}\right) \rightarrow\left(u_{t}, \psi_{t}\right) & \text { weakly* in } L^{\infty}\left(\left[0, T_{*}\right] ; H^{1}\right), \\
\varphi_{t}^{\eta} \rightarrow \varphi_{t} & \text { weakly* in } L^{\infty}\left(\left[0, T_{*}\right] ; L^{6} \cap D^{1,3} \cap D^{2}\right), \\
f_{t}^{\eta} \rightarrow f_{t} & \text { weakly* in } L^{\infty}\left(\left[0, T_{*}\right] ; L^{3} \cap D^{1}\right) .
\end{aligned}
$$

Then the lower semi-continuity of weak convergences implies that $(\phi, u, f, \varphi, \psi)$ satisfies the corresponding estimates (3.80) except those weighted estimates on $u$.

Second, for any $R>0$, due to the Aubin-Lions Lemma (see [26]) (i.e., Lemma 2.2), there exists a subsequence (still denoted by $\left.\left(\phi^{\eta}, u^{\eta}, \psi^{\eta}\right)\right)$ satisfying

$$
\begin{array}{rlrl}
\left(\phi^{\eta}-\eta, \psi^{\eta}, \varphi^{\eta}\right) & \rightarrow(\phi, \psi, \varphi) & & \text { in } C\left(\left[0, T_{*}\right] ; H^{1}\left(B_{R}\right)\right), \\
u^{\eta} \rightarrow u & & \text { in } C\left(\left[0, T_{*}\right] ; H^{2}\left(B_{R}\right)\right),
\end{array}
$$

where $B_{R}$ is a ball centered at origin with radius $R$.

Here, the following relations hold for the limit functions:

$$
f=\frac{2 a e \delta}{\delta-1} \frac{\nabla \phi}{\phi}, \quad \varphi=\phi^{-2 e} \quad \text { and } \quad \psi=\frac{a \delta}{\delta-1} \nabla \phi^{2 e}
$$


which can be proved by the same argument used in the proof of (3.123).

Let $h=\varphi^{-1}$. According to estimates (3.80) except those weighted terms of $u$, Lemma [3.9, the weak or weak* convergences shown in (3.138) and the strong convergences shown in (3.139), in a similar way for proving (3.126), one can obtain that

$$
\begin{aligned}
& \sqrt{h^{\eta}}\left(\nabla u^{\eta}, \nabla u_{t}^{\eta}\right) \rightarrow \sqrt{h}\left(\nabla u, \nabla u_{t}\right) \quad \text { weakly* in } L^{\infty}\left(\left[0, T^{*}\right] ; L^{2}\right), \\
& h^{\eta} \nabla^{2} u^{\eta} \rightarrow h \nabla^{2} u \quad \text { weakly* in } L^{\infty}\left(\left[0, T_{*}\right] ; H^{1}\right), \\
& h^{\eta} \nabla^{2} u^{\eta} \rightarrow h \nabla^{2} u \quad \text { weakly in } L^{2}\left(\left[0, T_{*}\right] ; D^{1} \cap D^{2}\right) \text {, } \\
& \left(h^{\eta} \nabla^{2} u^{\eta}\right)_{t} \rightarrow\left(h \nabla^{2} u\right)_{t} \quad \text { weakly in } L^{2}\left(\left[0, T_{*}\right] ; L^{2}\right) \text {. }
\end{aligned}
$$

Then the corresponding weighted estimates for $u$ shown in the a priori estimates (3.80) hold also for the limit functions.

Thus it is easy to show that $(\phi, u, \psi)$ solves the Cauchy problem (3.2)-(3.5) in the sense of distributions. Moreover, in this step, even though vacuum appears in the far field, $\psi$ satisfies $\partial_{i} \psi^{(j)}=\partial_{j} \psi^{(i)}(i, j=1,2,3)$ and solves the following positive symmetric hyperbolic system in the sense of distributions:

$$
\psi_{t}+\sum_{l=1}^{3} A_{l} \partial_{l} \psi+B \psi+\delta a \phi^{2 e} \nabla \operatorname{div} u=0 .
$$

Step 3. The uniqueness follows easily from the same procedure as that for Theorem 3.2 .

Step 4: Time continuity. The time continuity of $\phi$ and $\psi$ can be obtained via the similar arguments as used in Lemma 3.1.

For the velocity $u$, the a priori estimates obtained above and Sobolev's imbedding theorem imply that

$$
u \in C\left(\left[0, T_{*}\right] ; H^{2}\right) \cap C\left(\left[0, T_{*}\right] ; \text { weak- } H^{3}\right) \quad \text { and } \quad \phi^{e} \nabla u \in C\left(\left[0, T_{*}\right] ; L^{2}\right) .
$$

Then equations $(3.2)_{3}$ yield

$$
\varphi u_{t} \in L^{2}\left(\left[0, T_{*}\right] ; H^{2}\right), \quad\left(\varphi u_{t}\right)_{t} \in L^{2}\left(\left[0, T_{*}\right] ; L^{2}\right),
$$

which implies that $\varphi u_{t} \in C\left(\left[0, T_{*}\right] ; H^{1}\right)$. Together with classical elliptic estimates and

$$
a L u=-\varphi\left(u_{t}+u \cdot \nabla u+\nabla \phi-\psi \cdot Q(u)\right),
$$

one gets $u \in C\left(\left[0, T_{*}\right] ; H^{3}\right)$ immediately.

Next for $h \nabla^{2} u$, due to

$$
h \nabla^{2} u \in L^{\infty}\left(\left[0, T_{*}\right] ; H^{1}\right) \cap L^{2}\left(\left[0, T_{*}\right] ; D^{2}\right) \quad \text { and } \quad\left(h \nabla^{2} u\right)_{t} \in L^{2}\left(\left[0, T_{*}\right] ; L^{2}\right),
$$

and the classical Sobolev imbedding theorem, one gets quickly that

$$
h \nabla^{2} u \in C\left(\left[0, T_{*}\right] ; H^{1}\right) .
$$

Then the time continuity of $u_{t}$ follows easily. 
3.7. The proof for Theorem 1.1. With Theorem 3.1 at hand, now we are ready to establish the local-in-time well-posedness of the regular solution to the original Cauchy problem (1.1)-(1.7) shown in Theorem 1.1 .

Proof. The proof is divided into two steps.

Step 1. It follows from the initial assumptions (1.12)-(1.13) and Theorem 3.1 that there exists a time $T_{*}>0$ such that the problem (3.2)-(3.5) has a unique regular solution $(\phi, u, \psi)$ satisfying the regularity (3.8), which implies that

$$
\phi \in C^{1}\left(\left[0, T_{*}\right] \times \mathbb{R}^{3}\right), \quad(u, \nabla u) \in C\left(\left(0, T_{*}\right] \times \mathbb{R}^{3}\right) .
$$

Set $\rho=\left(\frac{\gamma-1}{A \gamma} \phi\right)^{\frac{1}{\gamma-1}}$ with $\rho(0, x)=\rho_{0}$. It follows from the proof of Theorem 3.1 (in particular, the proofs of (3.123) and (3.140) ) that the following relations hold:

$$
f=\frac{2 a e \delta}{\delta-1} \frac{\nabla \phi}{\phi}, \quad \varphi=\phi^{-2 e} \quad \text { and } \quad \psi=\frac{a \delta}{\delta-1} \nabla \phi^{2 e},
$$

which implies that

$$
f=a \delta \nabla \log \rho, \quad \varphi=a \rho^{1-\delta} \quad \text { and } \quad \psi=\frac{\delta}{\delta-1} \nabla \rho^{\delta-1} .
$$

Due to the above regularities and relations of the solution $(\phi, u, \psi)$, then multiplying (3.2) 1 by

$$
\frac{\partial \rho}{\partial \phi}(t, x)=\frac{1}{\gamma-1}\left(\frac{\gamma-1}{A \gamma}\right)^{\frac{1}{\gamma-1}} \phi^{\frac{2-\gamma}{\gamma-1}}(t, x),
$$

yields the continuity equation in (1.1), while multiplying (3.2) 2 by $\rho(t, x)$ gives the momentum equations in (1.1).

Thus we have shown that $(\rho, u)$ satisfies problem (1.1)-(1.7) in the sense of distributions and has the regularities shown in Definition 1.1 and (1.14). Finally, $\rho(t, x)>0$ for $(t, x) \in$ $\left[0, T_{*}\right] \times \mathbb{R}^{3}$ follows from the continuity equation.

In summary, the Cauchy problem (1.1)-(1.7) has a unique regular solution $(\rho, u)$.

Step 3. Now we show that, if $\gamma \in(1,2]$, the regular solution obtained in the above step is indeed a classical one within its life span.

First, due to $1<\gamma \leq 2$, one has

$$
\left(\rho, \nabla \rho, \rho_{t}, u, \nabla u\right) \in C\left(\left[0, T_{*}\right] \times \mathbb{R}^{3}\right) .
$$

Second, it follows from the classical Sobolev embedding result:

$$
L^{2}\left([0, T] ; H^{1}\right) \cap W^{1,2}\left([0, T] ; H^{-1}\right) \hookrightarrow C\left([0, T] ; L^{2}\right),
$$

and the regularity (1.14) that

$$
t u_{t} \in C\left(\left[0, T_{*}\right] ; H^{2}\right), \quad \text { and } \quad u_{t} \in C\left([\tau, T] \times \mathbb{R}^{3}\right) .
$$

Finally, it remains to show that $\nabla^{2} u \in C\left(\left[\tau, T_{*}\right] \times \mathbb{R}^{3}\right)$. According to Step 1 above, the following elliptic system holds

$$
a L u=-\phi^{-2 e}\left(u_{t}+u \cdot \nabla u+\nabla \phi-\psi \cdot Q(u)\right)=\phi^{-2 e} \mathbb{M} .
$$

The regularity (1.14) implies that

$$
t \phi^{-2 e} \mathbb{M} \in L^{\infty}\left(\left[0, T_{*}\right] ; H^{2}\right) .
$$


Note that

$$
\left(t \phi^{-2 e} \mathbb{M}\right)_{t}=\phi^{-2 e} \mathbb{M}+t \phi_{t}^{-2 e} \mathbb{M}+t \phi^{-2 e} \mathbb{M}_{t} \in L^{2}\left(\left[0, T_{*}\right] ; L^{2}\right) .
$$

So it follows from the classical Sobolev imbedding theorem:

$$
L^{\infty}\left([0, T] ; H^{1}\right) \cap W^{1,2}\left([0, T] ; H^{-1}\right) \hookrightarrow C\left([0, T] ; L^{q}\right),
$$

for any $q \in[2,6)$, and (3.148)-(3.150) that

$$
t \phi^{-2 e} \mathbb{M} \in C\left(\left[0, T_{*}\right] ; W^{1,4}\right), \quad t \nabla^{2} u \in C\left(\left[0, T_{*}\right] ; W^{1,4}\right) .
$$

Again the Sobolev embedding theorem implies that $\nabla^{2} u \in C\left(\left(0, T_{*}\right] \times \mathbb{R}^{3}\right)$.

Theorem 1.2 can be proved by the similar argument used in Theorem 1.1 under some slight modifications, so the details are omitted.

\section{Non-EXistence of Global SOlutions With $L^{\infty}$ DECAY ON $u$}

4.1. Proof of Theorem 1.3. Now we are ready to prove Theorem 1.3. Let $T>0$ be any constant, and $(\rho, u) \in D(T)$. It follows from the definitions of $m(t), \mathbb{P}(t)$ and $E_{k}(t)$ that

$$
|\mathbb{P}(t)| \leq \int \rho(t, x)|u|(t, x) \leq \sqrt{2 m(t) E_{k}(t)},
$$

which, together with the definition of the solution class $D(T)$, implies that

$$
0<\frac{|\mathbb{P}(0)|^{2}}{2 m(0)} \leq E_{k}(t) \leq \frac{1}{2} m(0)|u(t)|_{\infty}^{2} \quad \text { for } \quad t \in[0, T]
$$

Then one obtains that there exists a positive constant $C_{u}=\frac{|\mathbb{P}(0)|}{m(0)}$ such that

$$
|u(t)|_{\infty} \geq C_{u} \quad \text { for } \quad t \in[0, T] .
$$

Thus one obtains the desired conclusion as shown in Theorem 1.3 .

4.2. Proof of Corollary 1.1. Let $(\rho, u)(t, x)$ in $[0, T] \times \mathbb{R}^{3}$ be the regular solution defined in Definition 1.1. Next we just need to show that $(\rho, u) \in D(T)$.

First, it is easy to show that $(\rho, u)$ has finite total mass $m(t)$, finite momentum $\mathbb{P}(t)$, finite total energy $E(t)$.

Lemma 4.1. Let (1.11) and (1.19) hold, and $(\rho, u)$ be the regular solution defined in Definition 1.1, then

$$
m(t)+|\mathbb{P}(t)|+E(t)<+\infty \quad \text { for } \quad t \in[0, T] .
$$

Proof. Indeed, due to $1<\gamma \leq \frac{3}{2}$, one has

$$
\begin{array}{r}
m(t)=\int\left(\rho^{\gamma-1}\right)^{\frac{1}{\gamma-1}} \leq C\left|\rho^{\gamma-1}\right|_{2}^{2}<+\infty, \\
\mathbb{P}(t)=\int \rho u \leq C|\rho|_{1}|u|_{\infty}<+\infty, \\
E(t)=\int\left(\frac{1}{2} \rho|u|^{2}+\frac{P}{\gamma-1}\right) \leq C\left(|\rho|_{\infty}|u|_{2}^{2}+|\rho|_{\infty}^{\gamma-1}|\rho|_{1}\right)<+\infty .
\end{array}
$$


Second, the conservation of the total mass and momentum can be verified.

Lemma 4.2. Let (1.11) and (1.19) hold, and $(\rho, u)$ be the regular solution defined in Definition 1.1. Then

$$
\mathbb{P}(t)=\mathbb{P}(0) \quad \text { and } \quad m(t)=m(0) \quad \text { for } \quad t \in[0, T] .
$$

Proof. The momentum equations (1.1) 2 imply that

$$
\mathbb{P}_{t}=-\int \operatorname{div}(\rho u \otimes u)-\int \nabla P+\int \operatorname{div} \mathbb{T}=0
$$

where one has used the fact that

$$
\rho u^{(i)} u^{(j)}, \quad \rho^{\gamma} \quad \text { and } \quad \rho^{\delta} \nabla u \in W^{1,1}\left(\mathbb{R}^{3}\right) \quad \text { for } \quad i, j=1,2,3 .
$$

Similarly, we can show the conservation of the mass.

According to Theorem 1.3 and Lemmas 4.14.2, one obtains the desired conclusion in Corollary 1.1 .

Remark 4.1. First, under the assumptions of Corollary 1.1, the regular solution $(\rho, u)$ satisfies also the energy equality. Indeed, the continuity equation (1.1. $)_{1}$ and the definition of $P$ give

$$
P_{t}+u \cdot \nabla P+\gamma P \text { divu }=0
$$

While the momentum equations (1.1) 2 and (4.3) yield that

$$
\begin{aligned}
& E_{t}+\int \rho^{\delta}\left(\alpha|\nabla u|^{2}+(\alpha+\beta)|\operatorname{divu}|^{2}\right) \\
= & -\frac{1}{2} \int \operatorname{div}\left(\rho u|u|^{2}\right)-\frac{\gamma}{\gamma-1} \int \operatorname{div}(u P)+\int \operatorname{div}(u \mathbb{T}) .
\end{aligned}
$$

Due to the definition of the regular solution, (1.11) and (1.19), one gets that

$$
\rho u|u|^{2}, \quad u P \quad \text { and } \quad u \mathbb{T} \in W^{1,1}\left(\mathbb{R}^{3}\right),
$$

which, along with (4.4) implies the desired energy equality:

$$
E(t)+\int_{0}^{t} \int \rho^{\delta}\left(\alpha|\nabla u|^{2}+(\alpha+\beta)|d i v u|^{2}\right) d x d s=E(0) .
$$

Second, for the flows of constant viscosities [5, 10] $(\delta=0)$, it is not clear to verify the conservation of momentum for the strong solutions with vacuum for the Cauchy problem. The reason is that one is not sure whether $\mathbb{T} \in W^{1,1}\left(\mathbb{R}^{3}\right)$, or not.

4.3. Proof of Corollary 1.2. For the convenience of the proof, set:

$$
F \triangleq(2 \alpha+\beta) \operatorname{div} u-P(\rho), \quad \omega \triangleq \nabla \times u,
$$

where $F$ is the effective viscous flux, and $\omega$ is the vorticity.

Proof. The proof is divided into three steps: 
Step 1: Local-in-time well-posedness. It follows easily from the initial assumption (1.21) and arguments in [5, 6] that there exists a time $T_{0}>0$ and a unique classical solution $(\rho, u)$ in $\left(0, T_{0}\right) \times \mathbb{R}^{3}$ to the Cauchy problem (1.1)-(1.7) satisfying

$$
\begin{aligned}
& (\rho, P(\rho)) \in C\left(\left[0, T_{0}\right] ; H^{3}\right), \quad \rho^{\frac{1}{2}} \in C\left(\left[0, T_{0}\right] ; H^{1}\right), \\
& u \in C\left(\left[0, T_{0}\right] ; D^{1} \cap D^{3}\right) \cap L^{2}\left(\left[0, T_{0}\right] ; D^{4}\right), \quad u_{t} \in L^{\infty}\left(\left[0, T_{0}\right] ; D^{1}\right) \cap L^{2}\left(\left[0, T_{0}\right] ; D^{2}\right) .
\end{aligned}
$$

Step 2: Global-in-time well-posedness. It follows from the local-in-time well-posedenss obtained in Step 1, the smallness assumption (1.22) and the argument in [10] that the Cauchy problem (1.1)-(1.7) has a unique global classical solution $(\rho, u)$ in $(0, \infty) \times \mathbb{R}^{3}$ satisfying (1.23) and (1.25)-(1.28) for any $0<\tau<T<\infty$. It remains to show (1.24).

First, the continuity equation (1.1) 1 implies that $\rho^{\frac{1}{2}}$ satisfies the following equation

$$
\rho_{t}^{\frac{1}{2}}+u \cdot \nabla \rho^{\frac{1}{2}}+\frac{1}{2} \rho^{\frac{1}{2}} \operatorname{div} u=0
$$

which, along with the standard energy estimates argument for transport equations and (1.26), yields that

$$
\left\|\rho^{\frac{1}{2}}(t)\right\|_{1} \leq\left\|\rho_{0}^{\frac{1}{2}}\right\|_{1} \exp \left(C \int_{0}^{t}\|u\|_{D^{1} \cap D^{3}} \mathrm{~d} s\right)<\infty \quad \text { for } \quad 0<t \leq T,
$$

where $C>0$ is constant, and $T$ is any positive time. This, together with (4.6), shows

$$
\rho^{\frac{1}{2}} \in C\left([0, T] ; H^{1}\right) \text {. }
$$

Then (4.10) yields the conservation of the mass, since

$$
\frac{d}{d t} \int \rho=-\int \operatorname{div}(\rho u)=0, \quad \text { for } \quad 0<t \leq T
$$

due to $\rho u \in W^{1,1}\left(\mathbb{R}^{3}\right)$.

Step 3: Verification of the large time behavior on $u$. First, by the Gagliardo-Nirenberg inequality in Lemma 2.1, and Lemma 2.3 in [10], one has

$$
\begin{aligned}
|u|_{\infty} & \leq C|u|_{6}^{\frac{1}{2}}|u|_{6}^{\frac{1}{2}} \leq C|\nabla u|_{2}^{\frac{1}{2}}\left(|F|_{6}^{\frac{1}{2}}+|\omega|_{6}^{\frac{1}{2}}+|P|_{6}^{\frac{1}{2}}\right) \\
& \leq C|\nabla u|_{2}^{\frac{1}{2}}\left(|\rho \dot{u}|_{2}+|P|_{6}\right)^{\frac{1}{2}},
\end{aligned}
$$

which, together with (1.23), (1.25) and (1.28), implies that

$$
\limsup _{t \rightarrow+\infty}|u(t, x)|_{\infty}=0 \text {. }
$$

Finally, according to (4.12), and Theorem 1.3, if $m(0)>0$ and $|\mathbb{P}(0)|>0$, the global solution obtained in Step 2 above can not keep the conservation of momentum for all the time $t \in(0, \infty)$.

Conflict of Interest: The authors declare that they have no conflict of interest.

Acknowledgement: This research is partially supported by Zheng Ge Ru Foundation, Hong Kong RGC Earmarked Research Grants CUHK 14300917, CUHK-14305315 and CUHK 4048/13P, NSFC/RGC Joint Research Scheme Grant N-CUHK 443-14, and a Focus Area Grant from The Chinese University of Hong Kong. Zhu's research is also supported in part by National Natural Science Foundation of China under grant 11231006, Natural 
Science Foundation of Shanghai under grant 14ZR1423100, Australian Research Council grant DP170100630 and Newton International Fellowships NF170015.

\section{REFERENCES}

[1] J. Boldrini, M. Rojas-Medar and E. Fernández-Cara, Semi-Galerkin approximation and regular solutions to the equations of the nonhomogeneous asymmetric fluids, J. Math. Pure Appl. 82 (2003), 1499-1525.

[2] D. Bresch, B. Desjardins and C. Lin, On some compressible fluid models: Korteweg, Lubrication, and Shallow water systems, Commun. Part. Differ. Equations 28 (2003), 843-868.

[3] D. Bresch, B. Desjardins and G. Métivier, Recent mathematical results and open problems about shallow water equations, Anal. Simu. Fluid Dynam. (2006), 15-31.

[4] S. Chapman and T. Cowling, The mathematical theory of non-uniform gases: an account of the kinetic theory of viscosity, thermal conduction and diffusion in gases, Cambridge University Press, 1990.

[5] Y. Cho, H. Choe and H. Kim, Unique solvability of the initial boundary value problems for compressible viscous fluids, J. Math. Pure. Appl. 83 (2004), 243-275.

[6] Y. Cho and H. Kim, On classical solutions of the compressible Navier-Stokes equations with nonnegative initial densities, Manu. Math. 120 (2006), 91-129.

[7] M. Ding and S. Zhu, Vanishing viscosity limit of the Navier-Stokes equations to the Euler equations for compressible fluid flow with far field vacuum, J. Math. Pure Appl. 107 (2017), 288-314.

[8] G. Galdi, An introduction to the Mathmatical Theorey of the Navier-Stokes equations, Springer, New York, 1994.

[9] Y. Geng, Y. Li and S., Zhu, Vanishing viscosity limit of the Navier-Stokes equations to the Euler equations for compressible fluid flow with vacuum, submitted, 2015.

[10] X. Huang, J. Li and Z. Xin, Global well-posedness of classical solutions with large oscillations and vacuum to the Three-Dimensional Isentropic Compressible Navier-Stokes Equations, Comm. Pure. Appl. Math. 65 (2012), 549-585.

[11] Q. Jiu, Y. Wang and Z. Xin, Global well-posedness of 2D compressible Navier-Stokes equations with large data and vacuum, J. Math. Fluid Mech. 16 (2014), 483-521.

[12] S. Kawashima, Systems of a hyperbolic-parabolic composite type, with applications to the equations of magnetohydrodynamics, Ph.D thesis, Kyoto University, DOI: 10.14989/doctor.k3193, 1983.

[13] O. Ladyzenskaja and N. Ural'ceva, Linear and Quasilinear Equations of Parabolic Type, American Mathematical Society, Providence, RI, 1968.

[14] H. Li, Y. Wang and Z. Xin, Non-existence of classical solutions with finite energy to the Cauchy problem of the compressible Navier-Stokes equations, submitted, arXiv:1706.01808, 2017.

[15] J. Li and Z. Xin, Global existence of weak solutions to the barotropic compressible Navier-Stokes flows with degenerate viscosities, submitted, arXiv:1504.06826, 2016.

[16] Tatsien Li and T. Qin, Physics and Partial Differential Equations, Siam: Philadelphia, Higher Education Press: Beijing, 2014.

[17] Y. Li, R. Pan and S. Zhu, Recent progress on classical solutions for isentropic compressible NavierStokes equations with degenerate viscosities and vacuum, Bulletin of the Brazilian Mathematical Society, New Series 47 (2016), 507-519

[18] Y. Li, R. Pan and S. Zhu, On classical solutions to 2D Shallow water equations with degenerate viscosities, J. Math. Fluid Mech. 19 (2017), 151-190; arXiv:1407.8471v2, 2014.

[19] Y. Li, R. Pan and S. Zhu, On classical solutions for viscous polytropic fluids with degenerate viscosities and vacuum, submitted, arXiv: 1503.05644, 2016.

[20] T. Liu, Z. Xin and T. Yang, Vacuum states for compressible flow, Discrete Contin. Dynam. Systems 4 (1998), 1-32.

[21] A. Majda, Compressible fluid flow and systems of conservation laws in several space variables, Applied Mathematical Science 53 Spinger-Verlag: New York, Berlin Heidelberg, 1986.

[22] A. Matsumura and T. Nishida, The initial value problem for the equations of motion of viscous and heat-conductive gases. J. Math. Kyoto Univ. 20 (1980), 67-104. 
[23] A. Mellet and A. Vasseur, On the barotropic compressible Navier-Stokes equations, Commun. Part. Differ. Equations 32 (2007), 431-452.

[24] J. Nash, Le probleme de Cauchy pour les équations différentielles dún fluide général, Bull. Soc. Math. France 90 (1962), 487-491.

[25] J. Serrin, On the uniqueness of compressible fluid motion, Arch. Rational. Mech. Anal. 3 (1959), 271-288.

[26] J. Simon, Compact sets in $L^{P}(0, T ; B)$, Ann. Mat. Pura. Appl. 146 (1987), 65-96.

[27] E. Stein, Singular integrals and Differentiablility properties of Functions, Princeton Univ. Press, Princeton NJ, 1970.

[28] Z. Xin, Blow-up of smooth solutions to the compressible Navier-Stokes Equation with Compact Density, Commun. Pure. App. Math. 51 (1998), 0229-0240.

[29] Z. Xin and S. Zhu, Global well-posedness of regular solutions to the three-dimensional isentropic compressible Navier-Stokes Equations with degenerate viscosities and vacuum, submitted, arXiv: 1806.02383, 2018.

[30] Z. Xin and S. Zhu, Remarks on the existence of regular solutions with finite energy to the threedimensional isentropic compressible Navier-Stokes Equations, prepare.

[31] T. Yang and H. Zhao, A vacuum problem for the one-dimensional compressible Navier-Stokes equations with density-dependent viscisity, J. Differential Equations 184 (2002), 163-184.

[32] S. Zhu, Well-posedness and singularity formation of isentropic compressible Navier-Stokes equation, PH.D Thesis, Shanghai Jiao Tong University, 2015.

(Z.P. Xin) The Institute of Mathematical Sciences, The Chinese University of Hong Kong, Shatin, N.T., Hong Kong.

E-mail address: zpxin@ims.cuhk.edu.hk

(S. G. Zhu) Mathematical Institute, University of Oxford, Oxford OX2 6GG, UK; School of Mathematical Sciences, Monash University, Clayton, 3800, Australia; The Institute of Mathematical Sciences, The Chinese University of Hong Kong, Shatin, N.T., Hong Kong.

E-mail address: shengguo.zhu@maths.ox.ac.uk 